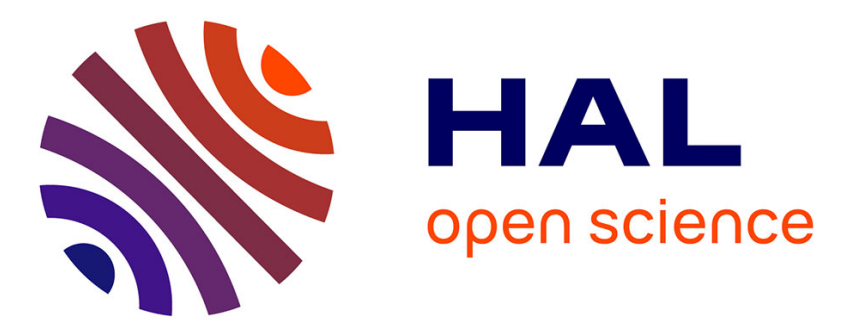

\title{
When bigger is better: intermolecular hydrofunctionalizations of activated alkenes catalyzed by heteroleptic alkaline earth complexes.
}

Bo Liu, Thierry Roisnel, Jean-François Carpentier, Yann Sarazin

\section{- To cite this version:}

Bo Liu, Thierry Roisnel, Jean-François Carpentier, Yann Sarazin. When bigger is better: intermolecular hydrofunctionalizations of activated alkenes catalyzed by heteroleptic alkaline earth complexes.. Angewandte Chemie International Edition, 2012, 51 (20), pp.4943-6. 10.1002/anie.201200364 . hal00805321

\author{
HAL Id: hal-00805321 \\ https://hal.science/hal-00805321
}

Submitted on 15 Dec 2021

HAL is a multi-disciplinary open access archive for the deposit and dissemination of scientific research documents, whether they are published or not. The documents may come from teaching and research institutions in France or abroad, or from public or private research centers.
L'archive ouverte pluridisciplinaire HAL, est destinée au dépôt et à la diffusion de documents scientifiques de niveau recherche, publiés ou non, émanant des établissements d'enseignement et de recherche français ou étrangers, des laboratoires publics ou privés. 


\title{
When Bigger is Better: Intermolecular Hydrofunctionalizations of Activated Alkenes Catalyzed by Heteroleptic Alkaline-Earth Complexes**
}

\author{
Bo Liu, Thierry Roisnel, Jean-François Carpentier* and Yann Sarazin*
}

\section{Dedicated to Dr. Christian Bruneau and Dr. Hubert Le Bozec on the occasion of their $60^{\text {th }}$ birthdays}

Catalyzed hydrofunctionalizations of unsaturated substrates are of tremendous interest, notably because of their atom efficiency. ${ }^{[1]}$ Intramolecular catalytic cyclohydroamination of aminoalkenes can be efficiently promoted by $d^{0}$ complexes of the heavy alkaline-earth (Ae) metals, ${ }^{[2-3]}$ typically $\mathrm{Ca}$ and in rare cases $\mathrm{Sr}$, as recently exemplified by the groups of Hill, ${ }^{[4]}$ Ward $^{[5]}$ and Roesky. ${ }^{[6]}$ The catalytic activity of these complexes based on large, electropositive elements (ionic radii: $\mathrm{Ca}^{2+}(6), 1.00 \AA$; $\left.\mathrm{Sr}^{2+}(6), 1.18 \AA\right)^{[7]}$ compares well with that of isoelectronic trivalent rare-earth catalysts. ${ }^{[8]}$ Through their seminal studies, Hill and coworkers have shown that the stable $\beta$-diketiminate compound $\left\{\mathrm{L}^{3}\right\} \mathrm{CaN}\left(\mathrm{SiMe}_{3}\right)_{2}(\mathrm{THF})^{[9,10,11]}$ $\left(\left\{\mathrm{L}^{3}\right\} \mathrm{H}=\mathrm{H}_{2} \mathrm{C}\left\{\mathrm{C}(\mathrm{Me}) \mathrm{N}-2,6-(i \operatorname{Pr})_{2} \mathrm{C}_{6} \mathrm{H}_{3}\right\}_{2}\right)$ is highly versatile and effective not only for intramolecular hydroamination, ${ }^{[4]}$ but also for other reactions ${ }^{[2,12]}$ such as the intermolecular hydrophosphination of alkynes and activated alkenes, ${ }^{[12 \mathrm{a}]}$ a transformation which cannot be catalyzed by trivalent rare-earth complexes thus far. ${ }^{[8 a, 13]}$

Few examples of intermolecular hydroamination reactions catalyzed by Ae complexes are known, and they involve activated alkenes, i.e. vinyl arenes and conjugated dienes. Very recently, Hultzsch reported an heteroleptic chiral magnesium-phenolate complex displaying outstanding performance for the enantioselective intra- and intermolecular hydroaminations of terminal aminoalkenes and styrene derivatives, respectively. ${ }^{[3 \mathrm{~d}]}$ Prior to this, Hill had employed homoleptic precursors $\left\{\mathrm{M}\left[\mathrm{N}\left(\mathrm{SiMe}_{3}\right)_{2}\right]_{2}\right\}_{2}$ $(\mathrm{M}=\mathrm{Ca}, \mathrm{Sr})$ to illustrate theoretical calculations on related, yet heteroleptic, systems. ${ }^{[14]}$ In the original study, the authors showed that the activity of Ae catalysts $(\mathrm{M}=\mathrm{Mg}, \mathrm{Ca}, \mathrm{Sr}, \mathrm{Ba})$ does not increase linearly with the size of the metal $\left(\mathrm{Mg}^{2+}(6), 0.72 \AA ; \mathrm{Ba}^{2+}(6)\right.$, $1.35 \AA$ ). Calculations showed that a model $\mathrm{Sr}$ heteroleptic complex should be more active in the amination of ethylene with ammonia than its $\mathrm{Ca}$ derivative (which in turn should be far more active than the $\mathrm{Mg}$ analogue), but they also suggested that the trend should not be respected with Ba. Experimental data obtained in the thorough

[*] Dr B. Liu, Dr T. Roisnel, Prof. Dr. J.-F. Carpentier, Dr Y. Sarazin

Institut des Sciences Chimiques de Rennes UMR 6226 CNRS - Université de Rennes 1 Campus de Beaulieu, 35042 Rennes Cedex (France) Fax: (+33) (0)223 236939

E-mail: yann.sarazin@univ-rennes1.fr; jean-francois.carpentier@univ-rennes1.fr Homepage: http://scienceschimiques.univrennes 1. fr/catalyse/carpentier/index.html

[**] Financial support by the European Research Council (grant FP7-People-2010-IIF, ChemCatSusDe) is gratefully acknowledged.

Supporting information for this article is available on the WWW under http://www.angewandte.org or from the author. study of the hydroamination of activated alkenes catalyzed by homoleptic complexes $\left\{\mathrm{Ae}\left[\mathrm{N}\left(\mathrm{SiMe}_{3}\right)_{2}\right]_{2}\right\}_{2}(\mathrm{Ae}=\mathrm{Mg}, \mathrm{Ca}, \mathrm{Sr}, \mathrm{Ba})$ and $\left\{\mathrm{Ae}\left[\mathrm{CH}\left(\mathrm{SiMe}_{3}\right)_{2}\right]_{2}(\mathrm{THF})_{2}\right\}(\mathrm{Ae}=\mathrm{Ca}, \mathrm{Sr})$ demonstrated that the $\mathrm{Sr}$ complex was indeed superior to the $\mathrm{Ca}$ one, while the $\mathrm{Mg}$ and $\mathrm{Ba}$ derivatives displayed very poor activities. ${ }^{[14,15]}$ Unfortunately, no experimental data were available for the series of heteroleptic complexes $\left\{\mathrm{L}^{3}\right\} \mathrm{AeN}\left(\mathrm{SiMe}_{3}\right)_{2}(\mathrm{THF})$, as the $\mathrm{Sr}$ and $\mathrm{Ba}$ species are not stable in solution. ${ }^{[10 \mathrm{~b}]}$

As part of our ongoing program aimed at implementing Aebased catalysts for a diversity of transformations, ${ }^{[16]}$ we report here the use of three families of heteroleptic complexes of the large Ae metals supported by various ancillary ligands for the antiMarkovnikov intermolecular hydroamination of vinyl arenes and isoprene. In all cases, the activity trend varies according to $(\mathrm{Mg}<)$ $\mathrm{Ca}<\mathrm{Sr}<\mathrm{Ba}$, that is, the activity increases linearly with the size of the metal. Also, the catalytic activity in the intermolecular hydrophosphination of styrene follows the same order. The Ba complexes are not only the most active in these series, but also represent the first examples of complexes of this metal capable of promoting the intermolecular hydrofunctionalizations of alkenes.

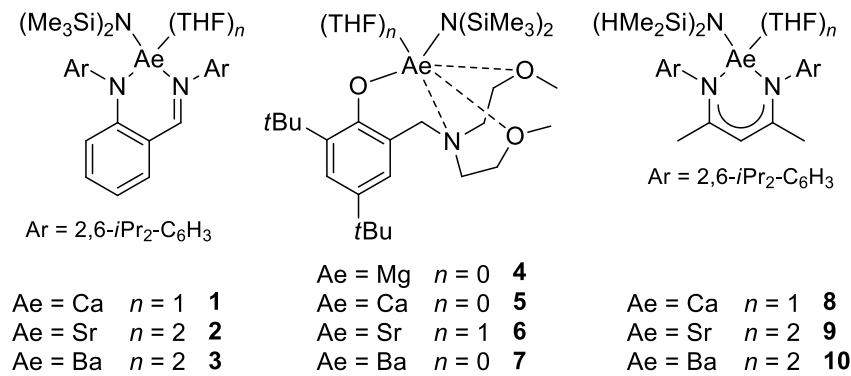

The new heteroleptic complexes $\left\{\mathrm{L}^{1}\right\} \mathrm{AeN}\left(\mathrm{SiMe}_{3}\right)_{2}(\mathrm{THF})_{n}(\mathrm{Ae}=$ Ca, $n=1,1 ; \mathrm{Sr}, n=2,2 ; \mathrm{Ba}, n=2,3)$, supported by the congested imino-anilide ligand $\left\{\mathrm{L}^{1}\right\}^{-},{ }^{[17]}$ were isolated in $45-70 \%$ yields by the one-pot reaction of $\left\{\mathrm{L}^{1}\right\} \mathrm{H}, \mathrm{AeI}_{2}$ and 2 equiv of $\mathrm{KN}\left(\mathrm{SiMe}_{3}\right)_{2} \cdot{ }^{\left[{ }^{[9]}\right.}$ The final products showed no sign of contamination by homoleptic compounds and proved stable in $\mathrm{C}_{6} \mathrm{D}_{6}$ solutions up to $60{ }^{\circ} \mathrm{C}$, as no evidence for ligand redistribution reactions could be detected. The molecular structure of the 5-coordinated complex 3 was obtained and is depicted in Figure 1; the Ba atom is located $1.14 \AA$ above the mean plane formed by the NCCCN core, and accordingly the bite angle $\mathrm{N}(1)-\mathrm{Ba}(1)-\mathrm{N}(9)$ is very narrow $\left(65.99^{\circ}\right)$. The new, stable complexes $\left\{\mathrm{L}^{2}\right\} \mathrm{AeN}\left(\mathrm{SiMe}_{3}\right)_{2}(\mathrm{THF})_{n}(\mathrm{Ae}=\mathrm{Mg}, n=0, \mathbf{4} ; \mathrm{Sr}, n=1$, 6), incorporating the tetradentate amino-ether phenolate ligand $\left\{\mathrm{L}^{2}\right\}^{-},{ }^{[18]}$ were synthesized following procedures already developed to obtain their $\mathrm{Ca}(n=0, \mathbf{5})^{[16 \mathrm{~d}]}$ and $\mathrm{Ba}(n=0,7)^{[16 \mathrm{e}]}$ analogues. Inspired by Anwander's pioneering work with rare-earth metals, ${ }^{[19]}$ we have recently shown that internal $\mathrm{Ae} \cdots \mathrm{H}-\mathrm{Si}$ agostic interactions help stabilizing heteroleptic Ae complexes against Schlenk-type equilibria. ${ }^{[16 c-f]}$ Exploiting this strategy relying on the use of the 
$\mathrm{N}\left(\mathrm{SiMe}_{2} \mathrm{H}\right)_{2}{ }^{-}$amido group, we have now prepared cleanly and in good yields $(74-78 \%)$ the complexes $\left\{\mathrm{L}^{3}\right\} \mathrm{AeN}\left(\mathrm{SiMe}_{2} \mathrm{H}\right)_{2}(\mathrm{THF})_{n}$ $(\mathrm{Ae}=\mathrm{Ca}, n=1,8 ; \mathrm{Sr}, n=2, \mathbf{9} ; \mathrm{Ba}, n=2,10)$ bearing the ubiquitous $\beta$-diketiminate ligand $\left\{\mathrm{L}^{3}\right\}^{-}$. Until now, available synthetic heteroleptic Ae precursors containing this ancillary ligand were confined to $\left\{\mathrm{L}^{3}\right\} \mathrm{MgN}\left(\mathrm{SiMe}_{3}\right)_{2}$ (and its alkyl/alkoxide derivatives $)^{[20]}$ and $\left\{\mathrm{L}^{3}\right\} \mathrm{CaN}\left(\mathrm{SiMe}_{3}\right)_{2}(\mathrm{THF}) ;^{[9,10 \mathrm{~b}, 11]}$ the $\mathrm{Sr}$ and $\mathrm{Ba}$ congeners could be prepared but were prone to ligand scrambling, which hampered the synthesis of pure compounds. ${ }^{[10 b]}$

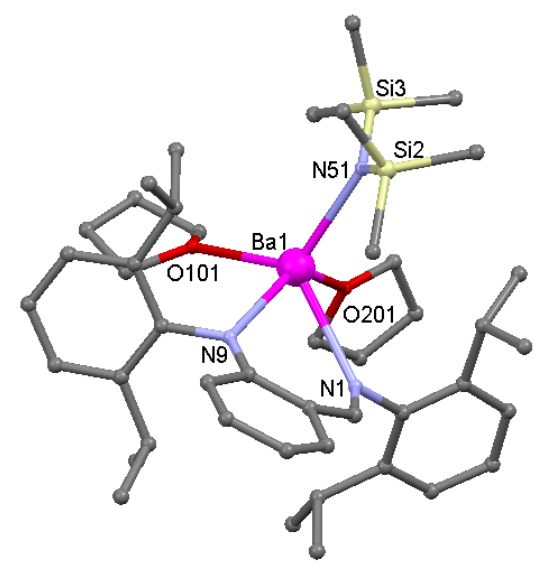

Figure 1. Representation of the molecular solid-state structure of $\left\{\mathrm{L}^{1}\right\} \mathrm{BaN}\left(\mathrm{SiMe}_{3}\right)_{2}(\mathrm{THF})_{2}(\mathbf{3})$. Only the main sites are drawn for the disordered THF molecule $(\mathrm{O}(101))$. Hydrogen atoms omitted for clarity. Selected bond lengths $[\AA]$ ] and angles [ ${ }^{\circ}$ : $\mathrm{Ba}(1)-$ $\mathrm{N}(51)$ 2.623(3), $\mathrm{Ba}(1)-\mathrm{N}(9)$ 2.677(2), $\mathrm{Ba}(1)-\mathrm{O}(201)$ 2.766(2), Ba(1)$\mathrm{O}(101)$ 2.810(3), $\mathrm{Ba}(1)-\mathrm{N}(1)$ 2.825(3), Ba(1)-Si(1) 3.676(1), Ba(1)$\mathrm{Si}(2) 3.758(1) ; \mathrm{Ba}(1)-\mathrm{N}(51)-\mathrm{Si}(2)$ 119.78(14), $\mathrm{Ba}(1)-\mathrm{N}(51)-\mathrm{Si}(3)$ 114.85(15), N(1)-Ba(1)-N(9) 65.99(8).

The ability of the new heteroleptic complexes $\mathbf{1}-\mathbf{3}$ to catalyze the intermolecular hydrofunctionalization of activated alkenes was interrogated (Table 1). A moderate catalyst loading of $2 \mathrm{~mol}-\%$ in neat substrates was typically used at $60{ }^{\circ} \mathrm{C}$. Much to our delight, 1-3 promoted the hydroamination of styrene with benzylamine, but contrary to expectations, ${ }^{[14,15]}$ we found that the performance improved substantially and regularly from $\mathrm{Ca}$ to $\mathrm{Ba}$. Where the $\mathrm{Ba}$ complex 3 achieved near-complete conversion over 18.5 h (entry 4), the $\mathrm{Ca}(\mathbf{1})$ and $\mathrm{Sr}(2)$ complexes converted respectively $34 \%$ and $71 \%$ of the substrates (entries 1 and 2). Intrigued by this phenomenon, the families of complexes 4-7 (entries 4-7) and 8-10 (entries 8-10) were also tested under rigorously identical conditions: in all cases, the trend $(\mathrm{Mg} \ll) \mathrm{Ca}<\mathrm{Sr}<\mathrm{Ba}$ was obtained, that is, irrelevantly of the identity of the ligand, the catalytic activity increased with the size of the metal. In agreement with the proposed theoretical models ${ }^{[14]}$ and experimental observations, ${ }^{[3 \mathrm{~d}, 14,15]}$ the reaction was fully regioselective, as the anti-Markovnikov product of addition to the alkene was always exclusively formed. Note that for any given metal, maximal activity was achieved with the ligand $\left\{\mathrm{L}^{3}\right\}^{-}$, whereas the lowest conversions were recorded with the phenolate $\left\{\mathrm{L}^{2}\right\}^{-}$(compare entries 1, 6, and 9;2, 7 and 10; 4, 8 and 11). This confirmed the superiority of the $\beta$-diketiminate over other ligand frameworks often observed for a number of reactions catalyzed by divalent metals. However, complexes 1-3 are more readily synthesized than $\mathbf{8}-\mathbf{1 0}$ and displayed only slightly lower efficiency. Thus, the most active catalyst in this family, the $\mathrm{Ba}$ derivative $\mathbf{3}$, was selected for subsequent investigations. The role of the identity of the amide moiety in the catalyzed reaction was negligible, as control experiments demonstrated that the activities of
$\left\{\mathrm{L}^{3}\right\} \mathrm{CaN}\left(\mathrm{SiMe}_{3}\right)_{2}(\mathrm{THF})$ and $\left\{\mathrm{L}^{2}\right\} \mathrm{BaN}\left(\mathrm{SiMe}_{2} \mathrm{H}\right)_{2}{ }^{[16 \mathrm{e}]}$ matched those of $\mathbf{8}$ and $\mathbf{7}$ respectively. ${ }^{[21]}$

The presence of an electron-donating substituent group on the aromatic ring in vinyl arenes led to a marked decrease in catalyst activity (entries 4, 19 and 20); this is consistent with earlier results with $A \mathrm{e}^{[\mathrm{3d}, 14]}$ and rare-earth metals. ${ }^{[8]}$ In our case, the presence of a chlorine atom did not lead to improved activity either (entry 21). The hydroamination of styrene with $n$-hexylamine also occurred fairly rapidly (entry 13), but the reaction was obviously sensitive to steric factors (entry 12).

Table 1. Ae-catalyzed intermolecular hydroamination of activated alkenes with amines. ${ }^{[a]}$

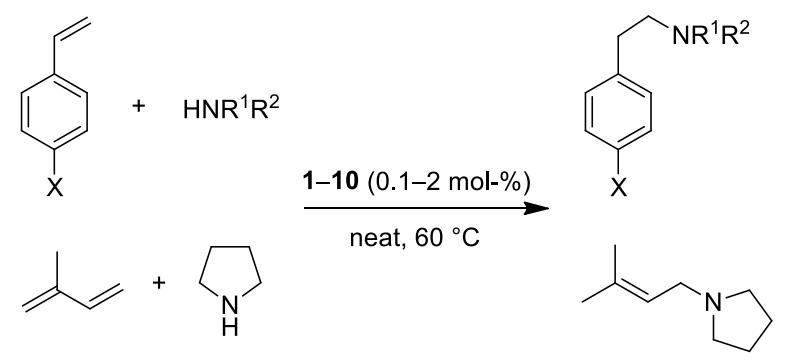

\begin{tabular}{|c|c|c|c|c|c|}
\hline Entry & Catalyst & $x$ & Amine & $\mathrm{t}[\mathrm{h}]$ & $\begin{array}{l}\text { Conv. } \\
{[\%]^{[b)}}\end{array}$ \\
\hline 1 & 1 & $\mathrm{H}$ & $\mathrm{BnNH}_{2}$ & 18.5 & 34 \\
\hline 2 & 2 & $\mathrm{H}$ & $\mathrm{BnNH}_{2}$ & 18.5 & 71 \\
\hline 3 & 3 & $\mathrm{H}$ & $\mathrm{BnNH}_{2}$ & 2 & 42 \\
\hline 4 & 3 & $\mathrm{H}$ & $\mathrm{BnNH}_{2}$ & 18.5 & 86 \\
\hline 5 & 4 & $\mathrm{H}$ & $\mathrm{BnNH}_{2}$ & 18.5 & 1 \\
\hline 6 & 5 & $\mathrm{H}$ & $\mathrm{BnNH}_{2}$ & 18.5 & 6 \\
\hline 7 & 6 & $\mathrm{H}$ & $\mathrm{BnNH}_{2}$ & 18.5 & 24 \\
\hline 8 & 7 & $\mathrm{H}$ & $\mathrm{BnNH}_{2}$ & 18.5 & 37 \\
\hline 9 & 8 & $\mathrm{H}$ & $\mathrm{BnNH}_{2}$ & 2 & 29 \\
\hline 10 & 9 & $\mathrm{H}$ & $\mathrm{BnNH}_{2}$ & 2 & 42 \\
\hline 11 & 10 & $\mathrm{H}$ & $\mathrm{BnNH}_{2}$ & 2 & 64 \\
\hline 12 & 3 & $\mathrm{H}$ & $\mathrm{Pr}_{2} \mathrm{NH}$ & 18.5 & 0 \\
\hline 13 & 3 & $\mathrm{H}$ & $n \mathrm{HexNH}_{2}$ & 18.5 & 55 \\
\hline 14 & 3 & $\mathrm{H}$ & $\left(\mathrm{CH}_{2}\right)_{4} \mathrm{NH}$ & 1 & 99 \\
\hline $15^{[c]}$ & 3 & $\mathrm{H}$ & $\left(\mathrm{CH}_{2}\right)_{4} \mathrm{NH}$ & 2 & 85 \\
\hline $16^{[c]}$ & $\mathrm{Ca}\left[\mathrm{N}\left(\mathrm{SiMe}_{3}\right)_{2}\right]_{2}(\mathrm{THF})_{2}$ & $\mathrm{H}$ & $\left(\mathrm{CH}_{2}\right)_{4} \mathrm{NH}$ & 2 & $<1$ \\
\hline $17^{[c]}$ & $\mathrm{Sr}\left[\mathrm{N}\left(\mathrm{SiMe}_{3}\right)_{2}\right]_{2}(\mathrm{THF})_{2}$ & $\mathrm{H}$ & $\left(\mathrm{CH}_{2}\right)_{4} \mathrm{NH}$ & 2 & 10 \\
\hline $18^{[\mathrm{d}]}$ & 3 & $\mathrm{H}$ & $\left(\mathrm{CH}_{2}\right)_{4} \mathrm{NH}$ & 2 & 58 \\
\hline 19 & 3 & $\mathrm{Me}$ & $\mathrm{BnNH}_{2}$ & 18.5 & 41 \\
\hline 20 & 3 & OMe & $\mathrm{BnNH}_{2}$ & 18.5 & 11 \\
\hline 21 & 3 & $\mathrm{Cl}$ & $\mathrm{BnNH}_{2}$ & 18.5 & 65 \\
\hline $22^{[\mathrm{e}]}$ & 3 & isoprene & $\left(\mathrm{CH}_{2}\right)_{4} \mathrm{NH}$ & 1 & $99^{[t]}$ \\
\hline $23^{[g]}$ & 3 & isoprene & $\left(\mathrm{CH}_{2}\right)_{4} \mathrm{NH}$ & 2 & $59^{[f]}$ \\
\hline
\end{tabular}

[a] Reaction conditions: [alkene]/[amine]/[catalyst] $=50: 50: 1$ unless otherwise specified, $10.5 \mu \mathrm{mol}$ of catalyst, no additional solvent, $\mathrm{T}=$ $60^{\circ} \mathrm{C}$. [b] Determined by ${ }^{1} \mathrm{H}$ NMR spectroscopy. [c]

[styrene]/[pyrrolidine]/[3] = 500:500:1. [d] [styrene]/[pyrrolidine]/[3] = 1000:1000:1. [e] [isoprene]/[pyrrolidine]/[3] = 220:50:1. [f] Based on amine conversion. [g] [isoprene]/[pyrrolidine]/[3] = 2000:1000:1.

The fastest reaction rates were achieved with pyrrolidine, as conversion of 50 equiv was complete within $1 \mathrm{~h}$ (entry 14). With [styrene]/[pyrrolidine]/[3] = 500:500:1 (entry 15) or 1000:1000:1 (entry 18 ), $85 \%$ and $58 \%$ conversion were achieved in $2 \mathrm{~h}$, with corresponding turnover frequencies of 212 and $290 \mathrm{~h}^{-1}$. These values, achieved under mild conditions, exceed by 1 to 2 orders of 
magnitude those reported to date for intermolecular hydroamination reactions catalyzed by Ae, ${ }^{[3 \mathrm{~d}, 14,15]}$, rare-earth ${ }^{[8]}$ or even late-transition metal $^{[22]}$ complexes; note that under identical conditions, the best ${ }^{[15]}$ bis(amides) $\mathrm{Ae}\left[\mathrm{N}\left(\mathrm{SiMe}_{3}\right)_{2}\right]_{2}(\mathrm{THF})_{2}(\mathrm{Ae}=\mathrm{Ca}$, entry $16, \mathrm{Sr}$, entry 17$)$, and homoleptic complexes recently reported ${ }^{[23]}$ displayed vastly lower reaction rates. The selection of alkene was not restricted to vinyl arenes, as $\mathbf{3}$ catalyzed the reaction of isoprene and pyrrolidine with equal competence (entries 22-23). Full conversion was observed within $1 \mathrm{~h}$ with 2 mol- $\%$ of $\mathbf{3}$, and gratifyingly the conversion reached $59 \%$ after $2 \mathrm{~h}\left(\mathrm{TOF}=295 \mathrm{~h}^{-1}\right)$ when as little as 0.1 mol- $\%$ of 3 was employed. The reaction was 1,4-regioselective, with anti-Markovnikov addition of pyrrolidine occurring exclusively on the least encumbered unsaturation to give 1-(3-methylbut-2-en-1yl)pyrrolidine.

Kinetic studies of the hydroamination of styrene with pyrrolidine catalyzed by $\mathbf{3}$ were performed under a broad range of amine, styrene and catalyst concentrations by ${ }^{1} \mathrm{H} \quad \mathrm{NMR}$ monitoring. ${ }^{[21]}$ The determined empirical rate-law (1),

$$
v=k[\text { styrene }]^{1.0}[\text { pyrrolidine }]^{1.0}[3]^{1.0}
$$

with partial first-order in each of the components of the system, differs from that established for rare-earth catalysts: Marks showed that the kinetics of the hydroamination of alkynes with primary amines were zero-order in amine and first-order in catalyst and alkyne concentrations. ${ }^{[8,24]}$ The activation parameters for $\mathbf{3}, E_{\mathrm{a}}=$ 19.0(0.6) $\mathrm{kcal} \cdot \mathrm{mol}^{-1}, \Delta \mathrm{H}^{\ddagger}=18.3(0.8) \mathrm{kcal} \cdot \mathrm{mol}^{-1}$ and $\Delta \mathrm{S}^{\ddagger}=-$ 13.1(2.7) $\mathrm{cal} \cdot \mathrm{mol}^{-1} \cdot \mathrm{K}^{-1}$, were extracted by Arrhenius and Eyring analyses of kinetic data obtained in the temperature range $25-60{ }^{\circ} \mathrm{C}$. These values are diagnostic of ordered transition states. The calculated $\Delta \mathrm{G}^{\ddagger}$ for this process at $298 \mathrm{~K}$ is $22.4 \mathrm{kcal} \cdot \mathrm{mol}^{-1}$, which compares favorably with the values reported for $\left\{\mathrm{Ca}\left[\mathrm{N}\left(\mathrm{SiMe}_{3}\right)_{2}\right]_{2}\right\}_{2}$ $\left(24.1 \mathrm{kcal} \cdot \mathrm{mol}^{-1}\right)$ and $\left\{\mathrm{Sr}\left[\mathrm{N}\left(\mathrm{SiMe}_{3}\right)_{2}\right]_{2}\right\}_{2}\left(23.4 \mathrm{kcal} \cdot \mathrm{mol}^{-1}\right)$ for the catalyzed hydroamination of styrene with piperidine. ${ }^{[14,15]}$

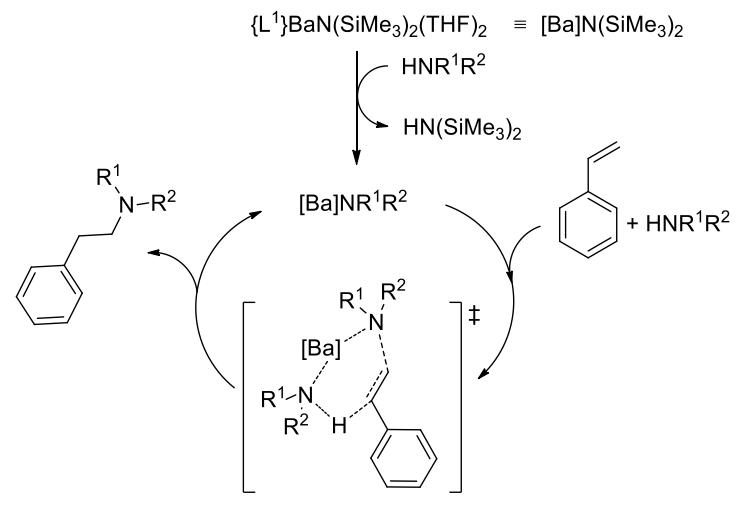

Scheme 1. Possible six-centered concerted mechanistic pathway for styrene/amine intermolecular hydroamination catalyzed by 3 .

The rate-law featured in the case of $\mathbf{3}$ points out at a different mechanism from that proposed for rare-earth systems, or at least one where the rate-limiting step is not the intermolecular insertion of the alkene into the $\mathrm{Ba}-\mathrm{N}$ (pyrrolidine) bond. A plausible mechanism compatible with equation (1) involves a one-step, non-insertive route with a six-centered transition state (Scheme 1). It proceeds via concerted proton transfer onto the unsaturation activated towards the attack of the nucleophile. This hypothesis, where the $\mathrm{N}-\mathrm{H}$ bond plays a key role in the transition state, is corroborated by the strong kinetic isotope effect (KIE) observed during the monitoring of the reaction of styrene with deuterated pyrrolidine catalyzed by $3: k_{\mathrm{H}} / k_{\mathrm{D}}$ ratios of 6.8 and 7.3 were found at $40{ }^{\circ} \mathrm{C}$ and $60{ }^{\circ} \mathrm{C}$ respectively. ${ }^{[21]}$ These values are intermediary between the theoretical maximum of $8.5^{[25]}$ or the ratio reported for $\left\{\mathrm{Sr}\left[\mathrm{N}\left(\mathrm{SiMe}_{3}\right)_{2}\right]_{2}\right\}_{2}\left(k_{\mathrm{H}} / k_{\mathrm{D}}=7.9\right.$ at $\left.55^{\circ} \mathrm{C}\right)$, and the values found for $\left\{\mathrm{Ca}\left[\mathrm{N}\left(\mathrm{SiMe}_{3}\right)_{2}\right]_{2}\right\}_{2}\left(k_{\mathrm{H}} / k_{\mathrm{D}}=4.3\right.$ and 4.1 at $70{ }^{\circ} \mathrm{C}$ and $55{ }^{\circ} \mathrm{C}$ respectively $)^{[15]}$ or $\left(\mathrm{C}_{5} \mathrm{Me}_{5}\right)_{2} \mathrm{LaCH}\left(\mathrm{SiMe}_{3}\right)_{2}$ $\left(k_{\mathrm{H}} / k_{\mathrm{D}}=4.1\right.$ at $\left.25^{\circ} \mathrm{C}\right) .{ }^{[26]}$ Note that a related mechanism has been debated for the intramolecular cyclohydroamination of aminoalkenes with a Mg complex, ${ }^{[3 \mathrm{~b}, \mathrm{c}]}$ while that reported during submission of this manuscript by Hill for intermolecular reactions catalyzed by homoleptic complexes also bears strong analogy. ${ }^{[15]}$

Complexes 1-3, 5-7 and 8-10 also all catalyzed the intermolecular hydrophosphination of styrene with $\mathrm{HPCy}_{2}$ or $\mathrm{HPPh}_{2}$ (catalyst loading $2 \mathrm{~mol}-\%, 60{ }^{\circ} \mathrm{C}$, neat). Here also, the activity trend was $\mathrm{Ca}<\mathrm{Sr}<\mathrm{Ba}$, although the performances now increased according to $\left\{\mathrm{L}^{3}\right\}^{-}<\left\{\mathrm{L}^{1}\right\}^{-} \approx\left\{\mathrm{L}^{2}\right\}^{-}$. $^{[21]}$ The reactions proceeded with perfect anti-Markovnikov regioselectivity. With $\mathrm{HPCy}_{2}$, partial conversion only was achieved after $18.5 \mathrm{~h}$, even with $\mathbf{1}-\mathbf{3}$, the most active complexes for this transformation ( $c a .42 \%$ with 3 ). On the other hand, the reactions with the less basic $\mathrm{HPPh}_{2}$ were considerably faster, and $96 \%$ conversion was achieved using $\mathbf{3}$ in as little as $15 \mathrm{~min}$. The corresponding TOF $\left(192 \mathrm{~h}^{-1}\right)$ outclasses that reported with $\left\{\mathrm{L}^{3}\right\} \mathrm{CaN}\left(\mathrm{SiMe}_{3}\right)_{2}(\mathrm{THF})\left(\right.$ ca. $0.5 \mathrm{~h}^{-1}$ at $\left.75{ }^{\circ} \mathrm{C}\right),{ }^{[12 \mathrm{a}]}$ whereas rare-earth complexes are not known to catalyze this reaction.

In conclusion, several complete families of stable complexes of the large Ae metals which catalyze intermolecular hydrofunctionalization reactions of activated alkenes have been prepared. Contrary to expectations based on previous computations, it was found that the catalyst activity increased systematically with the size of the metal, and the barium complexes have, for the first time, displayed impressive efficacy in these catalytic reactions. In particular, the stable and readily accessed imino-anilide barium complex $\left\{\mathrm{L}^{1}\right\} \mathrm{BaN}\left(\mathrm{SiMe}_{3}\right)_{2}(\mathrm{THF})_{2} \quad$ (3) offers real potential as catalyst for a variety of organic transformations. We are now exploring this avenue while further studies are also underway to assess the validity of the proposed mechanism.

\section{Experimental Section}

Details for the syntheses and characterization of compounds 1-10 and experimental protocols for catalytic tests are given in the Supporting information.

Received: ((will be filled in by the editorial staff))

Published online on ((will be filled in by the editorial staff))

Keywords: large alkaline-earth metals · barium · intermolecular hydroamination $\cdot$ hydrophosphination $\cdot$ catalysis

[1] Catalytic Heterofunctionalization: from Hydroamination to Hydrozirconation (Eds.: A. Togni, H. Grützmacher), Wiley-VCH, Weinheim, 2001.

[2] a) S. Harder, Chem. Rev., 2010, 110, 3852-3876; b) A. G. M. Barrett, M. R. Crimmin, M. S. Hill, P. A. Procopiou, Proc. R. Soc. London Ser. A, 2010, 466, 927-963.

[3] For Mg studies, see: a) X. Zhang, T. J. Emge, K. C. Hultzsch, Organometallics, 2010, 29, 5871-5877; b) J. F. Dunne, D. Bruce Fulton, A. Ellern, A. D. Sadow, J. Am. Chem. Soc., 2010, 132, 17680- 
17683; c) S. Tobisch, Chem. - Eur. J., 2011, 17, 14974-14986; d) T. J. Emge, K. C. Hultzsch, Angew. Chem. Int. Ed., 2012, 51, 394-398.

[4] a) M. R. Crimmin, I. J. Casely, M. S. Hill, J. Am. Chem. Soc., 2005, 127, 2042-2043; b) A. G. M. Barrett, M. R. Crimmin, M. S. Hill, P. B Hitchcock, G. Kociok-Köhn, P. A. Procopiou, Inorg. Chem., 2008, 47, 7366-7376; c) A. G. M. Barrett, I. J. Casely, M. R. Crimmin, M. S. Hill, J. R. Lachs, M. F. Mahon, P. A. Procopiou, Inorg. Chem., 2009, 48, 4445-4453; d) M. R. Crimmin, M. Arrowsmith, A. G. M. Barrett, I. J. Casely, M. S. Hill, P. A. Procopiou, J. Am. Chem. Soc., 2009, 131, 9670-9685; e) M. Arrowsmith, M. S. Hill, G. Kociok-Köhn, Organometallics, 2009, 28, 1730-1738; f) M. Arrowsmith, M. S. Hill, G. Kociok-Köhn, Organometallics, 2011, 30, 1291-1294; g) M. Arrowsmith, M. R. Crimmin, A. G. M. Barrett, M. S. Hill, G. KociokKöhn, P. A. Procopiou, Organometallics, 2011, 30, 1493-1506.

[5] a) J. S. Wixey, B. D. Ward, Chem. Commun., 2011, 47, 5449-5451; b) J. S. Wixey, B. D. Ward, Dalton Trans., 2011, 40, 7693-7696.

[6] a) S. Datta, P. W. Roesky, S. Blechert, Organometallics, 2007, 26, 4392-4394; b) S. Datta, M. T. Gamer, P. W. Roesky, Organometallics, 2008, 27, 1207-1213; c) J. Jenter, R. Köppe, P. W. Roesky, Organometallics, 2011, 30, 1404-1413.

[7] R. D. Shannon, Acta Cryst., 1976, A32, 751-767.

[8] a) S. Hong, T. J. Marks, Acc. Chem. Res., 2004, 37, 673-686; b) K. C. Hultzsch, Adv. Synth. Catal., 2005, 347, 367-391; c) T. E. Müller, K. C. Hultzsch, M. Yus, F. Foubelo, M. Tada, Chem. Rev., 2008, 108, 3795-3892.

[9] a) M. H. Chisholm, J. Gallucci, K. Phomphrai, Chem. Commun., 2003, 48-49; b) ) M. H. Chisholm, J. Gallucci, K. Phomphrai, Inorg. Chem., 2004, 43, 6717-6725.

[10] a) M. S. Hill, P. B. Hitchcock, Chem. Commun., 2003, 1758-1759; b) A. G. Avent, M. R. Crimmin, M. S. Hill, P. B. Hitchcock, Dalton Trans., 2005, 278-284.

[11] a) A. G. Avent, M. R. Crimmin, M. S. Hill, P. B. Hitchcock, Dalton Trans., 2004, 3166-3168; b) A. G.M. Barrett, M. R. Crimmin, M. S. Hill, G. Kociok-Köhn, J. R. Lachs, P. A. Procopiou, Dalton Trans., 2008, 1292-1294.

[12] a) M. R. Crimmin, A. G. M. Barrett, M. S. Hill, P. B. Hitchcock, P. A. Procopiou, Organometallics, 2007, 26, 2953-2956; b) A. G. M. Barrett, T. C. Boorman, M. R. Crimmin, M. S. Hill, G. Kociok-Köhn, P. A. Procopiou, Chem. Commun., 2008, 5206-5208; c) J. R. Lachs, A G. M. Barrett, M. R. Crimmin, G. Kociok-Köhn, M. S. Hill, M. F. Mahon, P. A. Procopiou, Eur. J. Inorg. Chem., 2008, 4173-4179.
[13] Divalent organoytterbium species are known to promote the intermolecular hydrophosphination of alkynes: K. Takaki, G. Koshoji, K. Komeyama, M. Takeda, T. Shishido, A. Kitani, K. Takehira, J. Org. Chem., 2003, 68, 6554-6565.

[14] A. G. M. Barrett, C. Brinkmann, M. R. Crimmin, M. S. Hill, P. Hunt, P. A. Procopiou, J. Am. Chem. Soc., 2009, 131, 12906-12907.

[15] C. Brinkmann, A. G. M. Barrett, M. S. Hill, P. A. Procopiou, J. Am. Chem. Soc., 2012, 134, 2193-2207.

[16] a) V. Poirier, T. Roisnel, J.-F. Carpentier, Y. Sarazin, Dalton Trans., 2009, 9820-9827; b) Y. Sarazin, V. Poirier, T. Roisnel, J.-F. Carpentier, Eur. J. Inorg. Chem., 2010, 3423-3428; c) Y. Sarazin, D. Roşca, V. Poirier, T. Roisnel, A. Silvestru, L. Maron, J.-F. Carpentier, Organometallics, 2010, 29, 6569-6577; d) Y. Sarazin, B. Liu, T. Roisnel, L. Maron, J.-F. Carpentier, J. Am. Chem. Soc., 2011, 133, 9069-9087; e) B. Liu, T. Roisnel, Y. Sarazin, Inorg. Chim. Acta, 2012 380, 2-13; f) B. Liu, T. Roisnel, J.-P. Guégan, J.-F. Carpentier, Y. Sarazin, Chem. Eur. J., doi: 10.1002/chem.201103666.

[17] P. G. Hayes, G. C. Welch, D. J. H. Emslie, C. L. Noack, W. E. Piers, M. Parvez, Organometallics, 2003, 22, 1577-1579.

[18] S. Groysman, E. Sergeeva, I. Goldberg, M. Kol, Inorg. Chem., 2005 , 44, 8188-8190.

[19] a) W. A. Herrmann, J. Eppinger, M. Spiegler, O. Runte, R. Anwander, Organometallics, 1997, 16, 1813-1815; b) R. Anwander, O. Runte, J. Eppinger, G. Gerstberger, E. Herdtweck, M. Spiegler, J. Chem. Soc., Dalton Trans., 1998, 847-858; c) I. Nagl, W. Scherer, M. Tafipolsky, R. Anwander, Eur. J. Inorg. Chem., 1999, 1405-1407; d) W. Hieringer, J. Eppinger, R. Anwander, W. A. Herrmann, J. Am. Chem. Soc., 2000, 122, 11983-11994.

[20] a) M. H. Chisholm, J. C. Huffman, K. Phomphrai, J. Chem. Soc., Dalton Trans., 2001, 222-224; b) B. M. Chamberlain, M. Cheng, D. R. Moore, T. M. Ovitt, E. B. Lobkovsky, G. W. Coates, J. Am. Chem. Soc., 2001, 123 , 3229-3238.

[21] See the Supporting information for details

[22] K. D. Hesp, M. Stradiotto, ChemCatChem, 2010, 2, 1192-1207.

[23] The reaction of styrene with pyrrolidine (1:1) catalyzed by $5 \mathrm{~mol}-\%$ of $\left\{\mathrm{Sr}\left[\mathrm{N}\left(\mathrm{SiMe}_{3}\right)_{2}\right]_{2}\right\}_{2}$ reached $65 \%$ conversion after $3.5 \mathrm{~h}$, see ref. [15].

[24] J.-S. Ryu, G. Yanwu Li, T. J. Marks, J. Am. Chem. Soc., 2003, 125 , 12584-12605.

[25] R. P. Bell, The proton in chemistry ( $2^{\text {nd }}$ ed.), Cornell University Press, Ithaca, 1973.

[26] M. R. Gagné, C. L. Stern, T. J. Marks, J. Am. Chem. Soc., 1992, 114, 275-294. 
Intermolecular

hydroelementations

B. Liu, T. Roisnel, J.-F. Carpentier, Y. Sarazin Page - Page

When Bigger is Better: Intermolecular Hydrofunctionalizations of Activated Alkenes Catalyzed by Heteroleptic Alkaline-Earth Complexes

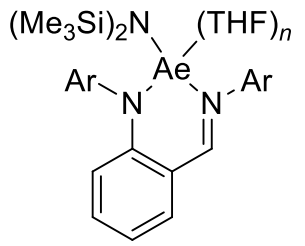

$\mathrm{Ar}=2,6-i \mathrm{Pr}_{2}-\mathrm{C}_{6} \mathrm{H}_{3}$ $\mathrm{Ae}=\mathrm{Ca}, \mathrm{Sr}, \mathrm{Ba}$
[Ae] $0.1-2 \mathrm{~mol}-\% \quad X$ neat, $60{ }^{\circ} \mathrm{C}$ $\mathrm{Ca}<\mathrm{Sr}<\mathrm{Ba}$

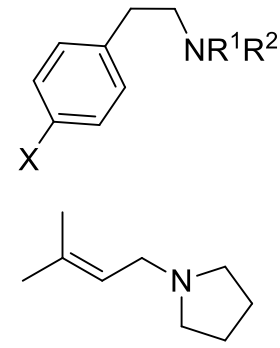

Big but fast! New amino-phenolate, imino-anilido and $\beta$-diketiminate alkaline-earth amido complexes catalyze the regioselective intermolecular hydroamination and hydrophosphination of styrene and isoprene with unprecedented activities; the catalytic performances increased linearly with the size of the metal. 


\section{Supporting Information for}

"When Bigger is Better: Intermolecular Hydrofunctionalizations of Activated Alkenes Catalyzed by Heteroleptic Alkaline-Earth Complexes"

Bo Liu, Thierry Roisnel, Jean-François Carpentier* and Yann Sarazin*

S1 General experimental procedures

S2 Synthesis and characterization of $\left\{\mathrm{L}^{1}\right\} \mathrm{CaN}\left(\mathrm{SiMe}_{3}\right)_{2}(\mathrm{THF})$ (1)

S3 Synthesis and characterization of $\left\{\mathrm{L}^{1}\right\} \mathrm{SrN}\left(\mathrm{SiMe}_{3}\right)_{2}(\mathrm{THF})_{2}(2)$

S4 Synthesis and characterization of $\left\{\mathrm{L}^{1}\right\} \mathrm{BaN}\left(\mathrm{SiMe}_{3}\right)_{2}(\mathrm{THF})_{2}(\mathbf{3})$

S5 Synthesis and characterization of $\left\{\mathrm{L}^{2}\right\} \mathrm{MgN}\left(\mathrm{SiMe}_{3}\right)_{2}(\mathbf{4})$

S6 Synthesis and characterization of $\left\{\mathrm{L}^{2}\right\} \mathrm{SrN}\left(\mathrm{SiMe}_{3}\right)_{2}$ (THF) (6)

S7 Synthesis and characterization of $\left\{\mathrm{L}^{3}\right\} \mathrm{CaN}\left(\mathrm{SiMe}_{2} \mathrm{H}\right)_{2}(\mathrm{THF})(\mathbf{8})$

S8 Synthesis and characterization of $\left\{\mathrm{L}^{3}\right\} \mathrm{SrN}\left(\mathrm{SiMe}_{2} \mathrm{H}\right)_{2}(\mathrm{THF})_{2}$ (9)

S9 Synthesis and characterization of $\left\{\mathrm{L}^{3}\right\} \mathrm{BaN}\left(\mathrm{SiMe}_{2} \mathrm{H}\right)_{2}(\mathrm{THF})_{2}(\mathbf{1 0})$

S10 Characterization of new hydroamination and hydrophosphination products

S11 Typical protocol for intermolecular hydrofunctionalization reactions

S12 X-ray structure of $\left\{\mathrm{L}^{1}\right\} \mathrm{CaN}\left(\mathrm{SiMe}_{3}\right)_{2}(\mathrm{THF})(\mathbf{1})$

$\mathrm{S} 13 \mathrm{X}$-ray structure of $\left\{\mathrm{L}^{2}\right\} \mathrm{MgN}\left(\mathrm{SiMe}_{3}\right)_{2}(\mathbf{4})$

S14 X-ray structure of $\left\{\mathrm{L}^{2}\right\} \mathrm{SrN}\left(\mathrm{SiMe}_{3}\right)_{2}(\mathrm{THF})(\mathbf{6})$

S15 X-ray structure of $\left\{\mathrm{L}^{3}\right\} \mathrm{CaN}\left(\mathrm{SiMe}_{2} \mathrm{H}\right)_{2}(\mathrm{THF})(\mathbf{8})$

S16 X-ray structure of $\left\{\mathrm{L}^{3}\right\} \mathrm{BaN}\left(\mathrm{SiMe}_{2} \mathrm{H}\right)_{2}(\mathrm{THF})_{2}(\mathbf{1 0})$

S17 Tables of crystallographic data

S18 Experimental details for the acquisition of $\mathrm{X}$-ray crystallographic data

S19 Full data table for the hydroamination of styrene with $\mathrm{BnNH}_{2}$ catalyzed by 1-10

S20 Full data table for intermolecular hydroamination reactions catalyzed by 3

S21 Full data table for intermolecular hydrophosphination reactions catalyzed by $\mathbf{3}$

S22 Plot of $\ln \left([\text { pyrrolidine }]_{0} /[\text { pyrrolidine }]_{t}\right) v s$. reaction time for the hydroamination of styrene with pyrrolidine catalyzed by 3 at different concentrations of styrene

S23 Plot of $\ln k_{\text {app }} v s . \ln [\text { styrene }]_{0}$ for the hydroamination of styrene with pyrrolidine catalyzed by 3

S24 Plot of $\ln \left([\text { pyrrolidine }]_{0} /[\text { pyrrolidine }]_{\mathrm{t}}\right) v s$. reaction time (s) for the hydroamination of styrene with pyrrolidine catalyzed by $\mathbf{3}$ at different concentrations of $\mathbf{3}$

S25 Plot of $\ln k_{\text {app }} v s . \ln [3]_{0}$ for the hydroamination of styrene with pyrrolidine catalyzed by 3

S26 Plot of $\ln$ ([pyrrolidine]//[pyrrolidine]t) vs. reaction time (s) for the hydroamination of styrene with pyrrolidine catalyzed by 3 at $333,323,313,298 \mathrm{~K}$

S27 Arrhenius plot of $\ln k_{\text {app }} v s .1 / \mathrm{T}\left(\mathrm{K}^{-1}\right)$ for the hydroamination of styrene and pyrrolidine catalyzed by 3

S28 Eyring Plot of $\ln \left(k_{\mathrm{app}} \mathrm{h} / \mathrm{k}_{\mathrm{B}} \mathrm{T}\right)$ vs. $1 / \mathrm{T}\left(\mathrm{K}^{-1}\right)$ for the hydroamination of styrene and pyrrolidine catalyzed by 3

S29 Kinetic isotope effect experiments

S30 References 


\section{S1 General experimental procedures}

All manipulations were performed under inert atmosphere using standard Schlenk techniques or in a Jacomex glove-box $\left(\mathrm{O}_{2}<1\right.$ ppm, $\mathrm{H}_{2} \mathrm{O}<5$ ppm) for catalyst loading.

NMR spectra were recorded on Bruker AC-300, AC-400 and AM-500 spectrometers. All chemicals shifts were determined using residual signals of the deuterated solvents and were calibrated vs. $\mathrm{SiMe}_{4}$. Assignment of the signals was carried out using $1 \mathrm{D}\left({ }^{1} \mathrm{H},{ }^{13} \mathrm{C}\left\{{ }^{1} \mathrm{H}\right\}\right)$ and 2D (COSY, HMBC, HMQC) NMR experiments. Coupling constants are given in Hertz.

Elemental analyses were performed on a Carlo Erba 1108 Elemental Analyser instrument at the London Metropolitan University by Stephen Boyer and were the average of a minimum of two independent measurements. $\mathrm{CaI}_{2}, \mathrm{SrI}_{2}, \mathrm{BaI}_{2}$ (anhydrous beads, 99.995\%) were purchased from Aldrich and used as received. $\mathrm{HN}\left(\mathrm{SiMe}_{3}\right)_{2}$ (Acros), $\mathrm{HN}\left(\mathrm{SiMe}_{2} \mathrm{H}\right)_{2}(\mathrm{ABCR})$ were dried over activated $3 \AA$ molecular sieves and distilled under reduced pressure prior to use. Styrene, 4-chlorostyrene, 4-methoxystyrene, 4-methylstyrene, isoprene, benzylamine, diisopropylamine, 2-methoxyethylamine, aniline, hexylamine, pyrrolidine, dicyclohexylphosphine and diphenylphosphine were purchased from Aldrich, Acros or ABCR. All were vacuum-distilled over $\mathrm{CaH}_{2}$ (except dicyclohexylphosphine and diphenylphosphine which were used without further purification), and then were degassed by freeze-pump-thaw methods. THF was first pre-dried over activated alumina (SPS MBraun system), and then freshly distilled under argon from Na/benzophenone prior to use. Pentane was distilled under argon from $\mathrm{Na}$ /benzophenone/tetraglyme. All deuterated solvents (Eurisotop, Saclay, France) were stored in sealed ampoules over activated $3 \AA$ molecular sieves and were thoroughly degassed by several freeze-thaw cycles. In a procedure adapted from the literature, ${ }^{[1]} N$-deuterated pyrrolidine (pyrrolidine- $d_{1}$ ) used for kinetic isotope effect experiments was prepared by refluxing pyrrolidine in a large excess of $\mathrm{D}_{2} \mathrm{O}$, followed by distillation under atmospheric pressure in a $15 \mathrm{~cm}$ column packed with Fenske helices.

The synthetic precursors $\mathrm{Ae}\left[\mathrm{N}\left(\mathrm{SiMe}_{2} \mathrm{H}\right)_{2}\right]_{2}(\mathrm{THF})_{n}{ }^{[2]}$ and $\mathrm{Ae}\left[\mathrm{N}\left(\mathrm{SiMe}_{3}\right)_{2}\right]_{2}(\mathrm{THF})_{2}{ }^{[3]}(\mathrm{Ae}=\mathrm{Ca}, \mathrm{Sr}, \mathrm{Ba})$, the complexes, $\left\{\mathrm{L}^{2}\right\} \mathrm{CaN}\left(\mathrm{SiMe}_{3}\right)_{2}(\mathbf{5}),{ }^{[4]}\left\{\mathrm{L}^{2}\right\} \mathrm{BaN}\left(\mathrm{SiMe}_{3}\right)_{2}(7),{ }^{[5]}\left\{\mathrm{L}^{2}\right\} \mathrm{BaN}\left(\mathrm{SiMe}_{2} \mathrm{H}\right)_{2},{ }^{[5]}$ and the pro-ligands $\left\{\mathrm{L}^{1}\right\} \mathrm{H}^{[}{ }^{[6]}\left\{\mathrm{L}^{2}\right\} \mathrm{H}^{[7]}$ and $\left\{\mathrm{L}^{3}\right\} \mathrm{H}^{[8]}$ and were all prepared as described elsewhere. 


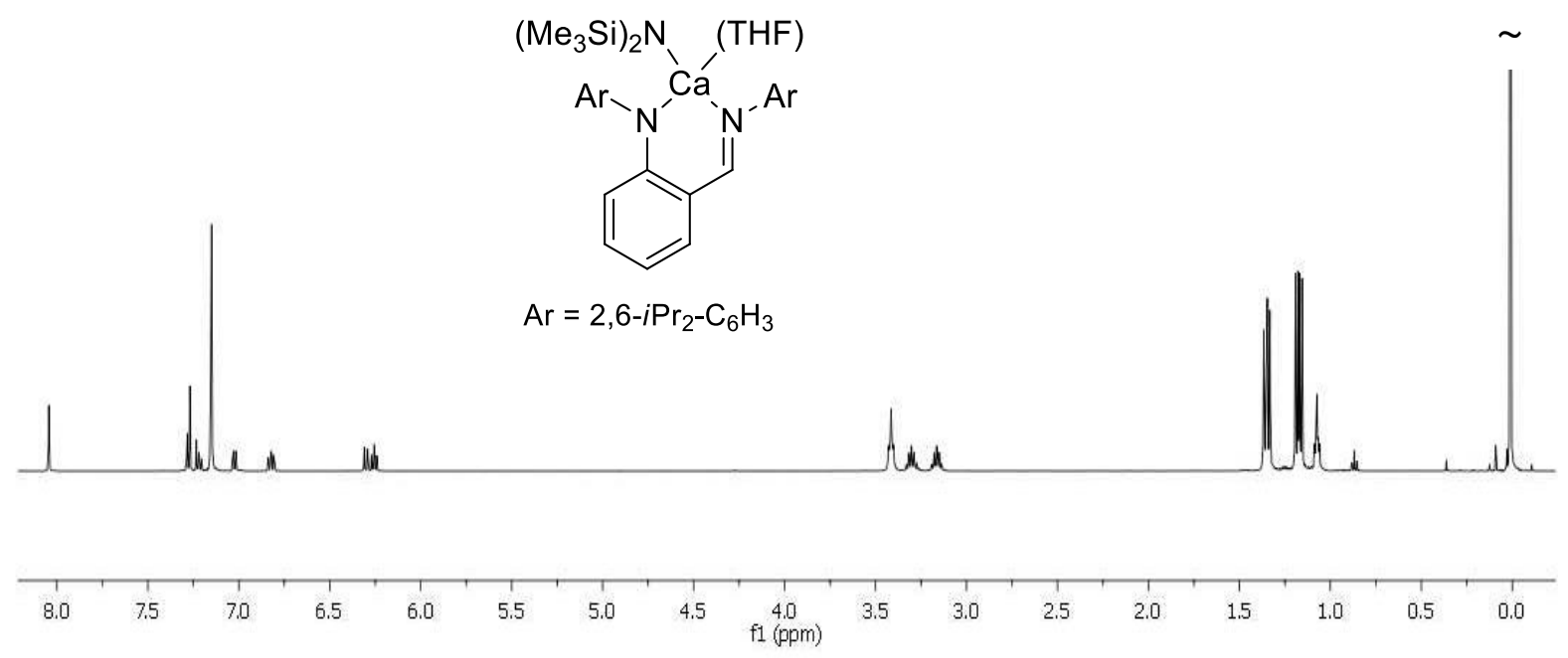

${ }^{1} \mathrm{H}$ NMR spectrum $\left(\mathrm{C}_{6} \mathrm{D}_{6}, 298 \mathrm{~K}, 500.13 \mathrm{MHz}\right)$ of $\mathbf{1}$.

$\left\{\mathrm{L}^{1}\right\} \mathrm{H}(0.60 \mathrm{~g}, 1.36 \mathrm{mmol})$ and $\mathrm{KN}\left(\mathrm{SiMe}_{3}\right)_{2}(0.54 \mathrm{~g}, 2.72 \mathrm{mmol})$ were dissolved in THF $(20 \mathrm{~mL})$. After the mixture was stirred for $1 \mathrm{~h}$, it was added to a suspension of $\mathrm{CaI}_{2}(0.42 \mathrm{~g}, 1.43 \mathrm{mmol})$ in THF $(10 \mathrm{~mL})$ and the resulting mixture was stirred for $2.5 \mathrm{~h}$. Removal of the solvent under vacuum and extraction of the residue with pentane (50 $\mathrm{mL})$ and extraction of the volatile fraction gave $1(0.58 \mathrm{~g}, 60 \%)$ as a yellow solid. Crystals suitable for singlecrystal X-ray diffraction crystallography were obtained by from concentrated pentane solution soptred overnight at $-26{ }^{\circ} \mathrm{C} .{ }^{1} \mathrm{H}$ NMR $\left(\mathrm{C}_{6} \mathrm{D}_{6}, 298 \mathrm{~K}, 500.13 \mathrm{MHz}\right): \delta=8.05(\mathrm{~s}, 1 \mathrm{H}, \mathrm{CH}=\mathrm{N}), 7.28\left(\mathrm{~d},{ }^{3} J_{\mathrm{HH}}=7.2 \mathrm{~Hz}, 2 \mathrm{H}\right.$, arom- $\left.H\right), 7.21$ $\left(\mathrm{t},{ }^{3} J_{\mathrm{HH}}=7.8 \mathrm{~Hz}, 1 \mathrm{H}\right.$, arom- $\left.H\right), 7.14(\mathrm{~m}, 3 \mathrm{H}, \operatorname{arom}-H), 7.05\left(\mathrm{dd},{ }^{3} J_{\mathrm{HH}}=7.9 \mathrm{~Hz},{ }^{4} J_{\mathrm{HH}}=1.8 \mathrm{~Hz}, 1 \mathrm{H}\right.$, arom- $\left.H\right), 6.87$ $\left(\mathrm{td},{ }^{3} J_{\mathrm{HH}}=8.0 \mathrm{~Hz},{ }^{4} J_{\mathrm{HH}}=1.8 \mathrm{~Hz}, 1 \mathrm{H}\right.$, arom $\left.-H\right), 6.26(\mathrm{~m}, 2 \mathrm{H}$, arom- $H), 3.42(\mathrm{~m}, 4 \mathrm{H}, \mathrm{THF}), 3.32(\mathrm{~m}, 2 \mathrm{H}$, $\left.\mathrm{CH}\left(\mathrm{CH}_{3}\right)_{2}\right), 3.17\left(\mathrm{~m}, 2 \mathrm{H}, \mathrm{CH}\left(\mathrm{CH}_{3}\right)_{2}\right), 1.33\left(\mathrm{~d},{ }^{3} J_{\mathrm{HH}}=6.8 \mathrm{~Hz}, 6 \mathrm{H}, \mathrm{CH}\left(\mathrm{CH}_{3}\right)_{2}\right), 1.31\left(\mathrm{~d},{ }^{3} J_{\mathrm{HH}}=6.8 \mathrm{~Hz}, 6 \mathrm{H}\right.$, $\left.\mathrm{CH}\left(\mathrm{CH}_{3}\right)_{2}\right), 1.25(\mathrm{~m}, 4 \mathrm{H}, \mathrm{THF}), 1.21\left(\mathrm{~d},{ }^{3} J_{\mathrm{HH}}=6.8 \mathrm{~Hz}, 6 \mathrm{H}, \mathrm{CH}\left(\mathrm{CH}_{3}\right)_{2}\right), 1.17\left(\mathrm{~d},{ }^{3} J_{\mathrm{HH}}=6.8 \mathrm{~Hz}, 6 \mathrm{H}, \mathrm{CH}\left(\mathrm{CH}_{3}\right)_{2}\right)$, $0.02\left(\mathrm{~s}, 18 \mathrm{H}, \mathrm{Si}\left(\mathrm{CH}_{3}\right)_{3}\right) \mathrm{ppm} .{ }^{13} \mathrm{C}\left\{{ }^{1} \mathrm{H}\right\} \mathrm{NMR}\left(\mathrm{C}_{6} \mathrm{D}_{6}, 298 \mathrm{~K}, 125.76 \mathrm{MHz}\right): \delta 172.4(\mathrm{CH}=\mathrm{N}), 160.5\left(i-\mathrm{N}=\mathrm{CH} C_{6} \mathrm{H}_{4}\right)$, $150.0\left(i-\mathrm{NC}_{6} \mathrm{H}_{3}\right), 148.1\left(i-\mathrm{NC}_{6} \mathrm{H}_{3}\right), 144.2\left(o-\mathrm{NC}_{6} \mathrm{H}_{3}\right), 141.2\left(o-\mathrm{NC}_{6} \mathrm{H}_{3}\right), 139.4\left(C_{6} \mathrm{H}_{4}\right), 133.9\left(C_{6} \mathrm{H}_{4}\right), 126.8(p-$ $\left.\mathrm{NC}_{6} \mathrm{H}_{3}\right), 125.5\left(m-\mathrm{NC}_{6} \mathrm{H}_{3}\right), 125.1\left(p-\mathrm{NC}_{6} \mathrm{H}_{3}\right), 124.8\left(m-\mathrm{NC}_{6} \mathrm{H}_{3}\right), 120.0\left(C_{6} \mathrm{H}_{4}\right), 117.3\left(i-\mathrm{NC}_{6} \mathrm{H}_{4}\right), 112.3\left(C_{6} \mathrm{H}_{4}\right), 70.0$ (THF), $29.6\left(\mathrm{CH}\left(\mathrm{CH}_{3}\right)_{2}\right), 29.1\left(\mathrm{CH}\left(\mathrm{CH}_{3}\right)_{2}\right), 26.5\left(\mathrm{CH}\left(\mathrm{CH}_{3}\right)_{2}\right), 26.2\left(\mathrm{CH}\left(\mathrm{CH}_{3}\right)_{2}\right), 25.6(\mathrm{THF}), 25.3\left(\mathrm{CH}\left(\mathrm{CH}_{3}\right)_{2}\right), 23.7$ $\left(\mathrm{CH}\left(\mathrm{CH}_{3}\right)_{2}\right), 6.2\left(\mathrm{Si}\left(\mathrm{CH}_{3}\right)_{3}\right)$ ppm. Anal. Calc for $\mathrm{C}_{41} \mathrm{H}_{65} \mathrm{~N}_{3} \mathrm{OSi}_{2} \mathrm{Ca}\left(712.22 \mathrm{~g} \cdot \mathrm{mol}^{-1}\right): \mathrm{C} 69.14, \mathrm{H} 9.20, \mathrm{~N}$ 5.90. Found C 68.91, H 8.87, N 5.91. 


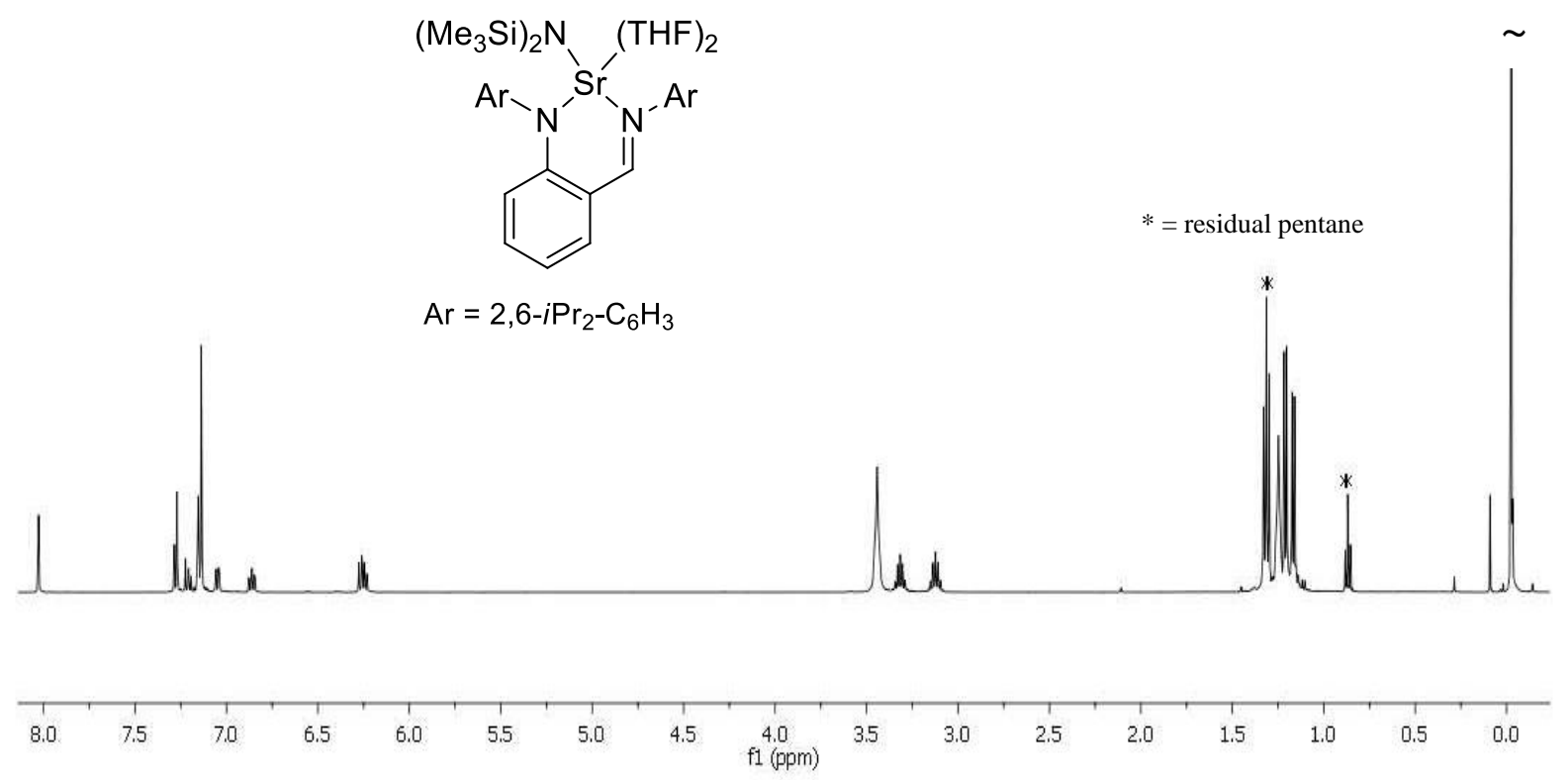

${ }^{1} \mathrm{H}$ NMR spectrum $\left(\mathrm{C}_{6} \mathrm{D}_{6}, 298 \mathrm{~K}, 500.13 \mathrm{MHz}\right)$ of 2.

$\left\{\mathrm{L}^{1}\right\} \mathrm{SrN}\left(\mathrm{SiMe}_{3}\right)_{2}(\mathrm{THF})_{2}(\mathbf{2})(0.57 \mathrm{~g}, 70 \%)$ was isolated as a yellow solid following the procedure described for $\mathbf{1}$, using $\left\{\mathrm{L}^{1}\right\} \mathrm{H}(0.43 \mathrm{~g}, 0.98 \mathrm{mmol}), \mathrm{KN}\left(\mathrm{SiMe}_{3}\right)_{2}(0.39 \mathrm{~g}, 1.96 \mathrm{mmol})$ and $\mathrm{SrI}_{2}(0.35 \mathrm{~g}, 1.03 \mathrm{mmol})$.

${ }^{1} \mathrm{H}$ NMR $\left(\mathrm{C}_{6} \mathrm{D}_{6}, 298 \mathrm{~K}, 500.13 \mathrm{MHz}\right): \delta=8.03(\mathrm{~s}, 1 \mathrm{H}, \mathrm{CH}=\mathrm{N}), 7.28\left(\mathrm{~d},{ }^{3} J_{\mathrm{HH}}=7.6 \mathrm{~Hz}, 2 \mathrm{H}\right.$, arom- $\left.H\right), 7.21\left(\mathrm{t},{ }^{3} J_{\mathrm{HH}}=\right.$ $7.6 \mathrm{~Hz}, 1 \mathrm{H}$, arom- $H), 7.14(\mathrm{~m}, 3 \mathrm{H}, \operatorname{arom}-H), 7.06\left(\mathrm{dd},{ }^{3} J_{\mathrm{HH}}=7.9 \mathrm{~Hz},{ }^{4} J_{\mathrm{HH}}=1.8 \mathrm{~Hz}, 1 \mathrm{H}\right.$, arom- $\left.H\right), 6.87\left(\mathrm{td},{ }^{3} J_{\mathrm{HH}}=\right.$ $8.0 \mathrm{~Hz},{ }^{4} J_{\mathrm{HH}}=1.8 \mathrm{~Hz}, 1 \mathrm{H}$, arom- $\left.H\right), 6.26(\mathrm{~m}, 2 \mathrm{H}$, arom- $H), 3.45(\mathrm{~m}, 8 \mathrm{H}, \mathrm{THF}), 3.32\left(\mathrm{~m}, 2 \mathrm{H}, \mathrm{CH}\left(\mathrm{CH}_{3}\right)_{2}\right), 3.13(\mathrm{~m}$, $\left.2 \mathrm{H}, \mathrm{CH}\left(\mathrm{CH}_{3}\right)_{2}\right), 1.32\left(\mathrm{~d},{ }^{3} J_{\mathrm{HH}}=7.9 \mathrm{~Hz}, 6 \mathrm{H}, \mathrm{CH}\left(\mathrm{CH}_{3}\right)_{2}\right), 1.31\left(\mathrm{~d},{ }^{3} J_{\mathrm{HH}}=7.20 \mathrm{~Hz}, 6 \mathrm{H}, \mathrm{CH}\left(\mathrm{CH}_{3}\right)_{2}\right), 1.25(\mathrm{~m}, 8 \mathrm{H}$, THF), $1.21\left(\mathrm{~d},{ }^{3} J_{\mathrm{HH}}=6.8 \mathrm{~Hz}, 6 \mathrm{H}, \mathrm{CH}\left(\mathrm{CH}_{3}\right)_{2}\right), 1.17\left(\mathrm{~d},{ }^{3} J_{\mathrm{HH}}=6.7 \mathrm{~Hz}, 6 \mathrm{H}, \mathrm{CH}\left(\mathrm{CH}_{3}\right)_{2}\right), 0.02\left(\mathrm{~s}, 18 \mathrm{H}, \mathrm{Si}\left(\mathrm{CH}_{3}\right)_{3}\right) \mathrm{ppm}$. ${ }^{13} \mathrm{C}\left\{{ }^{1} \mathrm{H}\right\}$ NMR $\left(\mathrm{C}_{6} \mathrm{D}_{6}, 298 \mathrm{~K}, 125.76 \mathrm{MHz}\right): \delta 171.2(\mathrm{CH}=\mathrm{N}), 159.5\left(i-\mathrm{N}=\mathrm{CHC} \mathrm{H}_{4}\right), 150.0\left(i-\mathrm{NC}_{6} \mathrm{H}_{3}\right), 147.2(i-$ $\left.\mathrm{NC}_{6} \mathrm{H}_{3}\right), 144.1\left(o-\mathrm{NC}_{6} \mathrm{H}_{3}\right), 141.0\left(o-\mathrm{NC}_{6} \mathrm{H}_{3}\right), 139.7\left(C_{6} \mathrm{H}_{4}\right), 133.9\left(C_{6} \mathrm{H}_{4}\right), 126.5\left(p-\mathrm{NC}_{6} \mathrm{H}_{3}\right), 125.8\left(m-\mathrm{NC}_{6} \mathrm{H}_{3}\right)$, $125.0\left(p-\mathrm{NC}_{6} \mathrm{H}_{3}\right), 124.9\left(m-\mathrm{NC}_{6} \mathrm{H}_{3}\right), 119.1\left(C_{6} \mathrm{H}_{4}\right), 117.6\left(i-\mathrm{NC}_{6} \mathrm{H}_{4}\right), 111.6\left(C_{6} \mathrm{H}_{4}\right), 69.0(\mathrm{THF}), 29.5\left(C \mathrm{H}\left(\mathrm{CH}_{3}\right)_{2}\right)$, $28.8\left(\mathrm{CH}\left(\mathrm{CH}_{3}\right)_{2}\right), 26.3\left(\mathrm{CH}\left(\mathrm{CH}_{3}\right)_{2}\right), 26.2\left(\mathrm{CH}\left(\mathrm{CH}_{3}\right)_{2}\right), 25.8(\mathrm{THF}), 25.7\left(\mathrm{CH}\left(\mathrm{CH}_{3}\right)_{2}\right), 23.9\left(\mathrm{CH}\left(\mathrm{CH}_{3}\right)_{2}\right), 6.0$ $\left(\mathrm{Si}\left(\mathrm{CH}_{3}\right)_{3}\right)$ ppm. Anal. Calc for $\mathrm{C}_{45} \mathrm{H}_{73} \mathrm{~N}_{3} \mathrm{O}_{2} \mathrm{Si}_{2} \mathrm{Sr}\left(831.87 \mathrm{~g} \cdot \mathrm{mol}^{-1}\right)$ : C 64.97, H 8.85, N 5.05. Found C 64.79, H 8.79, N 4.99. 


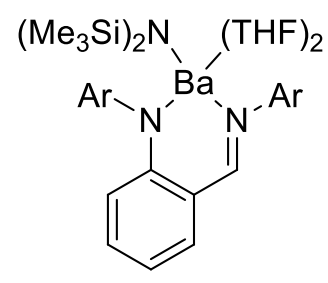

$\mathrm{Ar}=2,6-i \mathrm{Pr}_{2}-\mathrm{C}_{6} \mathrm{H}_{3}$

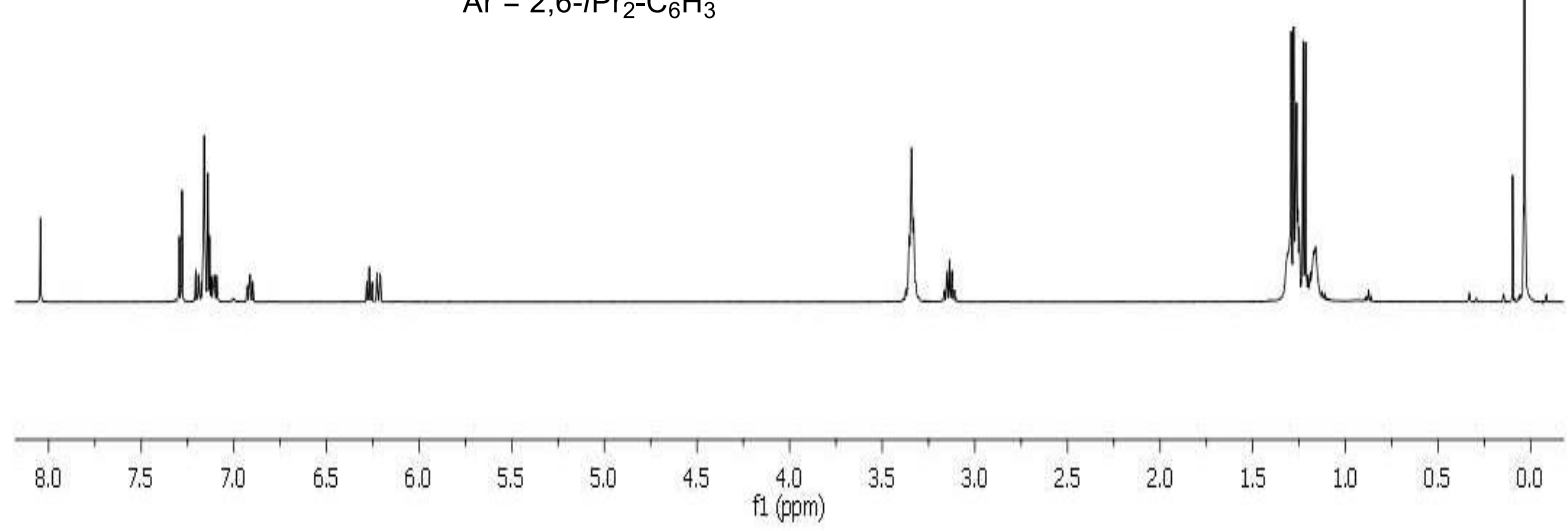

${ }^{1} \mathrm{H}$ NMR spectrum $\left(\mathrm{C}_{6} \mathrm{D}_{6}, 298 \mathrm{~K}, 500.13 \mathrm{MHz}\right)$ of $\mathbf{3}$.

$\left\{\mathrm{L}^{1}\right\} \mathrm{BaN}\left(\mathrm{SiMe}_{3}\right)_{2}(\mathrm{THF})_{2}(\mathbf{3})(0.29 \mathrm{~g}, 45 \%)$ was isolated as a yellow solid following the procedure described for $\mathbf{1}$ using $\left\{\mathrm{L}^{1}\right\} \mathrm{H}(0.32 \mathrm{~g}, 0.73 \mathrm{mmol}), \mathrm{KN}\left(\mathrm{SiMe}_{3}\right)_{2}(0.29 \mathrm{~g}, 1.46 \mathrm{mmol})$ and $\mathrm{BaI}_{2}(0.30 \mathrm{~g}, 0.77 \mathrm{mmol})$.

${ }^{1} \mathrm{H}$ NMR $\left(\mathrm{C}_{6} \mathrm{D}_{6}, 298 \mathrm{~K}, 500.13 \mathrm{MHz}\right): \delta=8.04(\mathrm{~s}, 1 \mathrm{H}, \mathrm{CH}=\mathrm{N}), 7.29\left(\mathrm{~d},{ }^{3} J_{\mathrm{HH}}=7.5 \mathrm{~Hz}, 2 \mathrm{H}\right.$, arom- $\left.H\right), 7.19\left(\mathrm{t},{ }^{3} J_{\mathrm{HH}}=\right.$ $7.2 \mathrm{~Hz}, 1 \mathrm{H}$, arom- $H), 7.14(\mathrm{~m}, 3 \mathrm{H}$, arom- $H), 7.10\left(\mathrm{dd},{ }^{3} J_{\mathrm{HH}}=7.9 \mathrm{~Hz},{ }^{4} J_{\mathrm{HH}}=1.9 \mathrm{~Hz}, 1 \mathrm{H}, \operatorname{arom}-H\right), 6.91\left(\mathrm{td},{ }^{3} J_{\mathrm{HH}}=\right.$ $8.2 \mathrm{~Hz},{ }^{4} J_{\mathrm{HH}}=1.9 \mathrm{~Hz}, 1 \mathrm{H}$, arom- $\left.H\right), 6.27\left(\mathrm{td},{ }^{3} J_{\mathrm{HH}}=8.3 \mathrm{~Hz},{ }^{4} J_{\mathrm{HH}}=1.2 \mathrm{~Hz}, 1 \mathrm{H}\right.$, arom- $\left.H\right), 6.22\left(\mathrm{~d},{ }^{3} J_{\mathrm{HH}}=8.3 \mathrm{~Hz}, 1 \mathrm{H}\right.$, arom- $H), 3.34\left(\mathrm{~m}, 8 \mathrm{H}, \mathrm{THF}+2 \mathrm{H}, \mathrm{CH}\left(\mathrm{CH}_{3}\right)_{2}\right), 3.14\left(\mathrm{~m}, 2 \mathrm{H}, \mathrm{CH}\left(\mathrm{CH}_{3}\right)_{2}\right), 1.31\left(\mathrm{br}, 6 \mathrm{H}, \mathrm{CH}\left(\mathrm{CH}_{3}\right)_{2}\right), 1.29\left(\mathrm{~d},{ }^{3} \mathrm{~J}_{\mathrm{HH}}=\right.$ $\left.7.10 \mathrm{~Hz}, 6 \mathrm{H}, \mathrm{CH}\left(\mathrm{CH}_{3}\right)_{2}\right), 1.27$ (multi, 8H, THF), 1.22 (d, $\left.{ }^{3} \mathrm{~J}_{\mathrm{HH}}=6.7 \mathrm{~Hz}, 6 \mathrm{H}, \mathrm{CH}\left(\mathrm{CH}_{3}\right)_{2}\right), 1.16$ (br, 6H, $\left.\mathrm{CH}\left(\mathrm{CH}_{3}\right)_{2}\right)$, $0.03\left(\mathrm{~s}, 18 \mathrm{H}, \mathrm{Si}\left(\mathrm{CH}_{3}\right)_{3}\right) \mathrm{ppm} .{ }^{13} \mathrm{C}\left\{{ }^{1} \mathrm{H}\right\} \mathrm{NMR}\left(\mathrm{C}_{6} \mathrm{D}_{6}, 298 \mathrm{~K}, 125.76 \mathrm{MHz}\right): \delta 169.3(\mathrm{CH}=\mathrm{N}), 158.0\left(i-\mathrm{N}=\mathrm{CH} C_{6} \mathrm{H}_{4}\right)$, $149.8\left(i-\mathrm{NC}_{6} \mathrm{H}_{3}\right), 146.5\left(i-\mathrm{NC}_{6} \mathrm{H}_{3}\right), 144.6\left(o-\mathrm{NC}_{6} \mathrm{H}_{3}\right), 141.0\left(o-\mathrm{NC}_{6} \mathrm{H}_{3}\right), 139.6\left(C_{6} \mathrm{H}_{4}\right), 133.9\left(C_{6} \mathrm{H}_{4}\right), 126.1(p-$ $\left.\mathrm{NC}_{6} \mathrm{H}_{3}\right), 126.0\left(m-\mathrm{NC}_{6} \mathrm{H}_{3}\right), 124.9\left(p-\mathrm{NC}_{6} \mathrm{H}_{3}, m-\mathrm{NC}_{6} \mathrm{H}_{3}\right), 118.6\left(C_{6} \mathrm{H}_{4}\right), 118.5\left(i-\mathrm{NC}_{6} \mathrm{H}_{4}\right), 111.1\left(C_{6} \mathrm{H}_{4}\right), 68.6(\mathrm{THF})$, $29.4\left(\mathrm{CH}\left(\mathrm{CH}_{3}\right)_{2}\right), 28.8\left(\mathrm{CH}\left(\mathrm{CH}_{3}\right)_{2}\right), 26.4\left(\mathrm{CH}\left(\mathrm{CH}_{3}\right)_{2}\right), 26.2\left(\mathrm{CH}\left(\mathrm{CH}_{3}\right)_{2}\right), 25.9$ (THF), $25.7\left(\mathrm{CH}\left(\mathrm{CH}_{3}\right)_{2}\right), 24.0$ $\left(\mathrm{CH}\left(\mathrm{CH}_{3}\right)_{2}\right), 5.8\left(\mathrm{Si}\left(\mathrm{CH}_{3}\right)_{3}\right)$ ppm. Anal. Calc for $\mathrm{C}_{45} \mathrm{H}_{73} \mathrm{~N}_{3} \mathrm{O}_{2} \mathrm{Si}_{2} \mathrm{Ba}\left(881.58 \mathrm{~g} \cdot \mathrm{mol}^{-1}\right): \mathrm{C}$ 61.31, H 8.35, N 4.77. Found C 61.20, H 8.28, N 4.72. 


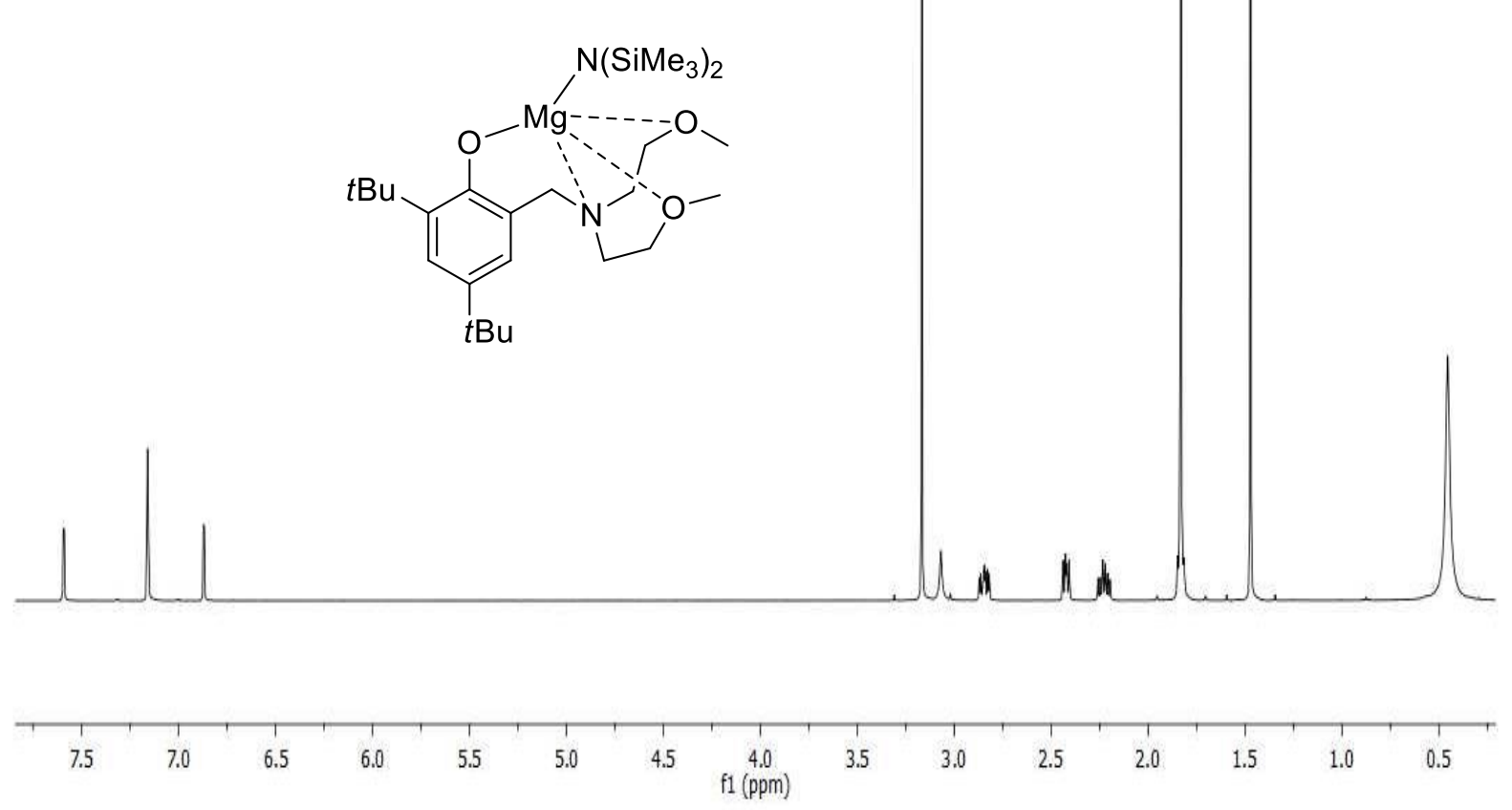

${ }^{1} \mathrm{H}$ NMR spectrum $\left(\mathrm{C}_{6} \mathrm{D}_{6}, 298 \mathrm{~K}, 500.13 \mathrm{MHz}\right)$ of 4.

At room temperature, a solution of $\left\{\mathrm{L}^{2}\right\} \mathrm{H}(0.22 \mathrm{~g}, 0.63 \mathrm{mmol})$ in pentane $(5 \mathrm{~mL})$ was added slowly to a pentane $(10 \mathrm{~mL})$ solution of $\mathrm{Mg}\left[\mathrm{N}\left(\mathrm{SiMe}_{3}\right)_{2}\right]_{2}(0.23 \mathrm{~g}, 0.66 \mathrm{mmol})$. The solution was then stirred at room temperature. After $6 \mathrm{~h}$, the reaction solution was concentrated gradually in vacuo and $\mathbf{4}$ precipitated as a white solid which was isolated by filtration $(0.25 \mathrm{~g}, 75 \%)$. Crystals suitable for single-crystal X-ray diffraction crystallography were obtained by storing a concentrated pentane solution at $-26{ }^{\circ} \mathrm{C}$ several days. ${ }^{1} \mathrm{H}$ NMR $\left(\mathrm{C}_{6} \mathrm{D}_{6}, 298 \mathrm{~K}, 500.13 \mathrm{MHz}\right): \delta$ $7.59\left(\mathrm{~d},{ }^{4} J_{\mathrm{HH}}=2.5 \mathrm{~Hz}, 1 \mathrm{H}\right.$, arom- $\left.H\right), 6.87\left(\mathrm{~d},{ }^{4} J_{\mathrm{HH}}=2.5 \mathrm{~Hz}, 1 \mathrm{H}\right.$, arom- $\left.H\right), 3.17\left(\mathrm{~s}, 6 \mathrm{H}, \mathrm{O}-\mathrm{CH}_{3}\right), 3.07(\mathrm{~s}, 2 \mathrm{H}, \mathrm{Ar}-$ $\left.\mathrm{CH}_{2}-\mathrm{N}\right), 2.84\left(\mathrm{~m}, 2 \mathrm{H}, \mathrm{CH}_{2}-\mathrm{C}(H) \mathrm{H}-\mathrm{O}\right), 2.42\left(\mathrm{~m}, 2 \mathrm{H}, \mathrm{CH}_{2}-\mathrm{C}(H) \mathrm{H}-\mathrm{O}\right), 2.23\left(\mathrm{~m}, 2 \mathrm{H}, \mathrm{N}-\mathrm{CH}_{2}-\mathrm{CH}_{2}\right), 1.83(\mathrm{br}, 2 \mathrm{H}, \mathrm{N}-$ $\left.\mathrm{CH}_{2}-\mathrm{CH}_{2}+9 \mathrm{H}, o-\mathrm{C}\left(\mathrm{CH}_{3}\right)_{3}\right), 1.47\left(\mathrm{~s}, 9 \mathrm{H}, p-\mathrm{C}\left(\mathrm{CH}_{3}\right)_{3}\right), 0.46\left(\mathrm{~s}, 18 \mathrm{H}, \mathrm{Si}\left(\mathrm{CH}_{3}\right)_{2}\right) \mathrm{ppm} .{ }^{13} \mathrm{C}\left\{{ }^{1} \mathrm{H}\right\} \mathrm{NMR}\left(\mathrm{C}_{6} \mathrm{D}_{6}, 298 \mathrm{~K}\right.$, 125.76 MHz): $\delta 164.9$ (i-C), $138.3(o-\mathrm{C}), 134.7$ (p-C), $124.92(o-\mathrm{C}), 124.8(m-\mathrm{C}), 122.9(m-\mathrm{C}), 69.1\left(\mathrm{CH}_{2}-\mathrm{CH}_{2}-\mathrm{O}\right)$, $60.2\left(\mathrm{Ar}-\mathrm{CH}_{2}-\mathrm{N}\right), 60.0\left(\mathrm{O}-\mathrm{CH}_{3}\right), 56.6\left(\mathrm{~N}-\mathrm{CH}_{2}-\mathrm{CH}_{2}\right), 36.3\left(o-C\left(\mathrm{CH}_{3}\right)_{3}\right), 34.6\left(p-C\left(\mathrm{CH}_{3}\right)_{3}\right), 32.9\left(p-\mathrm{C}\left(C \mathrm{H}_{3}\right)_{3}\right), 31.1$ $\left(o-\mathrm{C}\left(\mathrm{CH}_{3}\right)_{3}\right), 7.0\left(\mathrm{Si}\left(\mathrm{CH}_{3}\right)_{3}\right)$ ppm. Anal. Calc for $\mathrm{C}_{27} \mathrm{H}_{54} \mathrm{~N}_{2} \mathrm{O}_{3} \mathrm{Si}_{2} \mathrm{Mg}\left(535.21 \mathrm{~g} \cdot \mathrm{mol}^{-1}\right): \mathrm{C} 60.59, \mathrm{H} \mathrm{10.17}$, N 5.23. Found C 60.69, H 9.99, N 5.36. 


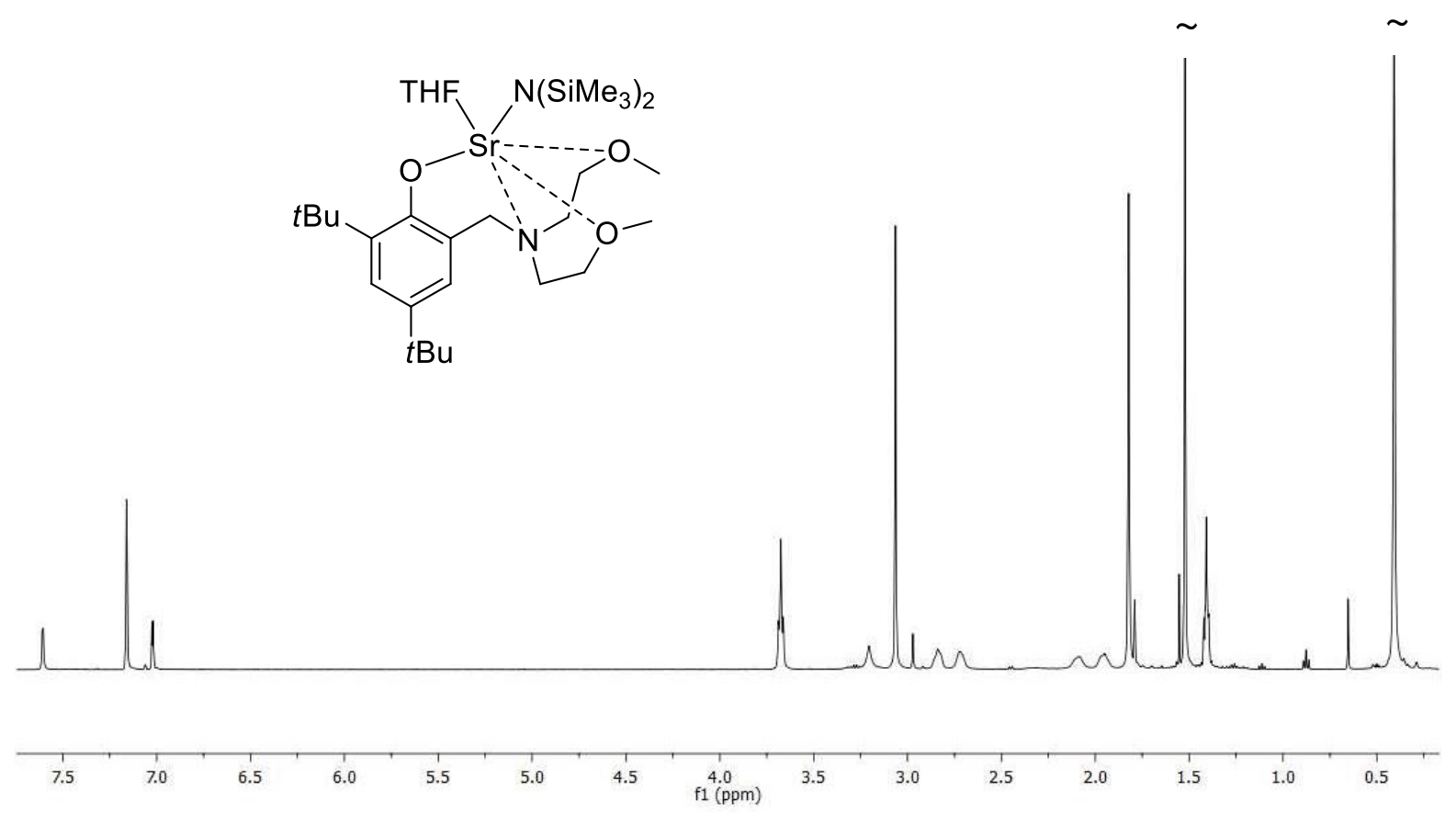

${ }^{1} \mathrm{H}$ NMR spectrum $\left(\mathrm{C}_{6} \mathrm{D}_{6}, 298 \mathrm{~K}, 500.13 \mathrm{MHz}\right)$ of 6.

$\left\{\mathrm{L}^{2}\right\} \mathrm{SrN}\left(\mathrm{SiMe}_{3}\right)_{2}(\mathrm{THF})(\mathbf{6})(0.45 \mathrm{~g}, 70 \%)$ was isolated as a colorless solid following the procedure described for 4 using $\left\{\mathrm{L}^{2}\right\} \mathrm{H}(0.34 \mathrm{~g}, 0.97 \mathrm{mmol})$ and $\mathrm{Sr}\left[\mathrm{N}\left(\mathrm{SiMe}_{3}\right)_{2}\right]_{2}(\mathrm{THF})_{2}(0.56 \mathrm{~g}, 1.02 \mathrm{mmol})$.

${ }^{1} \mathrm{H}$ NMR $\left(\mathrm{C}_{6} \mathrm{D}_{6}, 298 \mathrm{~K}, 500.13 \mathrm{MHz}\right): \delta 7.61\left(\mathrm{~d},{ }^{4} J_{\mathrm{HH}}=2.6 \mathrm{~Hz}, 1 \mathrm{H}\right.$, arom- $\left.H\right), 7.02\left(\mathrm{~d},{ }^{4} J_{\mathrm{HH}}=2.6 \mathrm{~Hz}, 1 \mathrm{H}\right.$, arom- $\left.H\right)$, 3.67 (m, 4H, THF), 3.21 (s, 2H, Ar- $\mathrm{CH}_{2}-\mathrm{N}$ ), 3.06 (s, 6H, O-CH3), 2.84 (br, 2H, $\left.\mathrm{CH}_{2}-\mathrm{C}(H) \mathrm{H}-\mathrm{O}\right), 2.72$ (br, 2H, $\mathrm{CH}_{2-}$ $\mathrm{C}(H) \mathrm{H}-\mathrm{O}), 2.08$ (br, 2H, N-CH$\left.-\mathrm{CH}_{2}\right), 1.95\left(\mathrm{br}, 2 \mathrm{H}, \mathrm{N}-\mathrm{CH}_{2}-\mathrm{CH}_{2}\right), 1.82\left(\mathrm{~s}, 9 \mathrm{H}, o-\mathrm{C}\left(\mathrm{CH}_{3}\right)_{3}\right), 1.52\left(\mathrm{~s}, 9 \mathrm{H}, p-\mathrm{C}\left(\mathrm{CH}_{3}\right)_{3}\right)$, 1.42 (m, 4H, THF), 0.41(s, 18H, Si $\left.\left(\mathrm{CH}_{3}\right)_{2}\right)$ ppm. ${ }^{13} \mathrm{C}\left\{{ }^{1} \mathrm{H}\right\}$ NMR $\left(\mathrm{C}_{6} \mathrm{D}_{6}, 298 \mathrm{~K}, 125.76 \mathrm{MHz}\right): \delta 166.2(i-\mathrm{C}), 137.0$ (o-C), 132.3 (p-C), $126.5(o-C), 124.5(m-\mathrm{C}), 122.4(m-\mathrm{C}), 69.9\left(\mathrm{CH}_{2}-\mathrm{CH}_{2}-\mathrm{O}\right), 69.3(\mathrm{THF}), 60.6\left(\mathrm{Ar}-\mathrm{CH}_{2}-\mathrm{N}\right), 60.3$ $\left(\mathrm{O}-\mathrm{CH}_{3}\right), 53.4\left(\mathrm{~N}-\mathrm{CH}_{2}-\mathrm{CH}_{2}\right), 36.2\left(o-C\left(\mathrm{CH}_{3}\right)_{3}\right), 34.6\left(p-C\left(\mathrm{CH}_{3}\right)_{3}\right), 33.0\left(p-\mathrm{C}\left(\mathrm{CH}_{3}\right)_{3}\right), 30.8\left(o-\mathrm{C}\left(\mathrm{CH}_{3}\right)_{3}\right), 25.8(\mathrm{THF})$, $6.7\left(\mathrm{Si}\left(\mathrm{CH}_{3}\right)_{2} \mathrm{H}\right)$ ppm. Anal. Calc for $\mathrm{C}_{31} \mathrm{H}_{62} \mathrm{~N}_{2} \mathrm{O}_{4} \mathrm{Si}_{2} \mathrm{Sr}\left(670.33 \mathrm{~g} \cdot \mathrm{mol}^{-1}\right)$ : C 55.52, H 9.32, N 4.18. Found C 55.42, H 9.07, N 4.23. 


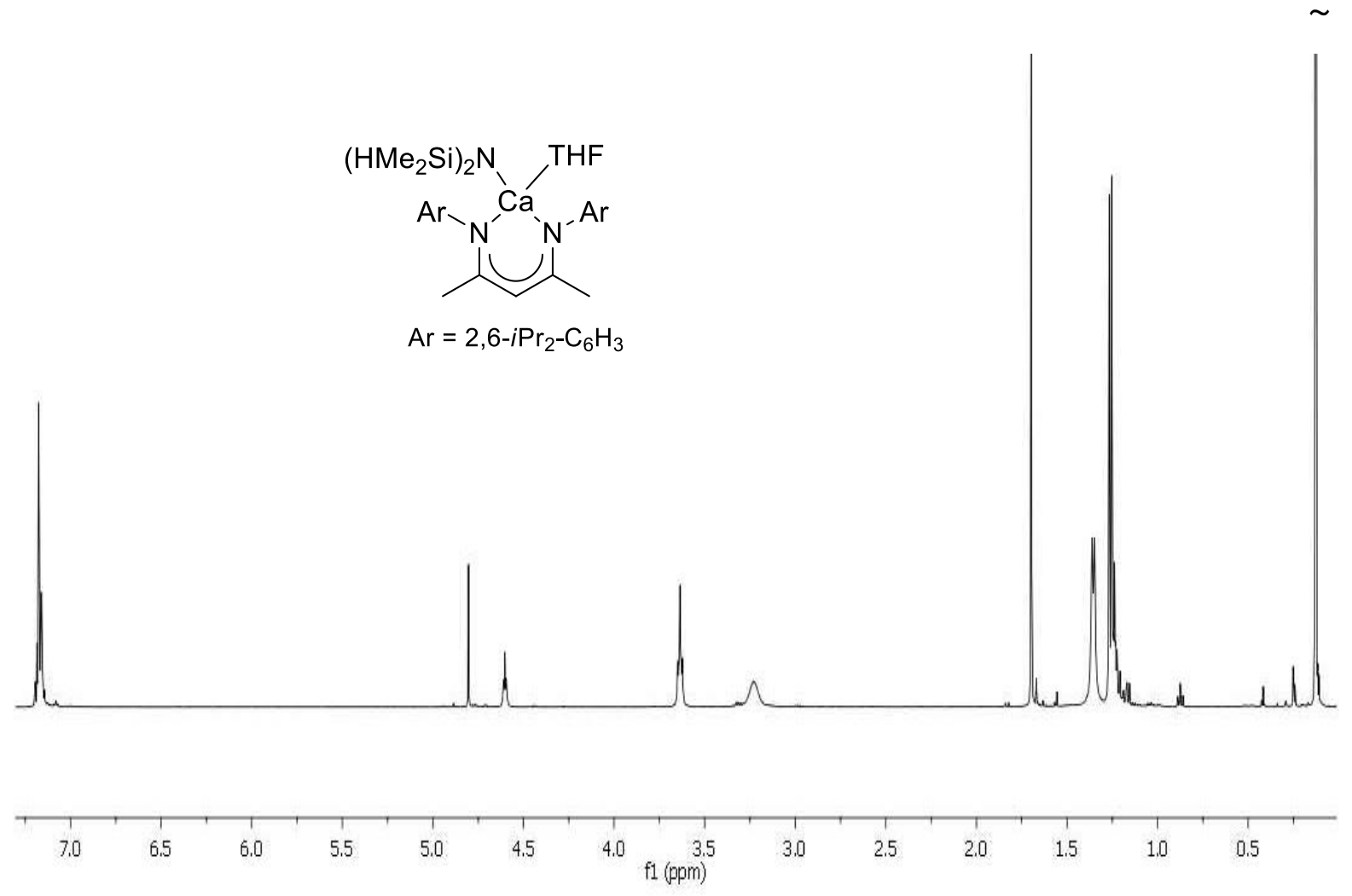

${ }^{1} \mathrm{H}$ NMR spectrum $\left(\mathrm{C}_{6} \mathrm{D}_{6}, 298 \mathrm{~K}, 500.13 \mathrm{MHz}\right)$ of $\mathbf{8}$.

The $\beta$-diimine pro-ligand $\left\{\mathrm{L}^{3}\right\} \mathrm{H}(0.55 \mathrm{~g}, 1.31 \mathrm{mmol})$ and $\mathrm{KN}\left(\mathrm{SiMe}_{2} \mathrm{H}\right)_{2}(0.45 \mathrm{~g}, 2.63 \mathrm{mmol})$ were dissolved in THF $(20 \mathrm{~mL})$. After the mixture was stirred for $1 \mathrm{~h}$, it was added to a suspension of $\mathrm{CaI}_{2}(0.40 \mathrm{~g}, 1.36 \mathrm{mmol})$ in 10 $\mathrm{mL}$ of THF and the resulting mixture was stirred for $2.5 \mathrm{~h}$. Removal of the solvent under vacuum, extraction of the residue by filtration with $50 \mathrm{~mL}$ pentane and evaporation of the volatile fraction after filtration yielded $\mathbf{8}(0.66 \mathrm{~g}$, $76 \%)$ as a colorless solid. Crystals suitable for single-crystal X-ray crystallography were obtained by storage of a concentrated pentane solution at $-26{ }^{\circ} \mathrm{C}$ overnight. ${ }^{1} \mathrm{H}$ NMR $\left(\mathrm{C}_{6} \mathrm{D}_{6}, 298 \mathrm{~K}, 500.13 \mathrm{MHz}\right): \delta 7.14(\mathrm{~m}, 6 \mathrm{H}$, arom- $H)$, $4.79(\mathrm{~s}, 1 \mathrm{H}, \mathrm{MeCCHCMe}), 4.59\left(\mathrm{~m},{ }^{1} J_{\mathrm{SiH}}=163 \mathrm{~Hz}, 2 \mathrm{H}, \mathrm{SiMe}_{2} H\right), 3.62(\mathrm{~m}, 4 \mathrm{H}, \mathrm{THF}), 3.25\left(\mathrm{~m}, 4 \mathrm{H}, \mathrm{CH}\left(\mathrm{CH}_{3}\right)_{2}\right)$, $1.68\left(\mathrm{~s}, 6 \mathrm{H}, \mathrm{CH}_{3} \mathrm{CCHCCH}_{3}\right), 1.33\left(\mathrm{~d},{ }^{3} \mathrm{~J}_{\mathrm{HH}}=7.0 \mathrm{~Hz}, 12 \mathrm{H}, \mathrm{CH}\left(\mathrm{CH}_{3}\right)_{2}\right), 1.24\left(\mathrm{~d},{ }^{3} J_{\mathrm{HH}}=7.0 \mathrm{~Hz}, 12 \mathrm{H}, \mathrm{CH}\left(\mathrm{CH}_{3}\right)_{2}\right)$, $1.17(\mathrm{~m}, 4 \mathrm{H}, \mathrm{THF}), 0.11\left(\mathrm{~d},{ }^{3} \mathrm{~J}_{\mathrm{HH}}=3 \mathrm{~Hz}, 12 \mathrm{H}, \mathrm{Si}\left(\mathrm{CH}_{3}\right)_{2} \mathrm{H}\right) \mathrm{ppm} .{ }^{13} \mathrm{C}\left\{{ }^{1} \mathrm{H}\right\} \mathrm{NMR}\left(\mathrm{C}_{6} \mathrm{D}_{6}, 298 \mathrm{~K}, 125.76 \mathrm{MHz}\right): \delta$ $166.6(C(\mathrm{Me})=\mathrm{N}), 147.0(i-C), 142.0(o-C), 125.2(p-C), 124.5(m-C), 93.9$ (MeCCHCMe), 69.8 (THF), 28.9 $\left(\mathrm{CH}\left(\mathrm{CH}_{3}\right)_{2}\right), 25.8\left(\mathrm{CH}\left(\mathrm{CH}_{3}\right)_{2}\right), 25.7$ (THF), $25.0\left(\mathrm{CH}_{3} \mathrm{CCHCCH}_{3}\right), 4.9\left(\mathrm{Si}\left(\mathrm{CH}_{3}\right)_{2} \mathrm{H}\right) \mathrm{ppm} .{ }^{29} \mathrm{Si}\left\{{ }^{1} \mathrm{H}\right\} \mathrm{NMR}\left(\mathrm{C}_{6} \mathrm{D}_{6}\right.$, $298 \mathrm{~K}, 79.49 \mathrm{MHz}$ ): $\delta-24.0$ ppm. IR (Nujol in KBr plates): $v 2029$ (m), 1959 (w), 1928 (sh) $\mathrm{cm}^{-1}$. Anal. Calc. for $\mathrm{C}_{37} \mathrm{H}_{63} \mathrm{CaN}_{3} \mathrm{OSi}_{2}\left(662.16 \mathrm{~g} \cdot \mathrm{mol}^{-1}\right)$ : C, 67.11; H, 9.59; N, 6.35. Found: C, 67.10; H, 9.48; N, 6.31. 


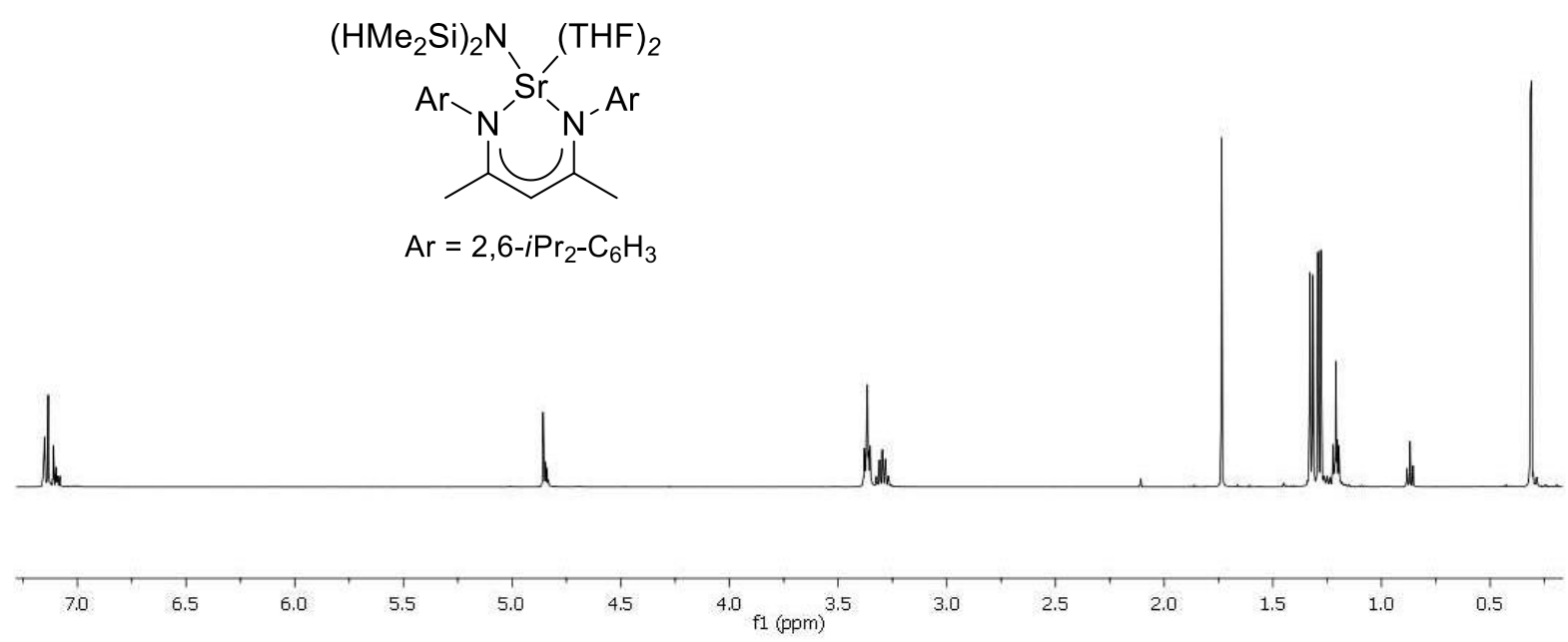

${ }^{1} \mathrm{H}$ NMR spectrum $\left(\mathrm{C}_{6} \mathrm{D}_{6}, 298 \mathrm{~K}, 500.13 \mathrm{MHz}\right)$ of 9.

$\left\{\mathrm{L}^{3}\right\} \mathrm{SrN}\left(\mathrm{SiMe}_{3}\right)_{2}(\mathrm{THF})_{2}(\mathbf{9})(0.69 \mathrm{~g}, 78 \%)$ was isolated as a colorless solid following the procedure described for 8 using $\left\{\mathrm{L}^{3}\right\} \mathrm{H}(0.48 \mathrm{~g}, 1.14 \mathrm{mmol}), \mathrm{KN}\left(\mathrm{SiMe}_{2} \mathrm{H}\right)_{2}(0.40 \mathrm{~g}, 2.28 \mathrm{mmol})$ and $\operatorname{SrI}_{2}(0.41 \mathrm{~g}, 1.20 \mathrm{mmol})$.

${ }^{1} \mathrm{H}$ NMR $\left(\mathrm{C}_{6} \mathrm{D}_{6}, 298 \mathrm{~K}, 500.13 \mathrm{MHz}\right): \delta 7.16-7.11(\mathrm{~m}, 6 \mathrm{H}$, arom- $H), 4.87(\mathrm{~s}, 1 \mathrm{H}, \mathrm{MeCC} H \mathrm{CMe}), 4.86\left(\mathrm{~m},{ }^{1} J_{\mathrm{SiH}}=\right.$ $\left.160 \mathrm{~Hz}, 2 \mathrm{H}, \mathrm{SiMe}_{2} H\right), 3.37$ (m, 8H, THF), $3.32\left(\mathrm{~m}, 4 \mathrm{H}, \mathrm{CH}\left(\mathrm{CH}_{3}\right)_{2}\right), 1.74\left(\mathrm{~s}, 6 \mathrm{H}, \mathrm{CH}_{3} \mathrm{CCHCCH}_{3}\right), 1.33\left(\mathrm{~d},{ }^{3} J_{\mathrm{HH}}=\right.$ $\left.7.0 \mathrm{~Hz}, 12 \mathrm{H}, \mathrm{CH}\left(\mathrm{CH}_{3}\right)_{2}\right), 1.29\left(\mathrm{~d},{ }^{3} J_{\mathrm{HH}}=7.0 \mathrm{~Hz}, 12 \mathrm{H}, \mathrm{CH}\left(\mathrm{CH}_{3}\right)_{2}\right), 1.22(\mathrm{~m}, 8 \mathrm{H}, \mathrm{THF}), 0.33\left(\mathrm{~d},{ }^{3} J_{\mathrm{HH}}=3.0 \mathrm{~Hz}, 12 \mathrm{H}\right.$, $\left.\mathrm{Si}\left(\mathrm{CH}_{3}\right)_{2} \mathrm{H}\right)$ ppm. ${ }^{13} \mathrm{C}\left\{{ }^{1} \mathrm{H}\right\}$ NMR $\left(\mathrm{C}_{6} \mathrm{D}_{6}, 298 \mathrm{~K}, 125.76 \mathrm{MHz}\right): \delta 165.3(C(\mathrm{Me})=\mathrm{N}), 148.3(i-C), 141.9(o-C), 124.5$ (p-C), 124.3 (m-C), 93.4 (MeCCHCMe), 69.9 (THF), $28.8\left(\mathrm{CH}\left(\mathrm{CH}_{3}\right)_{2}\right), 25.9\left(\mathrm{CH}\left(\mathrm{CH}_{3}\right)_{2}\right), 25.7$ (THF), 25.0 $\left(\mathrm{CH}_{3} \mathrm{CCHCCH}_{3}\right), 5.4\left(\mathrm{Si}\left(\mathrm{CH}_{3}\right)_{2} \mathrm{H}\right) \mathrm{ppm} .{ }^{29} \mathrm{Si}\left\{{ }^{1} \mathrm{H}\right\}$ NMR $\left(\mathrm{C}_{6} \mathrm{D}_{6}, 298 \mathrm{~K}, 79.49 \mathrm{MHz}\right): \delta-26.3 \mathrm{ppm}$. IR (Nujol in $\mathrm{KBr}$ plates): v 2025 (s), 1959 (sh), 1909 (w) cm $\mathrm{cm}^{-1}$. Anal. Calc. for $\mathrm{C}_{41} \mathrm{H}_{71} \mathrm{SrN}_{3} \mathrm{O}_{2} \mathrm{Si}_{2}\left(781.81 \mathrm{~g} \cdot \mathrm{mol}^{-1}\right): \mathrm{C}, 62.99 ; \mathrm{H}$, 9.15; N, 5.37. Found: C, 62.81; H, 9.08; N, 5.28. 


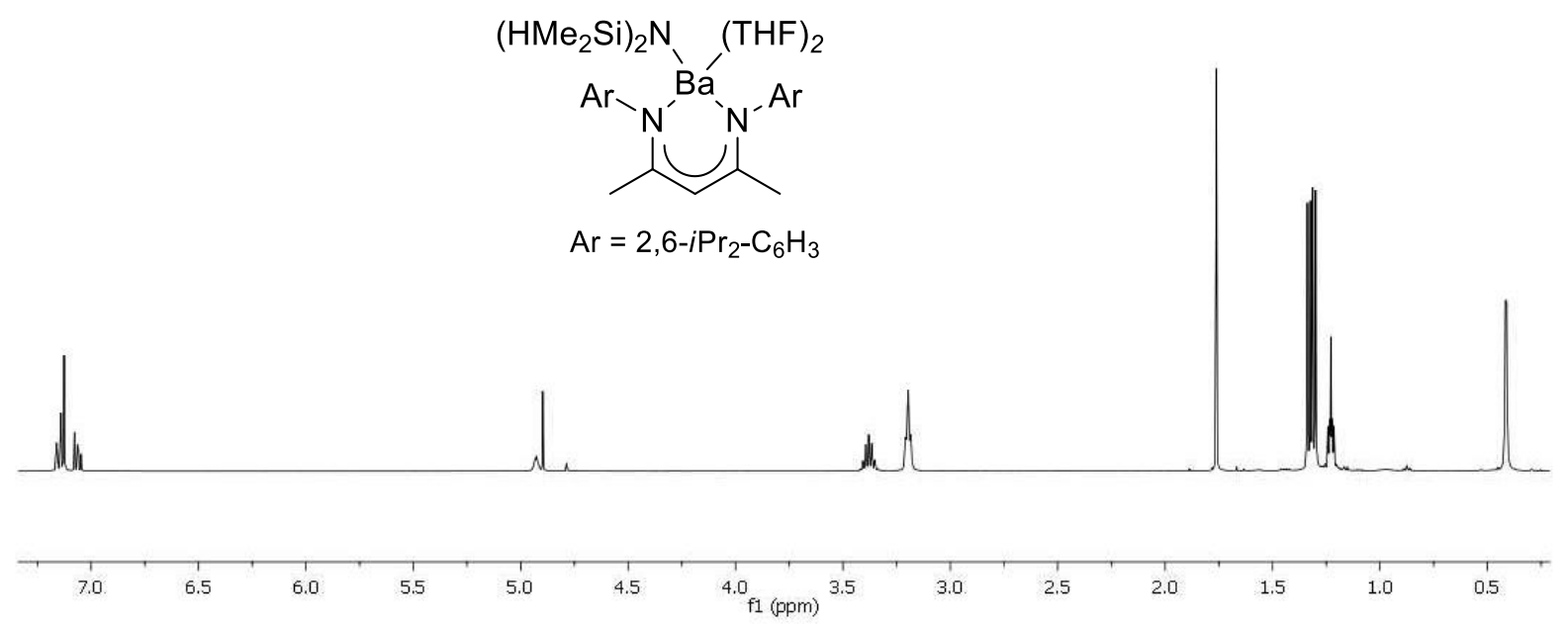

${ }^{1} \mathrm{H}$ NMR spectrum $\left(\mathrm{C}_{6} \mathrm{D}_{6}, 298 \mathrm{~K}, 500.13 \mathrm{MHz}\right)$ of $\mathbf{1 0}$.

$\left\{\mathrm{L}^{3}\right\} \mathrm{BaN}\left(\mathrm{SiMe}_{3}\right)_{2}(\mathrm{THF})_{2}(\mathbf{1 0})(0.43 \mathrm{~g}, 74 \%)$ was isolated as a colorless solid following the procedure described for 8 using $\left\{\mathrm{L}^{3}\right\} \mathrm{H}(0.29 \mathrm{~g}, 0.70 \mathrm{mmol}), \mathrm{KN}\left(\mathrm{SiMe}_{2} \mathrm{H}\right)_{2}(0.24 \mathrm{~g}, 1.40 \mathrm{mmol})$ and $\mathrm{BaI}_{2}(0.29 \mathrm{~g}, 0.74 \mathrm{mmol})$.

${ }^{1} \mathrm{H}$ NMR $\left(\mathrm{C}_{6} \mathrm{D}_{6}, 298 \mathrm{~K}, 500.13 \mathrm{MHz}\right): \delta 7.13\left(\mathrm{~d},{ }^{3} J_{\mathrm{HH}}=7.2 \mathrm{~Hz}, 4 \mathrm{H}\right.$, arom- $\left.H\right), 7.06\left(\mathrm{t},{ }^{3} J_{\mathrm{HH}}=7.2 \mathrm{~Hz}, 2 \mathrm{H}\right.$, arom- $\left.H\right)$, $4.93\left(\mathrm{~m},{ }^{1} J_{\mathrm{SiH}}=159 \mathrm{~Hz}, 2 \mathrm{H}, \mathrm{SiMe}_{2} H\right), 4.90(\mathrm{~s}, 1 \mathrm{H}, \mathrm{MeCCHCMe}), 3.38$ (m, 4H, CH(CH$\left.)_{2}\right), 3.20(\mathrm{~m}, 8 \mathrm{H}, \mathrm{THF})$, $1.76\left(\mathrm{~s}, 6 \mathrm{H}, \mathrm{CH}_{3} \mathrm{CCHC} \mathrm{CH}_{3}\right), 1.33\left(\mathrm{~d},{ }^{3} J_{\mathrm{HH}}=7.0 \mathrm{~Hz}, 12 \mathrm{H}, \mathrm{CH}\left(\mathrm{CH}_{3}\right)_{2}\right), 1.30\left(\mathrm{~d},{ }^{3} J_{\mathrm{HH}}=7.0 \mathrm{~Hz}, 12 \mathrm{H}, \mathrm{CH}\left(\mathrm{CH}_{3}\right)_{2}\right)$, $1.23(\mathrm{~m}, 8 \mathrm{H}, \mathrm{THF}), 0.41\left(\mathrm{~d},{ }^{3} J_{\mathrm{HH}}=3.0 \mathrm{~Hz}, 12 \mathrm{H}, \mathrm{Si}\left(\mathrm{CH}_{3}\right)_{2} \mathrm{H}\right) \mathrm{ppm} .{ }^{13} \mathrm{C}\left\{{ }^{1} \mathrm{H}\right\} \mathrm{NMR}\left(\mathrm{C}_{6} \mathrm{D}_{6}, 298 \mathrm{~K}, 125.76 \mathrm{MHz}\right): \delta$ $163.3(C(\mathrm{Me})=\mathrm{N}), 148.4\left(i-\mathrm{NC}_{6} \mathrm{H}_{3}\right), 142.2\left(o-\mathrm{NC}_{6} \mathrm{H}_{3}\right), 124.3\left(p-\mathrm{NC}_{6} \mathrm{H}_{3}\right), 124.0\left(m-\mathrm{NC}_{6} \mathrm{H}_{3}\right), 94.0(\mathrm{MeCCHCMe})$, 68.4 (THF), $28.6\left(\mathrm{CH}\left(\mathrm{CH}_{3}\right)_{2}\right), 26.2\left(\mathrm{CH}\left(\mathrm{CH}_{3}\right)_{2}\right), 25.8$ (THF), $25.1\left(\mathrm{CH}_{3} \mathrm{CCHCCH}_{3}\right), 5.1\left(\mathrm{Si}\left(\mathrm{CH}_{3}\right)_{2} \mathrm{H}\right) \mathrm{ppm} .{ }^{29} \mathrm{Si}\left\{{ }^{1} \mathrm{H}\right\}$ NMR (C $\mathrm{C}_{6} \mathrm{D}_{6}, 298 \mathrm{~K}, 79.49 \mathrm{MHz}$ ): $\delta$-28.9 ppm. IR (Nujol in KBr plates): $v 2009$ (s), $1913(\mathrm{~m}), 1859$ (w) cm $\mathrm{cm}^{-1}$. Anal. Calc. for $\mathrm{C}_{41} \mathrm{H}_{71} \mathrm{BaN}_{3} \mathrm{O}_{2} \mathrm{Si}_{2}\left(831.52 \mathrm{~g} \cdot \mathrm{mol}^{-1}\right)$ : C, 59.22; H, 8.61; N, 5.05. Found: C, 58.84; H, 8.51; N, 4.91 . 


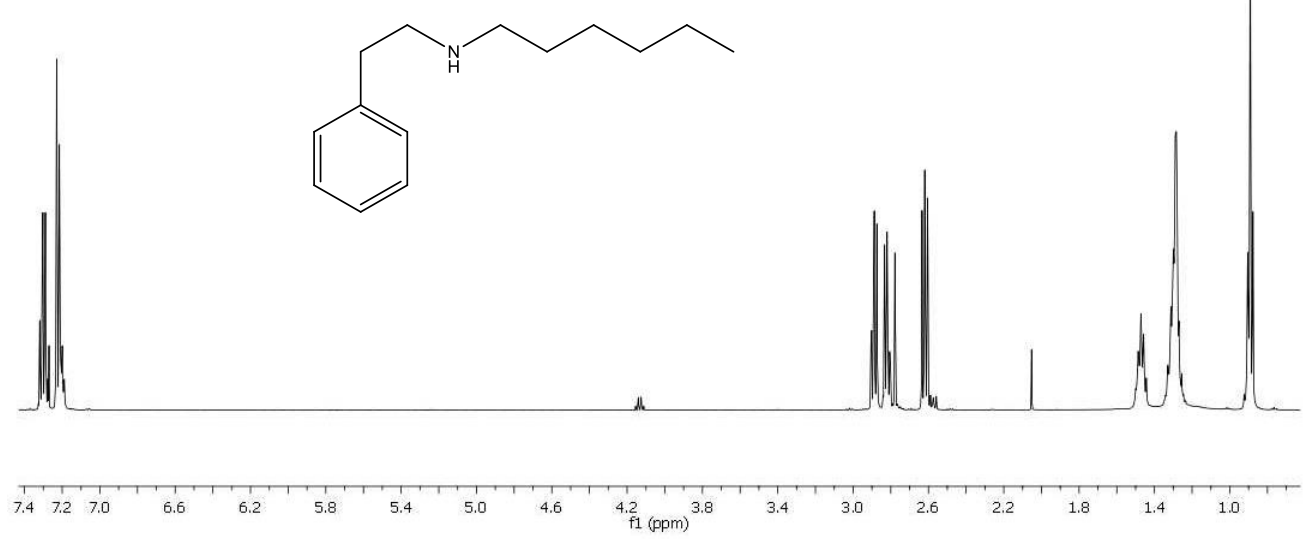

${ }^{1} \mathrm{H}$ NMR $\left(\mathrm{CDCl}_{3}, 298 \mathrm{~K}, 500.13 \mathrm{MHz}\right): \delta=7.15(\mathrm{~m}, 2 \mathrm{H}$, arom- $H), 7.08(\mathrm{~m}, 3 \mathrm{H}$, arom- $H), 2.73(\mathrm{~m}, 2 \mathrm{H}$, $\mathrm{NCH}(H) \mathrm{CH}(H) \mathrm{Ph}), 2.67(\mathrm{~m}, 2 \mathrm{H}, \mathrm{NCH}(H) \mathrm{CH}(H) \mathrm{Ph}), 2.47\left(\mathrm{t},{ }^{3} J_{\mathrm{HH}}=7.3 \mathrm{~Hz}, 2 \mathrm{H}, \mathrm{NCH}_{2} \mathrm{CH}_{2}\left(\mathrm{CH}_{2}\right)_{3} \mathrm{CH}_{3}\right), 1.32(\mathrm{~m}$, $\left.2 \mathrm{H}, \mathrm{NCH}_{2} \mathrm{CH}_{2}\left(\mathrm{CH}_{2}\right)_{3} \mathrm{CH}_{3}\right), 1.13\left(\mathrm{~m}, 6 \mathrm{H}, \mathrm{NCH}_{2} \mathrm{CH}_{2}\left(\mathrm{CH}_{2}\right)_{3} \mathrm{CH}_{3}, 1 \mathrm{H}, \mathrm{NH}\right), 0.72$ (t, ${ }^{3} J_{\mathrm{HH}}=6.9 \mathrm{~Hz}, 3 \mathrm{H}$, $\left.\mathrm{NCH}_{2} \mathrm{CH}_{2}\left(\mathrm{CH}_{2}\right)_{3} \mathrm{CH}_{3}\right)$ ppm. ${ }^{13} \mathrm{C}\left\{{ }^{1} \mathrm{H}\right\} \mathrm{NMR}\left(\mathrm{CDCl}_{3}, 298 \mathrm{~K}, 125.76 \mathrm{MHz}\right): \delta=140.6(i-\mathrm{C}), 129.2(m-\mathrm{C}), 128.9(o-$

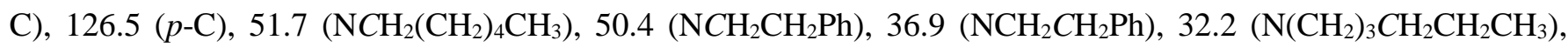

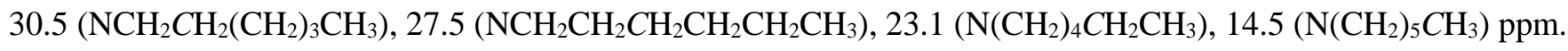

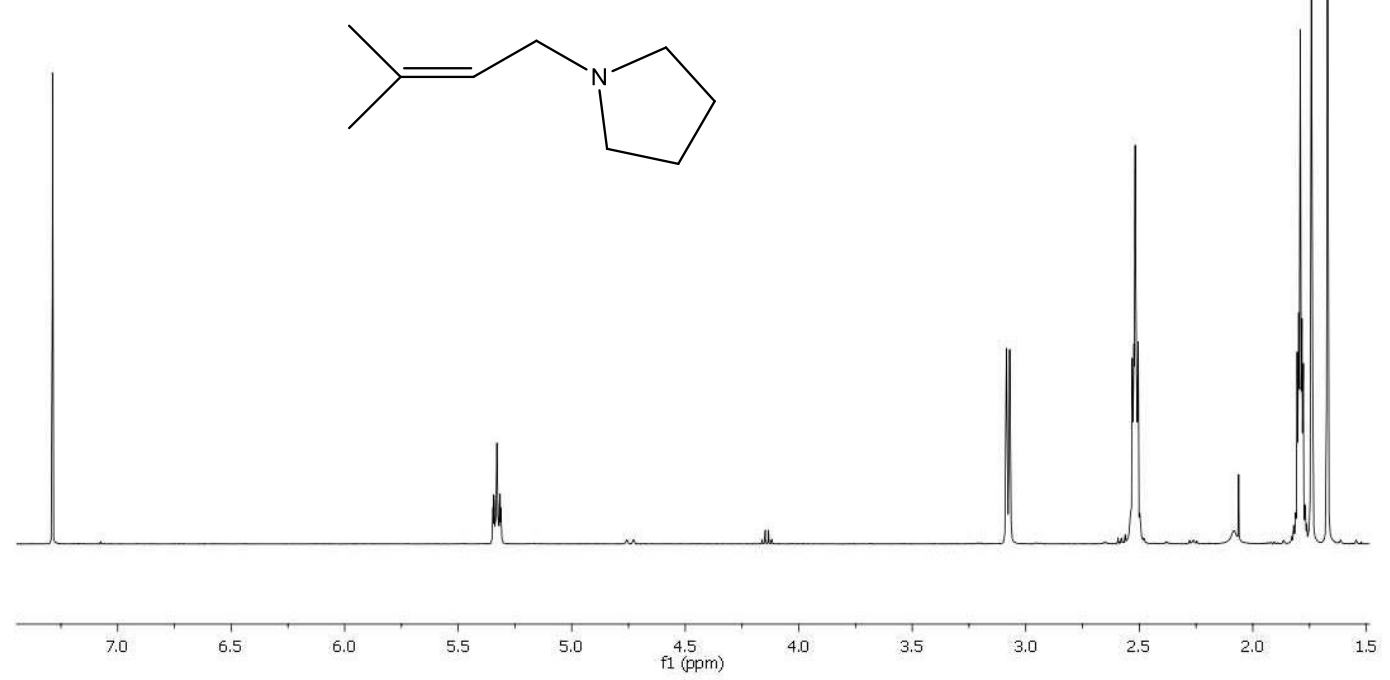

${ }^{1} \mathrm{H} \mathrm{NMR}\left(\mathrm{CDCl}_{3}, 298 \mathrm{~K}, 500.13 \mathrm{MHz}\right): \delta=5.31\left(\mathrm{~m}, 1 \mathrm{H}, \mathrm{C}=\mathrm{CHCH}_{2}\right), 3.06\left(\mathrm{~d},{ }^{3} J_{\mathrm{HH}}=7.0 \mathrm{~Hz}, 2 \mathrm{H}, \mathrm{C}=\mathrm{CHCH}_{2}\right), 2.50$ (m, 4H, $\left.\mathrm{NCH}_{2} \mathrm{CH}_{2}\right), 1.77$ (m, 4H, $\left.\mathrm{NCH}_{2} \mathrm{CH}_{2}\right), 1.72$ (s, 3H, $\left.\mathrm{CH}_{3} \mathrm{C}=\mathrm{CH}\right), 1.65$ (s, 3H, CH $\left.\mathrm{C}_{3} \mathrm{C}=\mathrm{CH}\right) \mathrm{ppm} ;{ }^{13} \mathrm{C}\left\{{ }^{1} \mathrm{H}\right\}$ $\mathrm{NMR}\left(\mathrm{CDCl}_{3}, 298 \mathrm{~K}, 125.76 \mathrm{MHz}\right): \delta=134.3\left(\mathrm{C}=\mathrm{CHCH}_{2}\right), 122.6\left(\mathrm{C}=\mathrm{CHCH}_{2}\right), 54.5\left(\mathrm{NCH}_{2} \mathrm{CH}_{2}\right), 54.3$ $\left(\mathrm{C}=\mathrm{CHCH}_{2}\right), 26.3\left(\mathrm{CH}_{3} \mathrm{C}=\mathrm{CH}\right), 23.9\left(\mathrm{NCH}_{2} \mathrm{CH}_{2}\right), 18.4\left(\mathrm{CH}_{3} \mathrm{C}=\mathrm{CH}\right) \mathrm{ppm}$. 


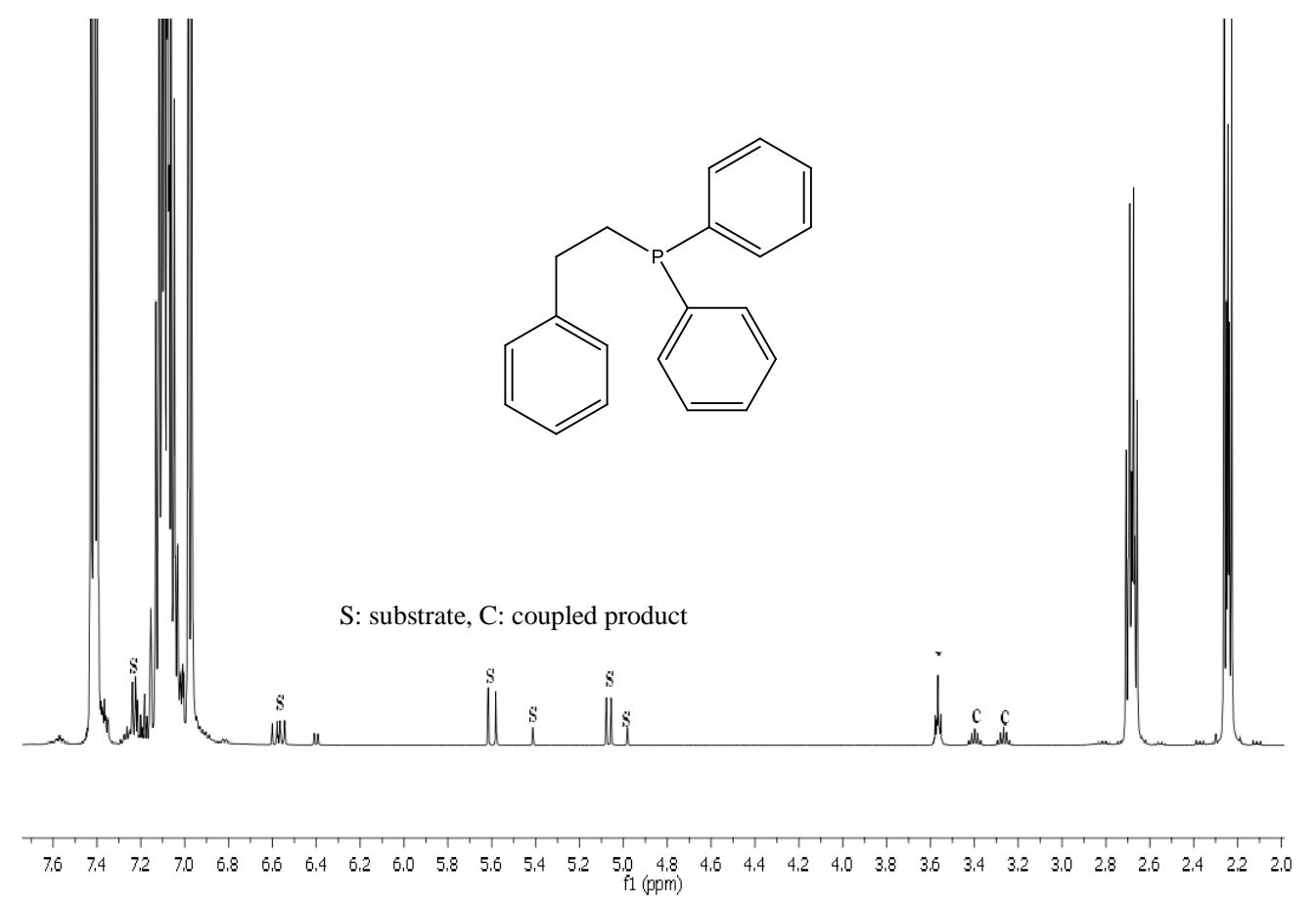

${ }^{1} \mathrm{H}$ NMR $\left(\mathrm{C}_{6} \mathrm{D}_{6}, 298 \mathrm{~K}, 500.13 \mathrm{MHz}\right): \delta=7.42(\mathrm{~m}, 4 \mathrm{H}$, arom- $H), 7.14-7.04$ (m, 9H, arom- $\left.H\right), 6.99$ (m, 2H, aromH) $2.72\left(\mathrm{~m}, 2 \mathrm{H}, \mathrm{PCH}_{2} \mathrm{CH}_{2}\right), 2.25\left(\mathrm{~m}, 2 \mathrm{H}, \mathrm{PCH}_{2} \mathrm{CH}_{2}\right) \mathrm{ppm} .{ }^{13} \mathrm{C}\left\{{ }^{1} \mathrm{H}\right\} \mathrm{NMR}\left(\mathrm{C}_{6} \mathrm{D}_{6}, 298 \mathrm{~K}, 125.76 \mathrm{MHz}\right): \delta=143.4$, 139.9, 133.6, 129.3, 129.2, 129.1, 129.0, $33.2\left(\mathrm{PCH}_{2} \mathrm{CH}_{2}\right), 31.2\left(\mathrm{PCH}_{2} \mathrm{CH}_{2}\right)$ ppm. ${ }^{31} \mathrm{P}\left\{{ }^{1} \mathrm{H}\right\} \mathrm{NMR}\left(\mathrm{C}_{6} \mathrm{D}_{6}, 298 \mathrm{~K}\right.$, $161.96 \mathrm{MHz}): \delta=-2.7 \mathrm{ppm}$.

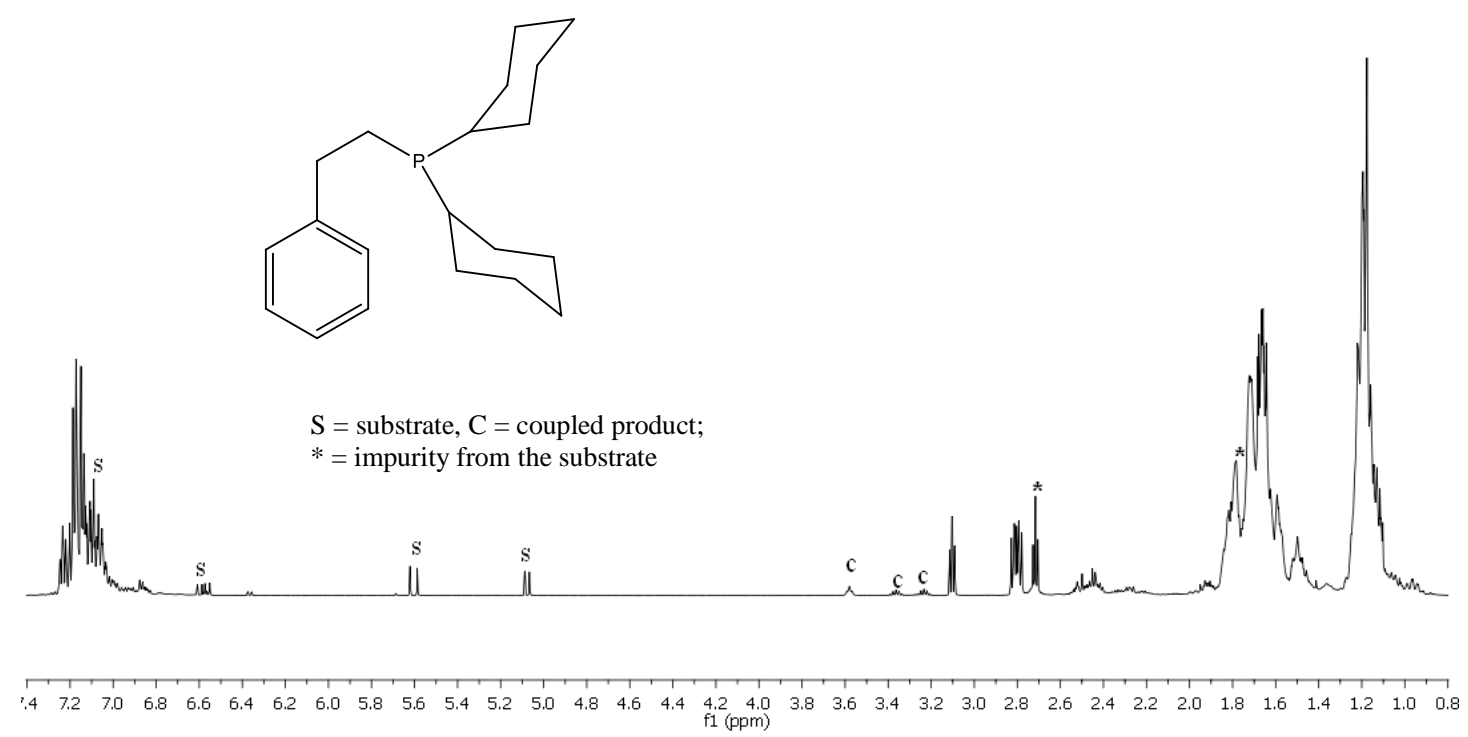

${ }^{1} \mathrm{H}$ NMR $\left(\mathrm{C}_{6} \mathrm{D}_{6}, 298 \mathrm{~K}, 500.13 \mathrm{MHz}\right): \delta=7.22(\mathrm{~m}, 2 \mathrm{H}$, arom- $H), 7.6-7.04(\mathrm{~m}, 3 \mathrm{H}$, arom- $H), 2.79(\mathrm{~m}, 2 \mathrm{H}$, $\left.\mathrm{PCH}_{2} \mathrm{CH}_{2}\right), 2.45\left(\mathrm{~m}, 2 \mathrm{H}, \mathrm{PCH}_{2} \mathrm{CH}_{2}\right), 1.63(\mathrm{~m}, 10 \mathrm{H}, \mathrm{PCy}), 1.21(\mathrm{~m}, 12 \mathrm{H}, \mathrm{PCy}) \mathrm{ppm} .{ }^{13} \mathrm{C}\left\{{ }^{1} \mathrm{H}\right\} \mathrm{NMR}\left(\mathrm{C}_{6} \mathrm{D}_{6}, 298 \mathrm{~K}\right.$, 125.76 MHz): $\delta=147.3,144.4,143.1,127.0,36.1(C y), 34.5\left(\mathrm{PCH}_{2} \mathrm{CH}_{2}\right), 34.0(\mathrm{PCy}), 33.12(\mathrm{PCy}), 30.6$ $\left(\mathrm{PCH}_{2} \mathrm{CH}_{2}\right) 29.8$ (PCy) ppm. ${ }^{31} \mathrm{P}$ NMR $\left(\mathrm{C}_{6} \mathrm{D}_{6}, 298 \mathrm{~K}, 161.96 \mathrm{MHz}\right): \delta=-3.1 \mathrm{ppm}$. 


\section{S11 Typical protocol for intermolecular hydrofunctionalization reactions}

Catalytic intermolecular hydroamination reactions were carried out using the following standard procedure. In the glovebox, $9.3 \mathrm{mg}(10.5 \mu \mathrm{mol})$ of $\left\{\mathrm{L}^{1}\right\} \mathrm{BaN}\left(\mathrm{SiMe}_{3}\right)_{2}(\mathrm{THF})_{2}(3)$ was loaded into an NMR tube. The NMR tube was stored in an appropriate Schlenk tube, and it was removed from the glove-box. The subsequent manipulations were performed using standard Schlenk techniques. Styrene (61 $\mu \mathrm{L}, 525 \mu \mathrm{mol})$ and benzyl amine (58 $\mu \mathrm{L}, 525$ $\mu \mathrm{mol}$ ) were added to the NMR tube using microsyringes. The NMR tube was sealed and shaken vigorously, then put into an oil bath at $60{ }^{\circ} \mathrm{C}$. The reaction times were measured from this point. After the required amount of time, the reaction was quenched and $\mathrm{C}_{6} \mathrm{D}_{6}$ was added to the mixture at room temperature. The conversion was determined according to the ${ }^{1} \mathrm{H}$ NMR spectrum of the reaction mixture.

A similar protocol was followed for intermolecular hydrophosphination reactions.

S12 X-ray structure of $\left\{\mathrm{L}^{1}\right\} \mathrm{CaN}\left(\mathrm{SiMe}_{3}\right)_{2}(\mathrm{THF})(1)$

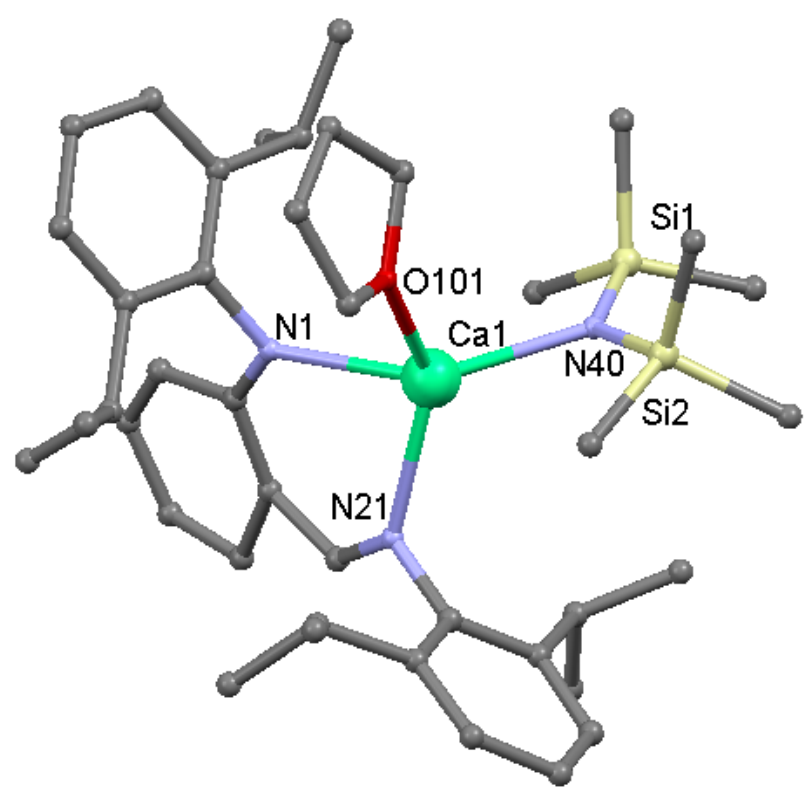

Representation of the molecular solid-state structure of $\left\{\mathrm{L}^{1}\right\} \mathrm{CaN}\left(\mathrm{SiMe}_{3}\right)_{2}(\mathrm{THF})$ (1). Only the main site for the disordered aromatic ring is depicted. Hydrogen atoms omitted for clarity. 
S13 X-ray structure of $\left\{\mathrm{L}^{2}\right\} \mathrm{MgN}\left(\mathrm{SiMe}_{3}\right)_{2}(4)$

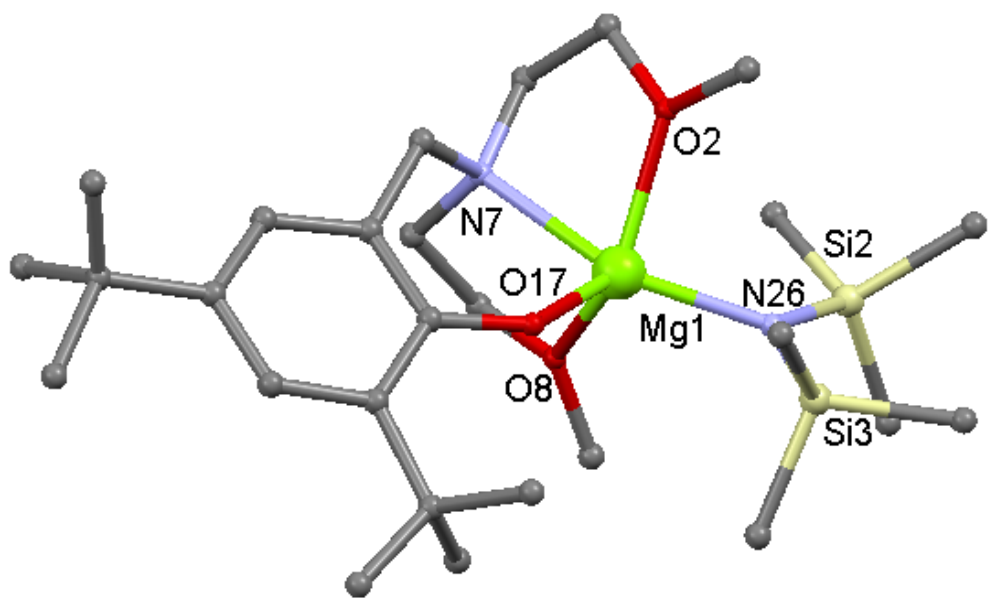

Representation of the molecular solid-state structure of $\left\{\mathrm{L}^{2}\right\} \mathrm{MgN}\left(\mathrm{SiMe}_{3}\right)_{2}$ (4). Hydrogen atoms and non-interacting solvent molecule (benzene) omitted for clarity. Only the main site of the disordered $t \mathrm{Bu}$ group is depicted.

S14 X-ray structure of $\left\{\mathrm{L}^{2}\right\} \operatorname{SrN}\left(\mathrm{SiMe}_{3}\right)_{2}(\mathrm{THF})(6)$

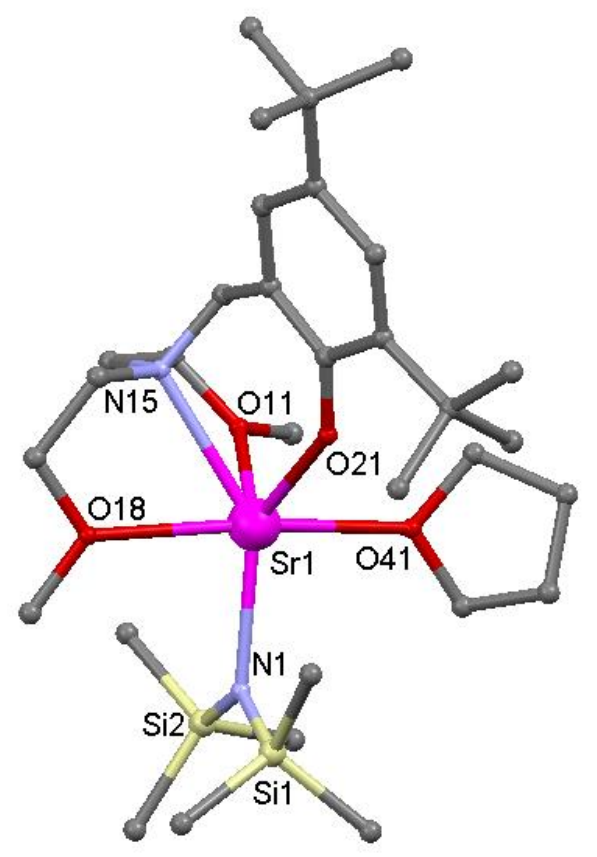

Representation of the molecular solid-state structure of $\left\{\mathrm{L}^{2}\right\} \mathrm{SrN}\left(\mathrm{SiMe}_{3}\right)_{2}(\mathrm{THF})(6)$. Hydrogen atoms omitted for clarity. 
S15 X-ray structure of $\left\{\mathrm{L}^{3}\right\} \mathrm{CaN}\left(\mathrm{SiMe}_{2} \mathrm{H}\right)_{2}(\mathrm{THF})(8)$

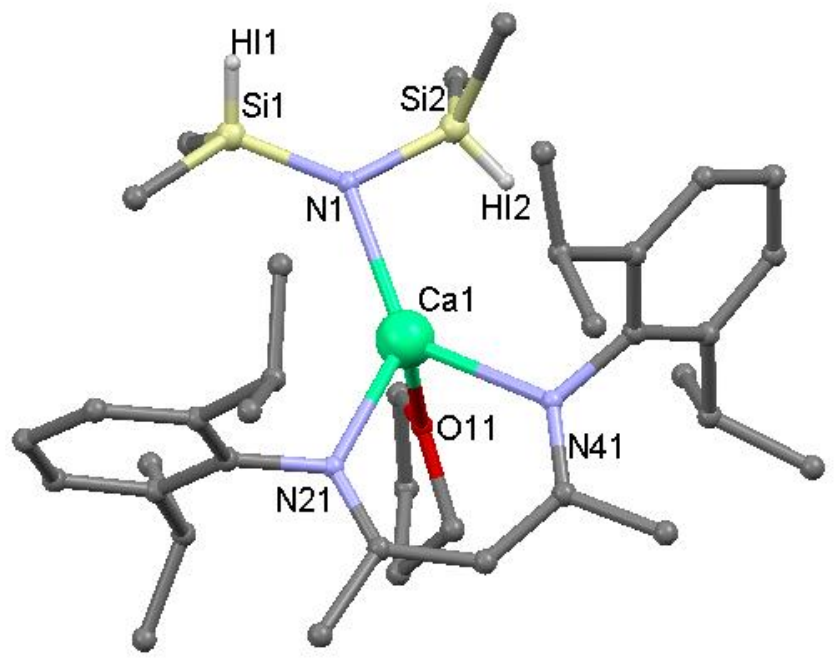

Representation of the molecular solid-state structure of $\left\{\mathrm{L}^{3}\right\} \mathrm{CaN}\left(\mathrm{SiMe}_{3}\right)_{2}(\mathrm{THF})$ (8). Only one of the two independent molecules is displayed. Hydrogen atoms omitted for clarity.

S16 X-ray structure of $\left\{\mathrm{L}^{3}\right\} \mathrm{BaN}\left(\mathrm{SiMe}_{2} \mathrm{H}\right)_{2}(\mathrm{THF})_{2}(10)$

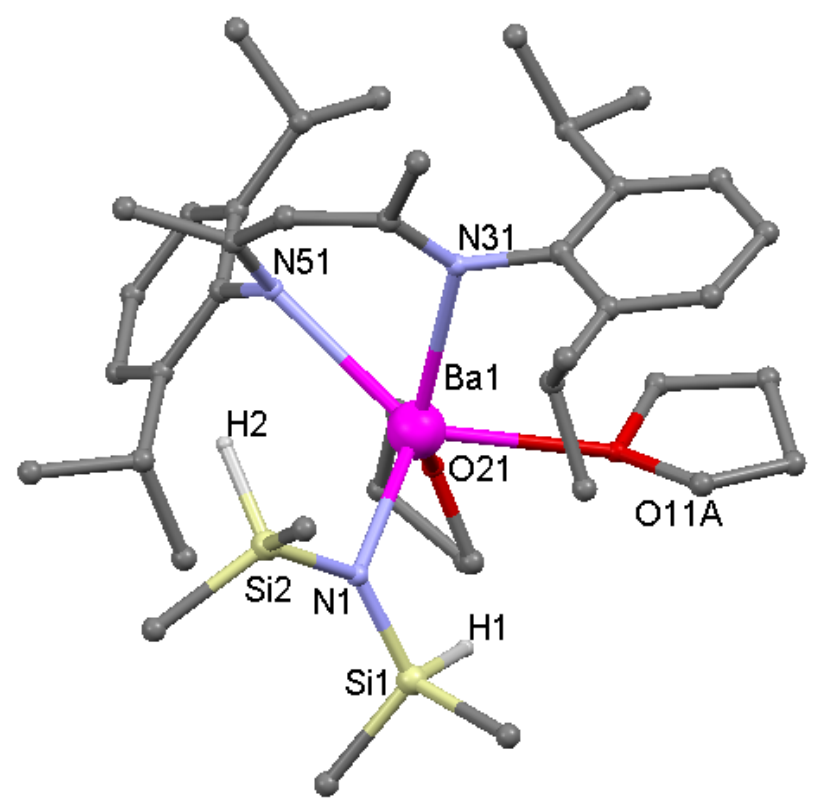

Representation of the molecular solid-state structure of $\left\{\mathrm{L}^{3}\right\} \mathrm{BaN}\left(\mathrm{SiMe}_{3}\right)_{2}(\mathrm{THF})_{2}(\mathbf{1 0})$. Hydrogen atoms (except those on silicon atoms) omitted for clarity. Only the main site of the disordered THF molecule is depicted. 


\begin{tabular}{|c|c|c|c|}
\hline & 1 & 3 & 4 \\
\hline Empirical formula & $\mathrm{C}_{41} \mathrm{H}_{65} \mathrm{CaN}_{3} \mathrm{OSi}_{2}$ & $\mathrm{C}_{45} \mathrm{H}_{73} \mathrm{BaN}_{3} \mathrm{O}_{2} \mathrm{Si}_{2}$ & $\mathrm{C}_{30} \mathrm{H}_{57} \mathrm{MgN}_{2} \mathrm{O}_{3} \mathrm{Si}_{2}$ \\
\hline CCDC number & 862063 & 862065 & 862066 \\
\hline Formula weight & 712.22 & 881.58 & 574.27 \\
\hline Crystal system & Monoclinic & Triclinic & Monoclinic \\
\hline Space group & $\mathrm{P} 22_{1} / \mathrm{n}$ & P -1 & $\mathrm{P} 2{ }_{1} / \mathrm{n}$ \\
\hline$a, \AA$ & $11.3665(4)$ & $10.1126(6)$ & $11.7436(7)$ \\
\hline$b, \AA$ & $18.7618(6)$ & $13.3068(8)$ & $10.8108(4)$ \\
\hline$c, \AA$ & $19.9250(6)$ & $19.2267(12)$ & $27.5157(14)$ \\
\hline$\alpha, \operatorname{deg}$ & 90 & $84.951(2)$ & 90 \\
\hline$\beta, \operatorname{deg}$ & $91.2970(10)$ & $89.783(2)$ & $94.384(2)$ \\
\hline$\gamma, \operatorname{deg}$ & 90 & $67.887(2)$ & 90 \\
\hline Volume, $\AA^{3}$ & $4248.0(2)$ & 2386.4(3) & $3483.1(3)$ \\
\hline$Z$ & 4 & 2 & 4 \\
\hline Density, $\mathrm{g} . \mathrm{cm}^{-3}$ & 1.112 & 1.227 & 1.095 \\
\hline Abs. coeff., mm ${ }^{-1}$ & 0.237 & 0.918 & 0.150 \\
\hline$F(000)$ & 1548 & 928 & 1260 \\
\hline Crystal size, mm & $0.37 \times 0.32 \times 0.15$ & $0.27 \times 0.17 \times 0.10$ & $0.27 \times 0.24 \times 0.20$ \\
\hline \multirow[t]{2}{*}{$\theta$ range, deg } & 2.96 to 27.48 & 2.99 to 27.60 & 2.92 to 27.49 \\
\hline & $-12<\mathrm{h}<14$ & $-12<\mathrm{h}<12$ & $-15<\mathrm{h}<15$ \\
\hline \multirow[t]{2}{*}{ Limiting indices } & $-24<\mathrm{k}<22$ & $-17<\mathrm{k}<16$ & $-14<\mathrm{k}<11$ \\
\hline & $-18<1<25$ & $-24<1<24$ & $-35<1<34$ \\
\hline Rint & 0.0523 & 0.0502 & 0.0478 \\
\hline Reflec. collected & 37979 & 37845 & 30361 \\
\hline Reflec. Unique $[I>2 \sigma(I)]$ & 9736 & 10665 & 7973 \\
\hline Data/restraints/param. & $9736 / 15 / 473$ & 10665 / 0 / 481 & 7973 / 0 / 388 \\
\hline Goodness-of-fit on $F^{2}$ & 1.030 & 1.019 & 1.049 \\
\hline$R_{1}[I>2 \sigma(I)]$ (all data) & $0.0819(0.2001)$ & $0.0436(0.0942)$ & $0.0409(0.0986)$ \\
\hline $\mathrm{w} R_{2}[I>2 \sigma(I)]$ (all data) & $0.1349(0.2282)$ & $0.0644(0.1051)$ & $0.0574(0.1071)$ \\
\hline Largest diff. e $\mathrm{A}^{-3}$ & 0.999 and -0.783 & 0.902 and -0.851 & 0.274 and -0.251 \\
\hline
\end{tabular}




\begin{tabular}{|c|c|c|c|}
\hline & 6 & 8 & 10 \\
\hline Empirical formula & $\mathrm{C}_{31} \mathrm{H}_{62} \mathrm{~N}_{2} \mathrm{O}_{4} \mathrm{Si}_{2} \mathrm{Sr}$ & $\mathrm{C}_{74} \mathrm{H}_{126} \mathrm{Ca}_{2} \mathrm{~N}_{6} \mathrm{O}_{2} \mathrm{Si}_{4}$ & $\mathrm{C}_{41} \mathrm{H}_{71} \mathrm{BaN}_{3} \mathrm{O}_{2} \mathrm{Si}_{2}$ \\
\hline CCDC number & 862067 & 862068 & 862064 \\
\hline Formula weight & 670.63 & 1324.33 & 831.53 \\
\hline Crystal system & Triclinic & Triclinic & Triclinic \\
\hline Space group & P -1 & $\mathrm{P}-1$ & $\mathrm{P}-1$ \\
\hline$a, \AA$ & $9.7687(8)$ & $12.6363(18)$ & $10.7704(4)$ \\
\hline$b, \AA$ & $13.9830(10)$ & $16.297(2)$ & $11.5967(5)$ \\
\hline$c, \AA$ & $14.8454(8)$ & 19.954(3) & $20.4220(8)$ \\
\hline$\alpha, \operatorname{deg}$ & $68.657(2)$ & $81.362(7)$ & $90.889(2)$ \\
\hline$\beta, \operatorname{deg}$ & $78.581(3)$ & $83.707(7)$ & $94.2040(10)$ \\
\hline$\gamma, \operatorname{deg}$ & $87.360(3)$ & $90.019(7)$ & $109.8940(10)$ \\
\hline Volume, $\AA^{3}$ & $1850.6(2)$ & 4037.5(10) & 2389.83(17) \\
\hline$Z$ & 2 & 2 & 2 \\
\hline Density, $\mathrm{g} . \mathrm{cm}^{-3}$ & 1.203 & 1.089 & 1.156 \\
\hline Abs. coeff., $\mathrm{mm}^{-1}$ & 1.555 & 0.244 & 0.913 \\
\hline$F(000)$ & 720 & 1448 & 876 \\
\hline Crystal size, mm & $0.20 \times 0.20 \times 0.20$ & $0.48 \times 0.29 \times 0.11$ & $0.57 \times 0.36 \times 0.28$ \\
\hline$\theta$ range, deg & 3.47 to 27.47 & 1.26 to 27.45 & 2.94 to 27.48 \\
\hline \multirow[t]{3}{*}{ Limiting indices } & $-12<\mathrm{h}<12$ & $-16<\mathrm{h}<16$ & $-13<\mathrm{h}<13$ \\
\hline & $-18<\mathrm{k}<13$ & $-20<\mathrm{k}<21$ & $-15<\mathrm{k}<15$ \\
\hline & $-19<1<19$ & $0<1<25$ & $-26<1<26$ \\
\hline Rint & 0.0452 & 0.0000 & 0.0342 \\
\hline Reflec. collected & 30098 & 14363 & 35806 \\
\hline Reflec. Unique $[I>2 \sigma(I)]$ & 8342 & 14363 & 10713 \\
\hline Data/restraints/param. & $8342 / 0 / 375$ & $14363 / 0 / 822$ & $10713 / 0$ / 444 \\
\hline Goodness-of-fit on $F^{2}$ & 1.043 & 1.035 & 1.071 \\
\hline$R_{1}[I>2 \sigma(I)]$ (all data) & $0.0356(0.0933)$ & $0.0665(0.1458)$ & $0.0350(0.0880)$ \\
\hline $\mathrm{w} R_{2}[I>2 \sigma(I)]$ (all data) & $0.0421(0.0971)$ & $0.1345(0.1818)$ & $0.0415(0.0909)$ \\
\hline Largest diff. e $\mathrm{A}^{-3}$ & 0.883 and -0.614 & 0.541 and -0.698 & 0.803 and -0.580 \\
\hline
\end{tabular}




\section{S18 Experimental details for the acquisition of X-ray crystallographic data}

Suitable crystals for X-ray diffraction analysis of $1,3,4,6,8$ and 10 were obtained by recrystallization of the purified products. Diffraction data were collected at $150 \mathrm{~K}$ using a Bruker APEX CCD diffractometer with graphite-monochromated MoK $\alpha$ radiation $(\lambda=0.71073 \AA)$. A combination of $\omega$ and $\Phi$ scans was carried out to obtain at least a unique data set. The crystal structures were solved by direct methods, remaining atoms were located from difference Fourier synthesis followed by full-matrix least-squares refinement based on F2 (programs SIR97 and SHELXL-97). ${ }^{[9]}$ Many hydrogen atoms could be found from the Fourier difference analysis. Carbonand oxygen-bound hydrogen atoms were placed at calculated positions and forced to ride on the attached atom. The hydrogen atom contributions were calculated but not refined. All non-hydrogen atoms were refined with anisotropic displacement parameters. The locations of the largest peaks in the final difference Fourier map calculation as well as the magnitude of the residual electron densities were of no chemical significance. Relevant collection and refinement data are summarized in Tables S16. Crystal data and details of data collection and structure refinement for compounds $\mathbf{1}, \mathbf{3}, \mathbf{4 , 6}, \mathbf{8}$ and $\mathbf{1 0}$ (CCDC 862063-862068) can be obtained free of charge from the Cambridge Crystallographic Data Centre via www.ccdc.cam.ac.uk/data_request/cif. 
S19 Full data table for the hydroamination of styrene with $\mathrm{BnNH}_{2}$ catalyzed by $1-10^{[a]}$

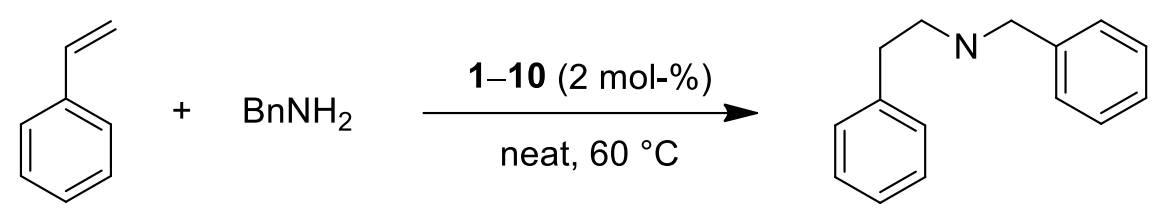

\begin{tabular}{|c|c|c|c|}
\hline Entry & Catalyst & $\mathrm{t}[\mathrm{h}]$ & Conv. $[\%]^{[b)}$ \\
\hline 1 & 1 & 2 & 8 \\
\hline 2 & 2 & 2 & 25 \\
\hline 3 & 3 & 2 & 42 \\
\hline 4 & 1 & 18.5 & 34 \\
\hline 5 & 2 & 18.5 & 71 \\
\hline 6 & 3 & 18.5 & 86 \\
\hline 7 & 4 & 18.5 & 1 \\
\hline 8 & 5 & 18.5 & 6 \\
\hline 9 & 6 & 18.5 & 25 \\
\hline 10 & 7 & 18.5 & 37 \\
\hline 11 & $\left\{\mathrm{~L}^{2}\right\} \mathrm{BaN}\left(\mathrm{SiMe}_{2} \mathrm{H}\right)_{2}$ & 18.5 & 44 \\
\hline 12 & $\left\{\mathrm{~L}^{3}\right\} \mathrm{CaN}\left(\mathrm{SiMe}_{3}\right)_{2}(\mathrm{THF})$ & 2 & 30 \\
\hline 13 & 8 & 2 & 29 \\
\hline 14 & 9 & 2 & 42 \\
\hline 15 & 10 & 2 & 64 \\
\hline
\end{tabular}

[a] Reaction conditions: [styrene] $/\left[\mathrm{BnNH}_{2}\right] /[$ catalyst $]=50: 50: 1$, $10.5 \mu \mathrm{mol}$ of catalyst, no additional solvent, $\mathrm{T}=60^{\circ} \mathrm{C}$. [b]

Determined by ${ }^{1} \mathrm{H}$ NMR spectroscopy. 


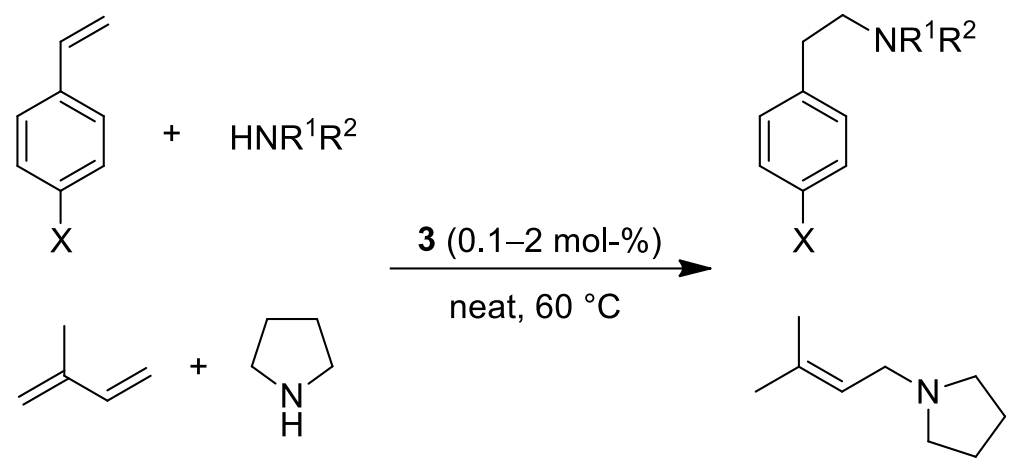

\begin{tabular}{|c|c|c|c|c|c|}
\hline Entry & $X$ & Amine & Alkene/amine/3 & $\mathrm{t}[\mathrm{h}]$ & Conv. $[\%]^{[b)}$ \\
\hline 1 & $\mathrm{H}$ & $i \mathrm{Pr}_{2} \mathrm{NH}_{2}$ & $50: 50: 1$ & 18.5 & 0 \\
\hline 2 & $\mathrm{H}$ & $n \mathrm{HexNH}_{2}$ & $50: 50: 1$ & 2 & 26 \\
\hline 3 & $\mathrm{H}$ & $n \mathrm{HexNH}_{2}$ & $50: 50: 1$ & 18.5 & 55 \\
\hline 4 & $\mathrm{H}$ & $n \mathrm{HexNH}_{2}$ & $50: 50: 1$ & 46 & 70 \\
\hline 5 & $\mathrm{H}$ & $\mathrm{HN}\left(\mathrm{CH}_{2}\right)_{4}$ & $50: 50: 1$ & $<1$ & 99 \\
\hline 6 & $\mathrm{H}$ & $\mathrm{HN}\left(\mathrm{CH}_{2}\right)_{4}$ & 500:500:1 & 2 & 85 \\
\hline 7 & $\mathrm{H}$ & $\mathrm{HN}\left(\mathrm{CH}_{2}\right)_{4}$ & 1000:1000:1 & 2 & 58 \\
\hline 8 & $\mathrm{Me}$ & $\mathrm{BnNH}_{2}$ & $50: 50: 1$ & 2 & 14 \\
\hline 9 & $\mathrm{Me}$ & $\mathrm{BnNH}_{2}$ & $50: 50: 1$ & 18.5 & 41 \\
\hline 10 & $\mathrm{Me}$ & $\mathrm{BnNH}_{2}$ & $50: 50: 1$ & 46 & 60 \\
\hline 11 & $\mathrm{OMe}$ & $\mathrm{BnNH}_{2}$ & $50: 50: 1$ & 2 & 3 \\
\hline 12 & $\mathrm{OMe}$ & $\mathrm{BnNH}_{2}$ & $50: 50: 1$ & 18.5 & 11 \\
\hline 13 & $\mathrm{OMe}$ & $\mathrm{BnNH}_{2}$ & $50: 50: 1$ & 48 & 19 \\
\hline 14 & $\mathrm{OMe}$ & $\mathrm{BnNH}_{2}$ & $50: 50: 1$ & 96 & 35 \\
\hline 15 & $\mathrm{Cl}$ & $\mathrm{BnNH}_{2}$ & $50: 50: 1$ & 2 & 23 \\
\hline 16 & $\mathrm{Cl}$ & $\mathrm{BnNH}_{2}$ & $50: 50: 1$ & 18.5 & 65 \\
\hline 17 & isoprene & $\left(\mathrm{CH}_{2}\right)_{4} \mathrm{NH}$ & 220:50:1 & 1 & $99^{[\mathrm{c}]}$ \\
\hline 18 & isoprene & $\left(\mathrm{CH}_{2}\right)_{4} \mathrm{NH}$ & 2000:1000:1 & 2 & $59^{[\mathrm{c}]}$ \\
\hline
\end{tabular}

[a] Reaction conditions: [alkene]/[amine]/[3] = 50:50:1 unless otherwise specified, $10.5 \mu \mathrm{mol}$ of $\mathbf{3}$, no additional solvent, $\mathrm{T}=60^{\circ} \mathrm{C}$. [b] Determined by ${ }^{1} \mathrm{H}$ NMR spectroscopy. [c] Based on amine conversion. 
S21 Full data table for intermolecular hydrophosphination reactions ${ }^{[a]}$

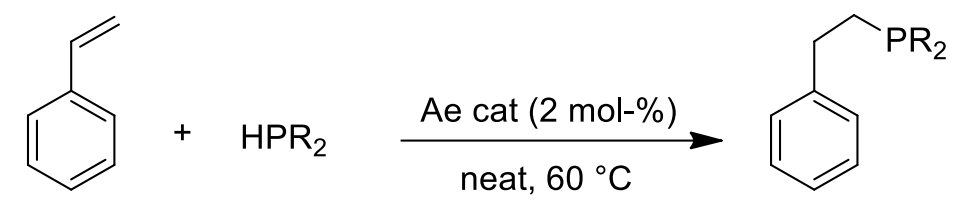

\begin{tabular}{lcccc}
\hline Entry & Catalyst & Phosphine $\mathrm{t}[\mathrm{h}]$ & Conv. [\%] ${ }^{[\mathrm{b}]}$ \\
\hline 1 & $\mathbf{1}$ & $\mathrm{HPCy}_{2}$ & 18.5 & 31 \\
2 & $\mathbf{2}$ & $\mathrm{HPCy}_{2}$ & 18.5 & 41 \\
3 & $\mathbf{3}$ & $\mathrm{HPCy}_{2}$ & 18.5 & 42 \\
4 & $\mathbf{5}$ & $\mathrm{HPCy}_{2}$ & 18.5 & 12 \\
5 & $\mathbf{6}$ & $\mathrm{HPCy}_{2}$ & 18.5 & 26 \\
6 & $\mathbf{7}$ & $\mathrm{HPCy}_{2}$ & 18.5 & 46 \\
7 & $\mathbf{8}$ & $\mathrm{HPCy}_{2}$ & 18.5 & 4 \\
8 & $\mathbf{9}$ & $\mathrm{HPCy}_{2}$ & 18.5 & 9 \\
9 & $\mathbf{1 0}$ & $\mathrm{HPCy}_{2}$ & 18.5 & 18 \\
10 & $\mathbf{1}$ & $\mathrm{HPPh}_{2}$ & 0.25 & 42 \\
11 & $\mathbf{2}$ & $\mathrm{HPPh}_{2}$ & 0.25 & 92 \\
12 & $\mathbf{3}$ & $\mathrm{HPPh}_{2}$ & 0.25 & $>96$ \\
\hline
\end{tabular}

[a] Reaction conditions: [styrene]/[phosphine]/[catalyst] = 50:50:1, $10.5 \mu \mathrm{mol}$ of catalyst, no additional solvent, $\mathrm{T}=$ $60{ }^{\circ} \mathrm{C}$. [b] Determined by ${ }^{1} \mathrm{H}$ NMR spectroscopy. 
S22 Plot of $\ln \left([\text { pyrrolidine }]_{0} /[\text { pyrrolidine }]_{t}\right) v s$. reaction time for the hydroamination of styrene with pyrrolidine catalyzed by 3 at different concentrations of styrene

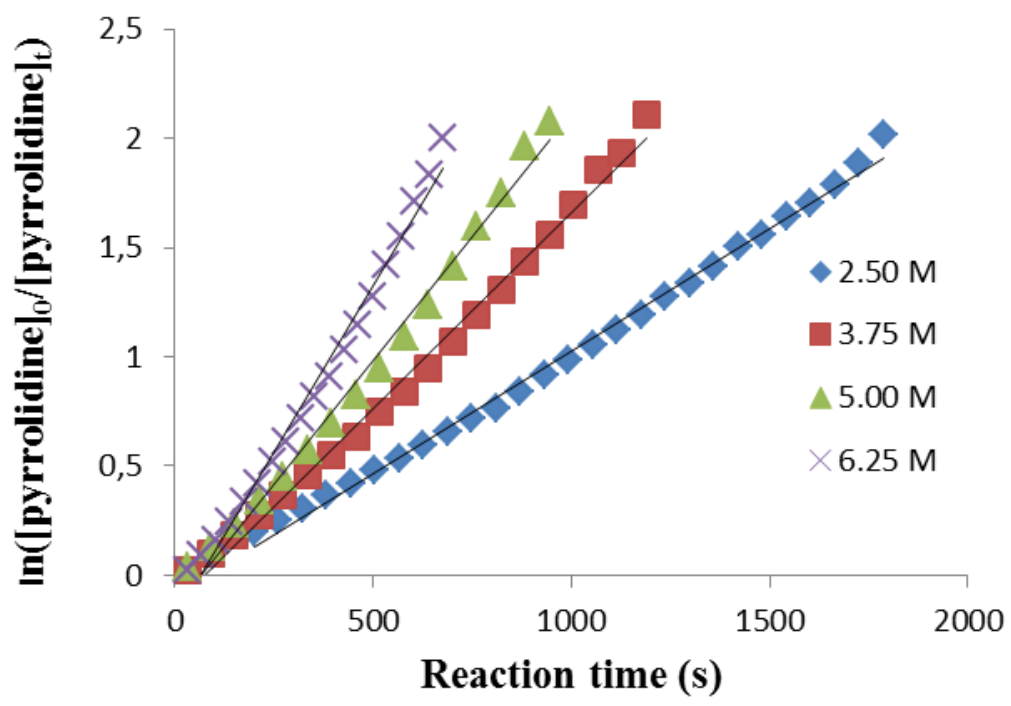

Plot of $\ln \left([\text { pyrrolidine }]_{0} /[\text { pyrrolidine }]_{t}\right) v s$. reaction time (s) for the hydroamination of styrene with pyrrolidine catalyzed by $\left\{\mathrm{L}^{1}\right\} \mathrm{BaN}\left(\mathrm{SiMe}_{3}\right)_{2}(\mathrm{THF})_{2}$ (3) at different concentrations of styrene. Conditions: 3, $10.5 \mu \mathrm{mol}$; pyrrolidine, $13 \mu \mathrm{L}$; pyrrolidine + styrene $+\mathrm{C}_{6} \mathrm{D}_{6}=600 \mu \mathrm{L}, 40{ }^{\circ} \mathrm{C}$.

S23 Plot of $\ln k_{\text {app }} v s . \ln [\text { styrene] }]_{0}$ for the hydroamination of styrene with pyrrolidine catalyzed by 3

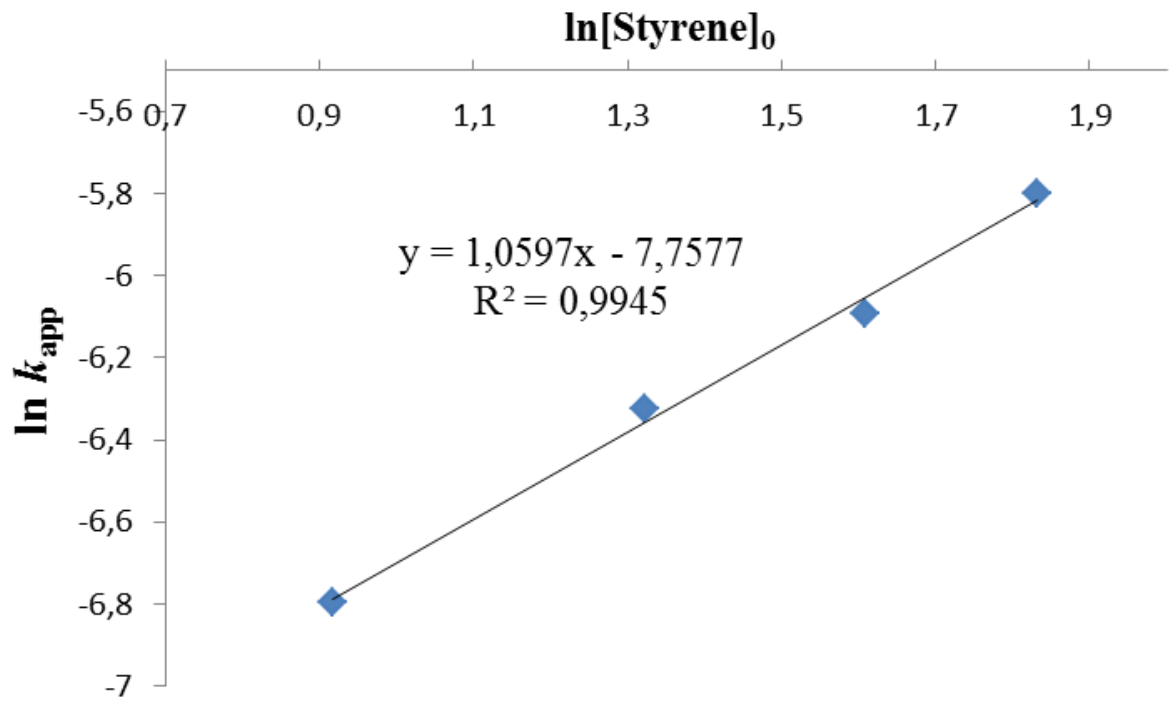

Plot of $\ln k_{\text {app }} \quad v s . \ln \left[\right.$ styrene $_{0}$ for the hydroamination of styrene with pyrrolidine catalyzed by $\left\{\mathrm{L}^{1}\right\} \mathrm{BaN}\left(\mathrm{SiMe}_{3}\right)_{2}(\mathrm{THF})_{2}(\mathbf{3})$. 
S24 Plot of $\ln \left([\text { pyrrolidine }]_{0} /[\text { pyrrolidine }]_{t}\right) v s$. reaction time (s) for the hydroamination of styrene with pyrrolidine catalyzed by 3 at different concentrations of 3

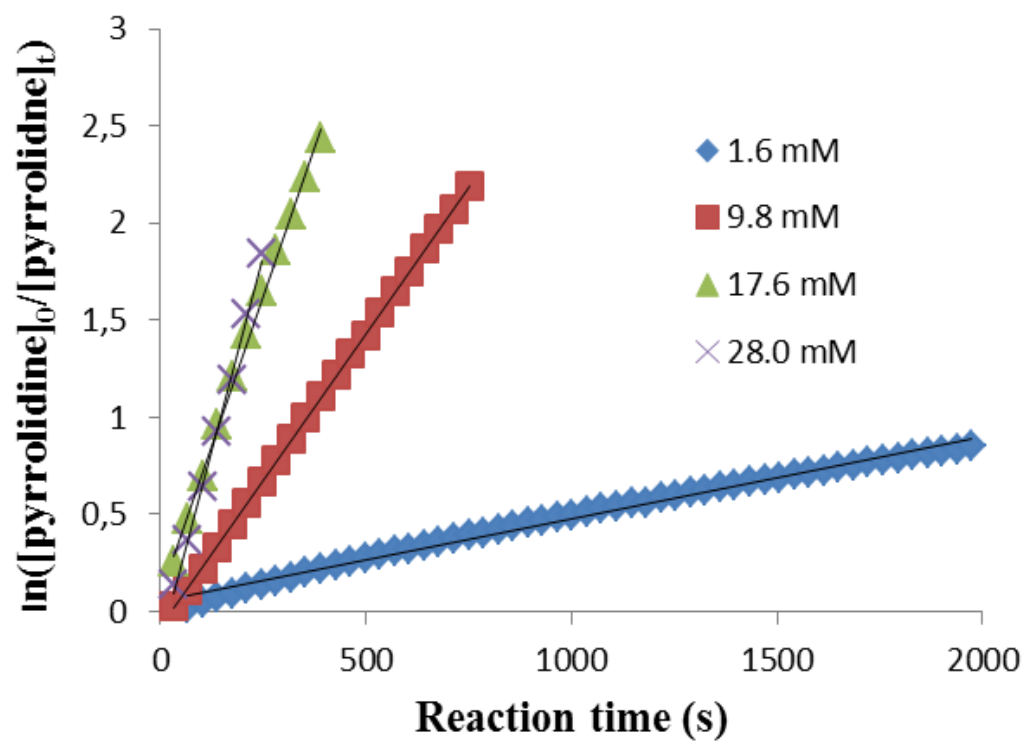

Plot of $\ln \left([\text { pyrrolidine }]_{0} /[\text { pyrrolidine }]_{\mathrm{t}}\right) v s$. reaction time $(\mathrm{s})$ for the hydroamination of styrene with pyrrolidine catalyzed by $\left\{\mathrm{L}^{1}\right\} \mathrm{BaN}\left(\mathrm{SiMe}_{3}\right)_{2}(\mathrm{THF})_{2}(\mathbf{3})$ at different concentration of 3. Reaction conditions: styrene, $173 \mu \mathrm{L}$; pyrrolidine, $13 \mu \mathrm{L}$; pyrrolidine + styrene $+\mathrm{C}_{6} \mathrm{D}_{6}=600 \mu \mathrm{L}, 60{ }^{\circ} \mathrm{C}$.

S25 Plot of $\ln k_{\text {app }} v s . \ln [3]_{0}$ for the hydroamination of styrene with pyrrolidine catalyzed by 3

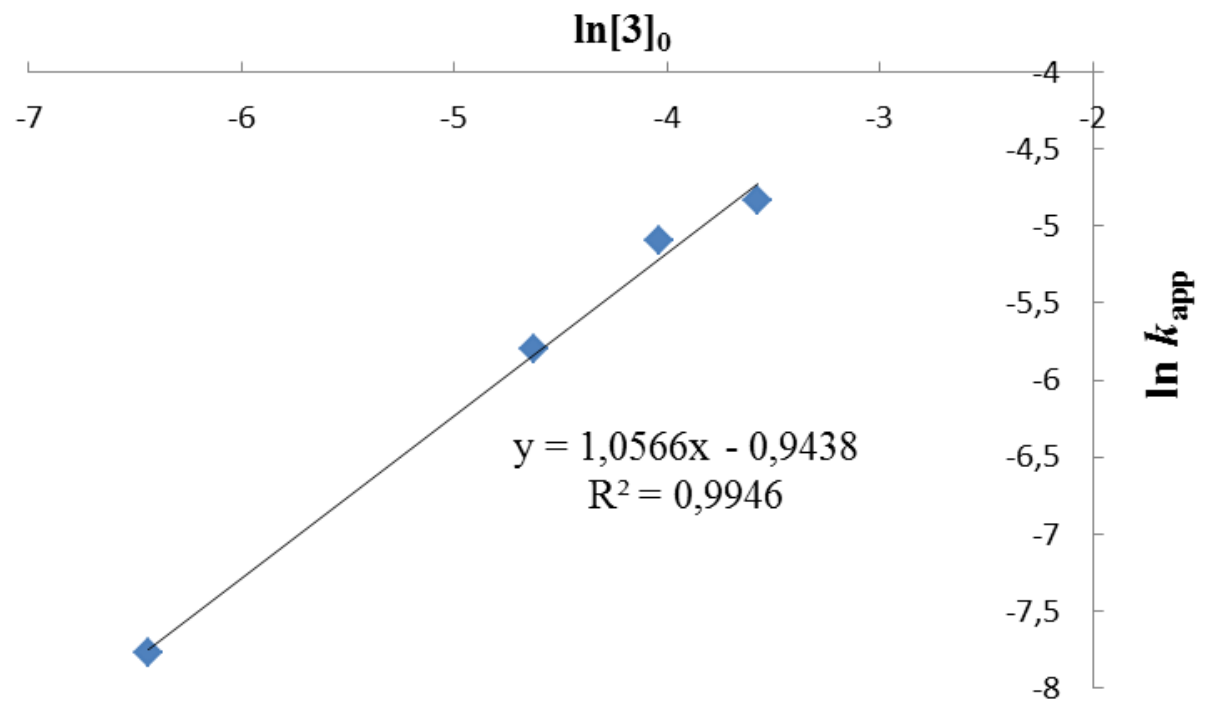

Plot of $\ln k_{\text {app }} v s . \ln [3]_{0}$ for the hydroamination of styrene with pyrrolidine catalyzed by $\left\{\mathrm{L}^{1}\right\} \mathrm{BaN}\left(\mathrm{SiMe}_{3}\right)_{2}(\mathrm{THF})_{2}$ (3). 

pyrrolidine catalyzed by 3 at 333, 323, 313, $298 \mathrm{~K}$

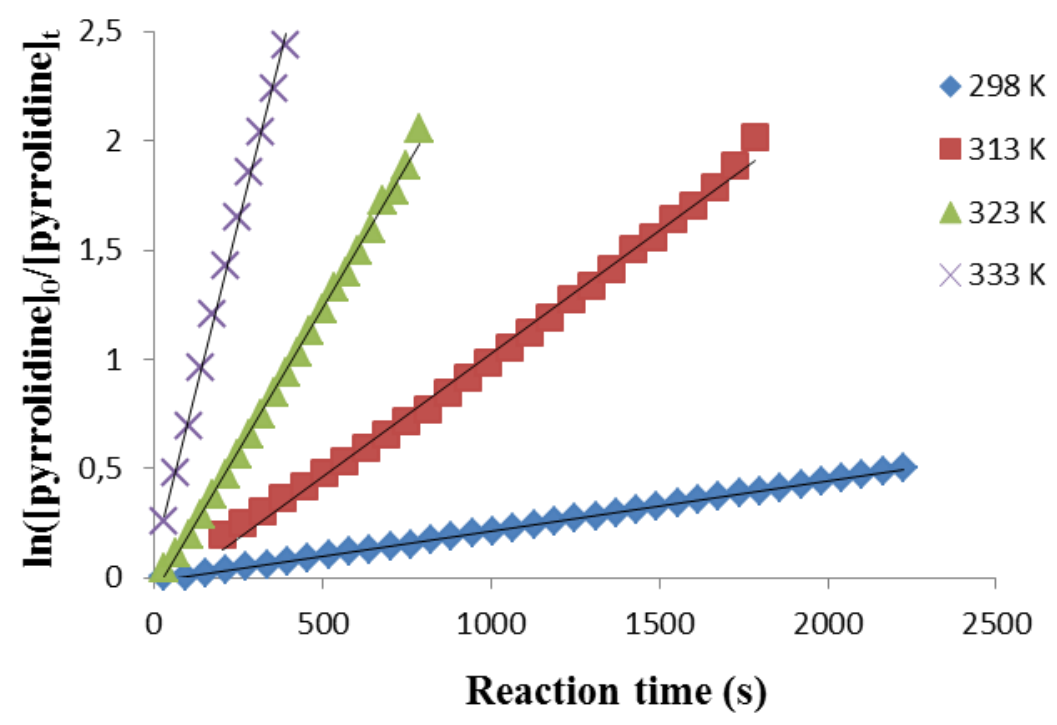

Plot of $\ln \left([\text { pyrrolidine }]_{0} /[\text { pyrrolidine }]_{t}\right) v s$. reaction time $(s)$ for the hydroamination of styrene with pyrrolidine catalyzed by $\left\{\mathrm{L}^{1}\right\} \mathrm{BaN}\left(\mathrm{SiMe}_{3}\right)_{2}(\mathrm{THF})_{2}(\mathbf{3})$ at 333, 323, 313, $298 \mathrm{~K}$. Reaction conditions: 3, $10.5 \mu$ mol; styrene, 173 $\mu \mathrm{L} ;$ pyrrolidine, $13 \mu \mathrm{L}$; pyrrolidine + styrene $+\mathrm{C}_{6} \mathrm{D}_{6}=600 \mu \mathrm{L}$.

S27 Arrhenius plot of $\ln k_{\text {app }} v s .1 / \mathrm{T}\left(\mathrm{K}^{-1}\right)$ for the hydroamination of styrene and pyrrolidine catalyzed by 3

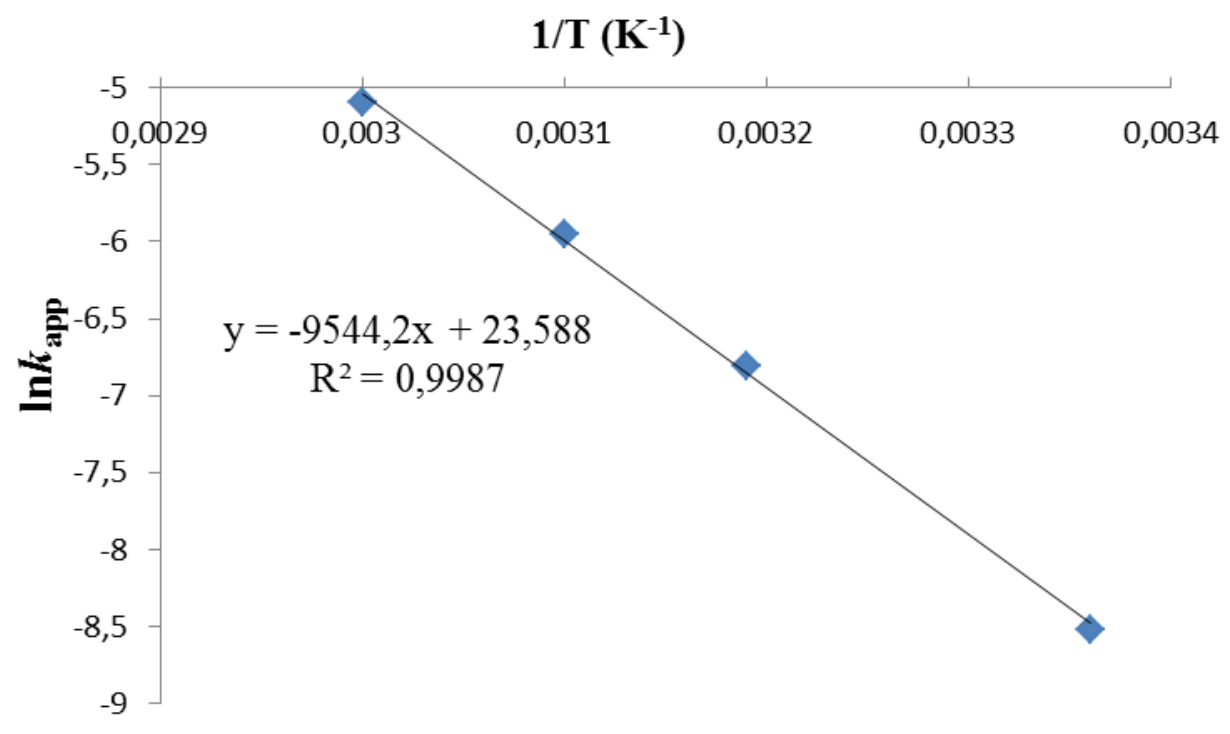

Arrhenius plot of $\ln k_{\text {app }}$ vs. $1 / \mathrm{T}\left(\mathrm{K}^{-1}\right)$ for the hydroamination of styrene and pyrrolidine catalyzed by $\left\{\mathrm{L}^{1}\right\} \mathrm{BaN}\left(\mathrm{SiMe}_{3}\right)_{2}(\mathrm{THF})_{2}(\mathbf{3})$. 
S28 Eyring Plot of $\ln \left(k_{\mathrm{app}} \mathrm{h} / \mathrm{k}_{\mathrm{B}} \mathrm{T}\right)$ vs. 1/T $\left(\mathrm{K}^{-1}\right)$ for the hydroamination of styrene and pyrrolidine catalyzed by 3

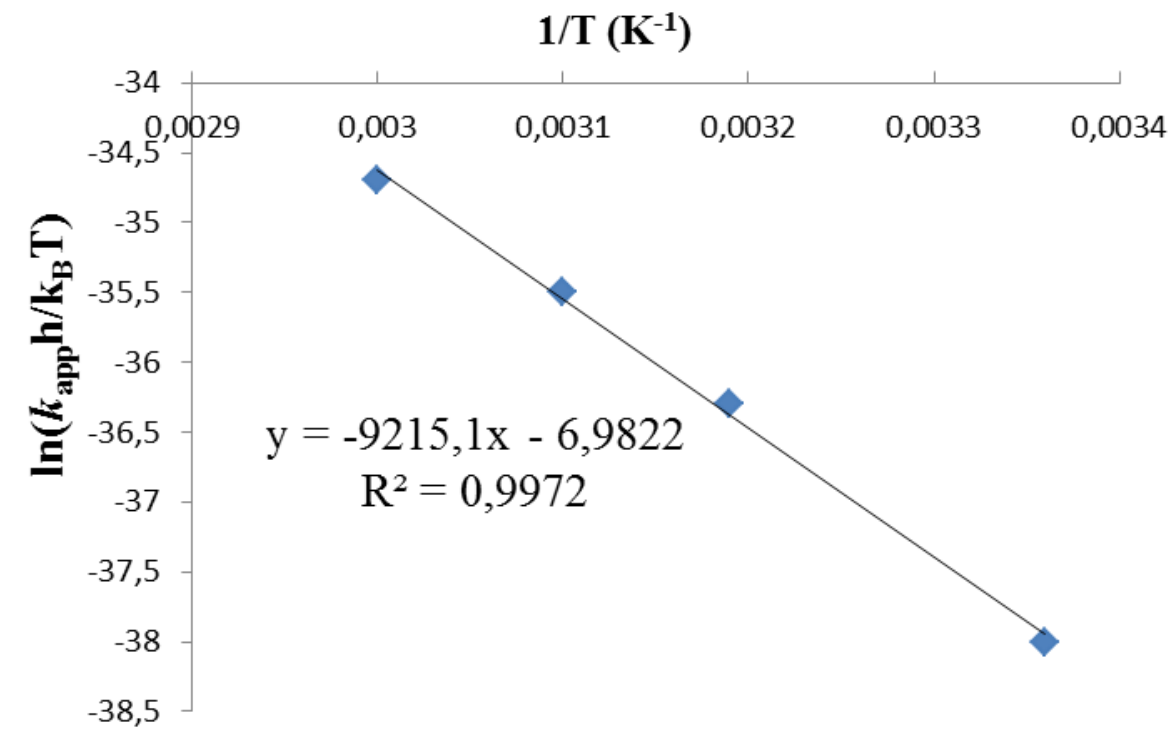

Eyring Plot of $\ln \left(k_{\mathrm{app}} \mathrm{h} / \mathrm{k}_{\mathrm{B}} \mathrm{T}\right)$ vs. $1 / \mathrm{T}\left(\mathrm{K}^{-1}\right)$ for the hydroamination of styrene and pyrrolidine catalyzed by $\left\{\mathrm{L}^{1}\right\} \mathrm{BaN}\left(\mathrm{SiMe}_{3}\right)_{2}(\mathrm{THF})_{2}(\mathbf{3})$. 


\section{S29 Kinetic isotope effect experiments}

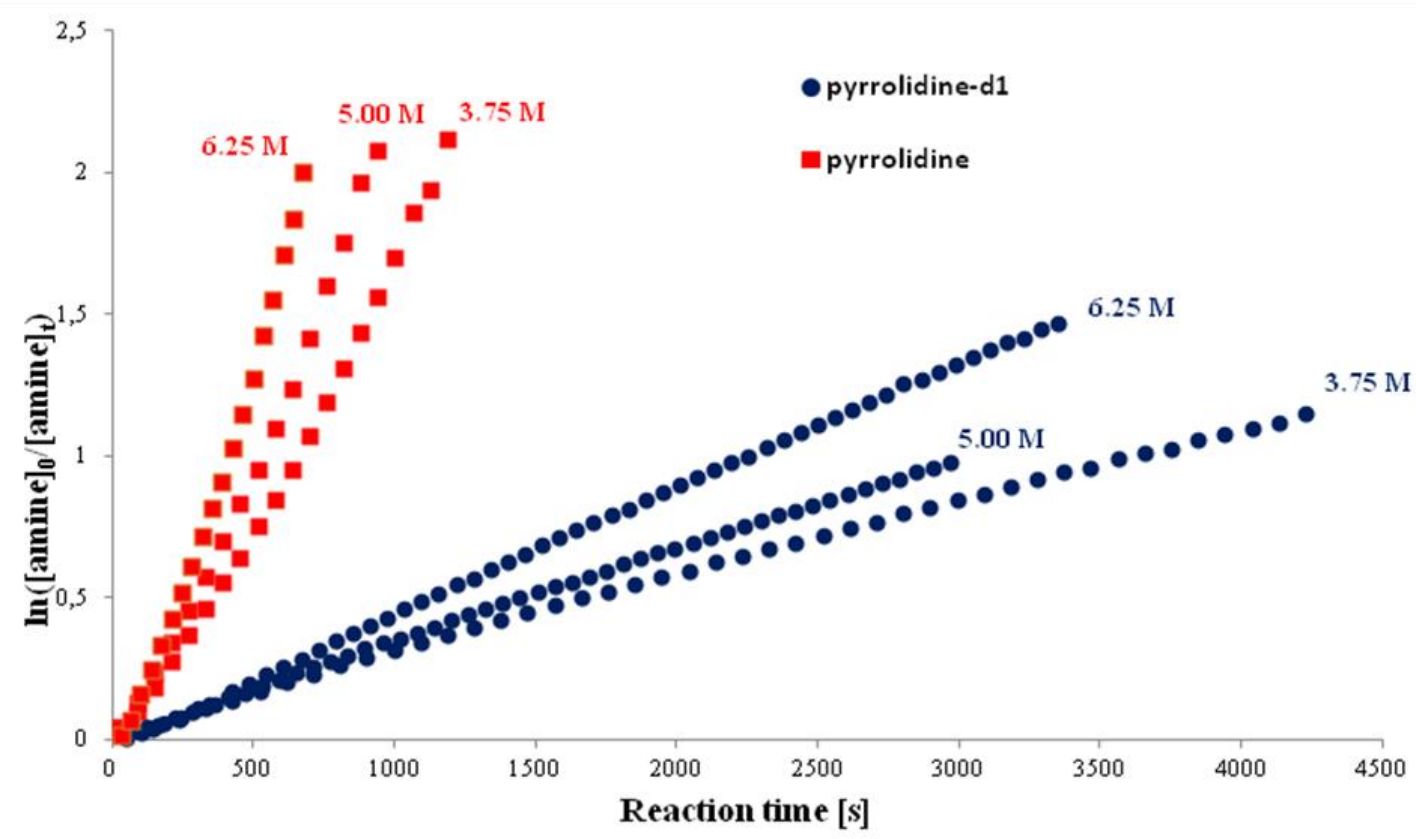

Plot of $\ln \left([\text { amine }]_{0} /[\text { amine }]_{\mathrm{t}}\right)$ vs. reaction time (s) for the hydroamination of styrene with pyrrolidine $(\square)$ and pyrrolidine- $d_{1}(\bullet)$ catalyzed by $\left\{\mathrm{L}^{1}\right\} \mathrm{BaN}\left(\mathrm{SiMe}_{3}\right)_{2}(\mathrm{THF})_{2}(\mathbf{3})$ at different concentrations of styrene $(3.75,5.00$ and 6.25 M). Conditions: 3, $10.5 \mu \mathrm{mol}$; amine, $13 \mu \mathrm{L}$; amine + styrene $+\mathrm{C}_{6} \mathrm{D}_{6}=600 \mu \mathrm{L}, 40{ }^{\circ} \mathrm{C}$.

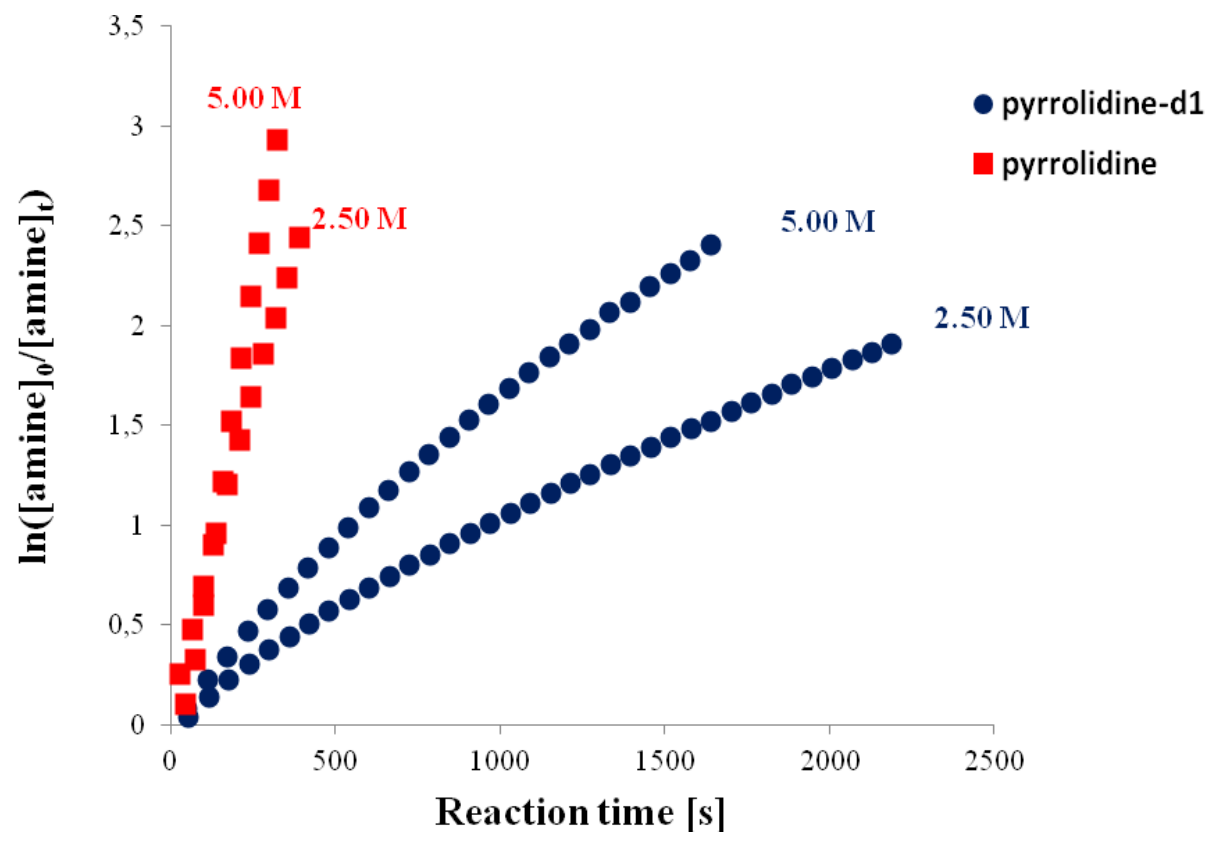

Plot of $\ln \left([\text { amine }]_{0} /[\text { amine }]_{t}\right)$ vs. reaction time (s) for the hydroamination of styrene with pyrrolidine $(\square)$ and pyrrolidine- $d_{1}(\bullet)$ catalyzed by $\left\{\mathrm{L}^{1}\right\} \mathrm{BaN}\left(\mathrm{SiMe}_{3}\right)_{2}(\mathrm{THF})_{2}(3)$ at different concentrations of styrene $(5.00$ and 2.50 M). Conditions: 3, $10.5 \mu \mathrm{mol}$; amine, $13 \mu \mathrm{L}$; amine + styrene $+\mathrm{C}_{6} \mathrm{D}_{6}=600 \mu \mathrm{L}, 60^{\circ} \mathrm{C}$. 


\section{S30 References}

[1] L. García-Río, J. R. Leis, J. A. Moreira, D. Serantes, Eur. J. Org. Chem., 2004, 614-622.

[2] Y. Sarazin, D. Roşca, V. Poirier, T. Roisnel, A. Silvestru, L. Maron, J.-F. Carpentier, Organometallics, 2010, 29, 6569-6577.

[3] a) J. M. Boncella, C. J. Coston, J. K. Cammack, Polyhedron, 1991 10, 769-770; b) Y. Sarazin, R. H. Howard, D. L. Hughes, S. M. Humphrey, M. Bochmann, Dalton Trans., 2006, 340-350.

[4] Y. Sarazin, B. Liu, T. Roisnel, L. Maron, J.-F. Carpentier, J. Am. Chem. Soc., 2011, 133, 9069-9087.

[5] B. Liu, T. Roisnel, Y. Sarazin, Inorg. Chim. Acta, 2012, 380, 2-13.

[6] P. G. Hayes, G. C. Welch, D. J. H. Emslie, C. L. Noack, W. E. Piers, M. Parvez, Organometallics, 2003, $22,1577-1579$.

[7] S. Groysman, E. Sergeeva, I. Goldberg, M. Kol, Inorg. Chem., 2005, 44, 8188-8190

[8] J. E. Parks, R. H. Holm, Inorg. Chem. 1968, 7, 1408-1416.

[9] a) G. M. Sheldrick, SHELXS-97, Program for the Determination of Crystal Structures; University of Goettingen: Germany, 1997; b) G. M. Sheldrick, SHELXL-97, Program for the Refinement of Crystal Structures; University of Goettingen: Germany, 1997. 